\title{
A CONTINUOUS INTERPOLATION BETWEEN CONSERVATIVE AND DISSIPATIVE SOLUTIONS FOR THE TWO-COMPONENT CAMASSA-HOLM SYSTEM
}

\author{
KATRIN GRUNERT ${ }^{1}$, HELGE HOLDEN $^{1,2}$ and XAVIER RAYNAUD ${ }^{1,3}$ \\ ${ }^{1}$ Department of Mathematical Sciences, Norwegian University of Science and Technology, \\ NO-7491 Trondheim, Norway; \\ email: katring@math.ntnu.no \\ ${ }^{2}$ Centre of Mathematics for Applications, University of Oslo, NO-0316 Oslo, Norway; \\ email: holden@math.ntnu.no \\ ${ }^{3}$ SINTEF ICT, Applied Mathematics, P.O. Box 124, NO-0314 Oslo, Norway; \\ email: raynaud@math.ntnu.no
}

Received 18 March 2014; accepted 27 November 2014

\begin{abstract}
We introduce a novel solution concept, denoted $\alpha$-dissipative solutions, that provides a continuous interpolation between conservative and dissipative solutions of the Cauchy problem for the twocomponent Camassa-Holm system on the line with vanishing asymptotics. All the $\alpha$-dissipative solutions are global weak solutions of the same equation in Eulerian coordinates, yet they exhibit rather distinct behavior at wave breaking. The solutions are constructed after a transformation into Lagrangian variables, where the solution is carefully modified at wave breaking.
\end{abstract}

2010 Mathematics Subject Classification: 35Q53, 35B35 (primary); 35Q20 (secondary)

\section{Introduction}

We consider the Cauchy problem for the two-component Camassa-Holm $(2 \mathrm{CH})$ system given by

$$
\begin{gathered}
u_{t}-u_{t x x}+\kappa u_{x}+3 u u_{x}-2 u_{x} u_{x x}-u u_{x x x}+\eta \rho \rho_{x}=0, \\
\rho_{t}+(u \rho)_{x}=0,
\end{gathered}
$$

with initial data $\left.u\right|_{t=0}=u_{0}$ and $\left.\rho\right|_{t=0}=\rho_{0}$. Here, $\kappa \in \mathbb{R}$ and $\eta \in(0, \infty)$ are given parameters. We are interested in global weak solutions for general initial data

(C) The Author(s) 2015. This is an Open Access article, distributed under the terms of the Creative Commons Attribution licence (http://creativecommons.org/licenses/by/3.0/), which permits unrestricted re-use, distribution, and reproduction in any medium, provided the original work is properly cited. 


$$
u_{0} \in H^{1}(\mathbb{R}) \quad \text { and } \quad \rho_{0} \in L^{2}(\mathbb{R}) .
$$

The $2 \mathrm{CH}$ system was introduced by Olver and Rosenau [35, Equation (43)] (see also $[2,8,32]$ ), and derived in the context of water waves by Constantin and Ivanov [11]. In this paper, the question of wave breaking is also analyzed. The scalar $\mathrm{CH}$ equation, which corresponds to the case where $\rho(t, x)=\rho_{0}(x)=0$, was introduced by Camassa and Holm in the fundamental paper [7], and its analysis has been pervasive. Other generalizations of the Camassa-Holm equation exist; see, for example, $[8,9,13,21,33]$.

The $2 \mathrm{CH}$ system experiences wave breaking in the sense that the spatial derivative of $u$ becomes unbounded while keeping its $H^{1}(\mathbb{R})$ norm finite. This gives rise to a dichotomy between so-called conservative and dissipative solutions, which complicates the issue of well posedness of the Cauchy problem. This issue has been studied extensively [15, 17, 18, 34, 37]. Analysis of blow-up and existence of global solutions for the $2 \mathrm{CH}$ system can be found in, for example, $[19,22-25,30,31]$.

In this article, we introduce a novel class of solutions parameterized by $\alpha \in[0$, 1]. The parameter $\alpha$ determines the amount of dissipation for the corresponding class of solutions. If $\alpha=0$, there is no dissipation, and we obtain the conservative solutions, meaning that, when a collision (that is, wave breaking) occurs, the energy contained in the collision is entirely redistributed in the system after the collision. If $\alpha=1$, we obtain the (fully) dissipative solutions, where all the energy contained in a collision vanishes from the system. The intermediate values of $\alpha$ give the fraction of the energy contained in the collision which is dissipated. The remaining energy is given back after the collision.

For simplicity, in this introduction, we consider first the $\mathrm{CH}$ equation with $\kappa=$ 0 . However, in the text proper, we analyze the full $2 \mathrm{CH}$ system. Dissipation occurs when the solution blows up. The problem of blow-up can be studied explicitly in the case of multipeakon solutions, but since this example is well known, we refer to, for example, [26], where this is well described, rather than presenting the details here. The upshot of the analysis is that the solution $u$ has to be augmented by an additional variable in the form of a measure, denoted $\mu$, that describes the energy. For $u_{0} \in H^{1}(\mathbb{R})$, we let $\mu=u_{x}^{2} d x$. For smooth solutions to the $\mathrm{CH}$ equation, the following conservation law for the energy holds:

$$
\left(u^{2}+u_{x}^{2}\right)_{t}+\left(u\left(u^{2}+u_{x}^{2}\right)\right)_{x}=\left(u^{3}-2 P u\right)_{x},
$$

which implies that the total energy, that is, the $H^{1}(\mathbb{R})$ norm of $u$, is preserved. Here, $P$ is an integrated term which is defined below; see (4). When blow-up occurs, the energy density $\left(u^{2}+u_{x}^{2}\right) d x$ becomes singular; that is, it becomes a measure containing a singular part. This measure has to be augmented to the solution $u$ in order to be able to define the continuation after blow-up. 
The proper way to continue the solution after blow-up is to rewrite the equation in terms of new variables, denoted Lagrangian variables, where the $\mathrm{CH}$ equation appears as a system of ordinary differential equations taking values in a Banach space in such a way that the blow-up in the original Eulerian variables (1) evaporates $[4,5,27,29]$. In the present literature, the analysis has been distinct for the two classes of solutions. Our new solution concept governed by the parameter $\alpha$ allows for a continuous interpolation between the conservative and dissipative solutions. At the same time it allows a uniform treatment of all cases. We denote these solutions as $\alpha$-dissipative solutions.

Let us describe more precisely the construction of the $\alpha$-dissipative solutions. After applying the inverse Helmholtz operator $\left(1-\partial_{x x}\right)^{-1}$, the $\mathrm{CH}$ equation can be rewritten as

$$
u_{t}+u u_{x}+P_{x}=0, \quad P-P_{x x}=u^{2}+\frac{1}{2} u_{x}^{2} .
$$

The pattern of blow-up is known [10]: the solution remains continuous while the derivative $u_{x}$ tends to minus infinity at the blow-up point. For this reason, the blow-up for the $\mathrm{CH}$ equation is often characterized as wave breaking, and we will use this term extensively in this paper. Wave breaking occurs precisely when the characteristics, $y=y(t, \xi)$, given by

$$
y_{t}(t, \xi)=u(t, y(t, \xi))
$$

have a critical point; that is, $y_{\xi}(t, \xi)=0$. For a given 'particle', labeled by $\xi$, the characteristic $y(t, \xi)$ denotes the trajectory of $\xi$, and

$$
\tau_{1}(\xi)= \begin{cases}\sup \left\{t \in \mathbb{R}_{+} \mid y_{\xi}\left(t^{\prime}, \xi\right)>0 \text { for all } 0<t^{\prime}<t\right\} & \text { if }\{\cdots\} \neq \emptyset \\ \infty & \text { otherwise }\end{cases}
$$

denotes the time of the first wave breaking for $\xi$. For dissipative solutions, we would set $y_{\xi}(t, \xi)=0$ for $t>\tau_{1}(\xi)$, while for conservative solutions we would continue to use (5). Typically, in a collision taking place at time $t_{c}$, the trajectories of different particles meet, say $y\left(t_{c}, \xi_{1}\right)=y\left(t_{c}, \xi\right)=y\left(t_{c}, \xi_{2}\right)$ for $\xi \in\left[\xi_{1}, \xi_{2}\right]$. In the dissipative case, the particles remain together. The energy, which in the case of conservative solutions sends the collided particles apart, is entirely dissipated in the dissipative case. To keep track of the part of the energy that accumulates at collision points, we introduce the function

$$
h(t, \xi)=u_{x}^{2}(t, y(t, \xi)) y_{\xi}(t, \xi) .
$$

The time evolution of $h$ is given by

$$
h_{t}(t, \xi)=2\left(U^{2}(t, \xi)-P(t, \xi)\right) U_{\xi}(t, \xi),
$$


where the function

$$
U(t, \xi)=u(t, y(t, \xi))
$$

denotes the Lagrangian velocity. We write the $\mathrm{CH}$ equation as a system of ordinary differential equations in Lagrangian coordinates:

$$
\begin{gathered}
y_{t}=U, \quad U_{t}=-Q, \quad h_{t}=2\left(U^{2}-P\right) U_{\xi}, \\
y_{t, \xi}=U_{\xi}, \quad U_{t, \xi}=\frac{1}{2} h+\left(U^{2}-P\right) y_{\xi},
\end{gathered}
$$

where $P$ and $Q$ are integrated terms, enjoying higher regularity, given by (24) and (21), respectively. The control on the level of dissipation, which depends on $\alpha$, is determined by the Lagrangian variables at the times of collision. At collision time $\tau_{1}(\xi)$, for the particle $\xi$, we decompose $h$ into two parts:

$$
h\left(\tau_{1}(\xi), \xi\right)=\alpha h\left(\tau_{1}(\xi), \xi\right)+(1-\alpha) h\left(\tau_{1}(\xi), \xi\right) .
$$

For $\alpha$-dissipative solutions, the first part is dissipated, while the second is redistributed to the system. We introduce $\bar{h}$, which denotes the effective part of the energy, that is, the part which effectively amounts for the energy that is left after a collision. Before the first collision, $h$ and $\bar{h}$ coincide, but at collision time, $\bar{h}$ is discontinuous, and we set

$$
\bar{h}\left(\tau_{1}(\xi), \xi\right)=(1-\alpha) \lim _{t \uparrow \tau_{1}(\xi)} \bar{h}(t, \xi),
$$

while $h$ remains continuous in time. In fact, it should be enough only to consider $\bar{h}$ instead of $h$; however, the variable $h$, because of its time continuity property, is so useful in the proofs that we keep it as one of the variables for the governing equations. The same particle may experience additional collisions later. Thus, we construct the sequence

$$
0<\tau_{1}(\xi)<\tau_{2}(\xi)<\cdots<\tau_{j}(\xi)<\cdots
$$

of collision times. For a given $\xi$, the sequence $\tau_{i}(\xi)$ does not accumulate, and there exists a lower bound for the time separating two collisions; see Corollary 19. At each $\tau_{j}(\xi)$, we reset $\bar{h}$; that is,

$$
\bar{h}\left(\tau_{j}(\xi), \xi\right)=(1-\alpha) \lim _{t \uparrow \tau_{j}(\xi)} \bar{h}(t, \xi) .
$$

The equations in Lagrangian coordinates we will consider are given by

$$
\begin{gathered}
y_{t}=U, \\
U_{t}=-Q,
\end{gathered}
$$




$$
\begin{gathered}
y_{t, \xi}=U_{\xi}, \\
U_{t, \xi}=\frac{1}{2} \bar{h}+\left(U^{2}-P\right) y_{\xi}, \\
h_{t}=2\left(U^{2}-P\right) U_{\xi},
\end{gathered}
$$

where $P$ and $Q$ are given by (33) and (34), respectively. The initial characteristics are given by $y(\xi)=\sup \{y \mid \mu((-\infty, y))+y<\xi\}$. Note that, since $\bar{h}$ is discontinuous, the system of ordinary differential equations (10) is discontinuous.

Now we want to obtain a global solution of the system (10), properly formulated. We consider the vector $\Theta=\left(\zeta, U, \zeta_{\xi}, U_{\xi}, \bar{h}, h\right) \in L^{\infty}(\mathbb{R}) \times E^{5}$, where $E=L^{2}(\mathbb{R}) \cap L^{\infty}(\mathbb{R})$, and, for technical reasons, we prefer to work with $\zeta=y-\mathrm{Id}$. In order to obtain a global solution that respects the intrinsic structure of the system, we have to restrict the initial data appropriately, and we only consider initial data in the set $\mathcal{G}$ given by Definition 3. Short-time existence is proved by an iteration argument (see Theorem 15), and existence of a global solution in $\mathcal{G}$ is proved in Theorem 17.

The next task is then to return to Eulerian coordinates, where the solution $(u(t)$, $\mu(t))$ for each positive time $t$ satisfies $u(t) \in H^{1}(\mathbb{R})$, as well as being a weak global solution of (4), and $\mu(t)$ is a nonnegative Radon measure such that $\mu_{\mathrm{ac}}(t)=$ $u_{x}^{2}(t, \cdot) d x$. When $u$ is a smooth solution, $\mu=\mu_{\mathrm{ac}}$, but, at a blow-up time $t_{c}$, the singular part of $\mu$, which we denote $\mu_{\mathrm{s}}$, accounts for the singular part of the energy, as we have

$$
\lim _{t \uparrow t_{c}}\left(\left(u^{2}(t, x)+u_{x}^{2}(t, x)\right) d x\right)=\mu_{\mathrm{s}}\left(t_{c}\right)+\left(u^{2}\left(t_{c}, x\right)+u_{x}^{2}\left(t_{c}, x\right)\right) d x .
$$

The next problem is that of relabeling; there are several distinct Lagrangian solutions corresponding to one and the same solution in Eulerian variables, similar to the fact that there are several distinct parameterizations of one and the same curve. We identify the precise set $G$ of relabeling functions (see Definition 5), and we show that the flow respects the relabeling (see Theorem 24). The return to Eulerian variables is contained in Definition 8, where we define

$$
\begin{gathered}
u(x)=U(\xi) \text { for any } \xi \text { such that } x=y(\xi), \\
\mu=y_{\#}(\bar{h}(\xi) d \xi) .
\end{gathered}
$$

Here we used the convention to denote the push-forward of the measure $d \sigma$ by the function $y$ as $v=y_{\#}(d \sigma)$, where $v(A)=\sigma\left(y^{-1}(A)\right)$. Finally, we show that the solution is a global weak solution of the $\mathrm{CH}$ equation, and that we have (see Theorem 26)

$$
\left(u^{2}+\mu\right)_{t}+\left(u\left(u^{2}+\mu\right)\right)_{x} \leqslant\left(u^{3}-2 P u\right)_{x}
$$

in the sense of distributions. 
Until now, we have focused on the $\mathrm{CH}$ equation, that is, the case where $\rho(t$, $x)=\rho_{0}(x)=0$, which implies that $(1 a)$ and $(1 b)$ decouple. For the $2 \mathrm{CH}$ system in the general case, when $\rho_{0} \neq 0$, we observe the same regularization properties as in the conservative case presented in [15], namely that, if $\rho_{0}(x)>0$ for all $x$, then the solution retains the same level of regularity as the one it has initially, no collision occurs, and

$$
E(t)=E(0)
$$

for all times $t$, where $E(t)=\sqrt{\|u(t, \cdot)\|_{H^{1}}^{2}+\|\rho(t, \cdot)\|_{L^{2}}^{2}}$. For general initial data, if $\alpha=0$, the identity (12) holds only for almost every time $t$, while, if $\alpha>0$, the function $E(t)$ is then nonincreasing almost everywhere, that is,

$$
E(t) \leqslant E\left(t^{\prime}\right)
$$

for $t>t^{\prime}$, where $t$ and $t^{\prime}$ belong to a given set of full measure; see Theorems 26 and 27.

Finally, we present in Section 5 detailed calculations for the explicit example of a peakon-antipeakon solution. Here, one can see the interplay between Eulerian and Lagrangian variables, and the role and use of relabeling, as well as an explicit description of the behavior at wave breaking.

\section{Lagrangian setting}

We consider the Cauchy problem for the two-component Camassa-Holm system with arbitrary $\kappa \in \mathbb{R}$ and $\eta \in(0, \infty)$, given by

$$
\begin{gathered}
u_{t}-u_{t x x}+\kappa u_{x}+3 u u_{x}-2 u_{x} u_{x x}-u u_{x x x}+\eta \rho \rho_{x}=0, \\
\rho_{t}+(u \rho)_{x}=0,
\end{gathered}
$$

with initial data $\left.u\right|_{t=0}=u_{0}$ and $\left.\rho\right|_{t=0}=\rho_{0}$, such that $u \in H^{1}(\mathbb{R})$ and $\rho \in L^{2}(\mathbb{R})$. A close look reveals that, if $(u(t, x), \rho(t, x))$ is a solution of the two-component Camassa-Holm system (14), then we easily find that

$$
v(t, x)=u(t, x), \quad \text { and } \quad \tau(t, x)=\sqrt{\eta} \rho(t, x),
$$

solves the two-component Camassa-Holm system with $\eta=1$. Therefore, without loss of generality, we assume in what follows that $\eta=1$. Our analysis does not extend to the case with $\eta$ negative. For results in that case, see, for example, [12]. In addition, we only consider the case $\kappa=0$, as one can make the same conclusions for $\kappa \neq 0$ with slight modifications. The general case with $\kappa \in \mathbb{R}$, which is related to the case where the solution $u, \rho$ has nonvanishing asymptotics, is treated in $[14,15,18]$.

In the remainder of this section, we will introduce the set of Lagrangian coordinates we want to work with, and the corresponding Banach space. 
2.1. Reformulation of the $\mathbf{2 C H}$ system in Lagrangian coordinates. The $2 \mathrm{CH}$ system with $\kappa=0$ can be rewritten as the following system in Eulerian coordinates:

$$
\begin{gathered}
u_{t}+u u_{x}+P_{x}=0, \\
\rho_{t}+(u \rho)_{x}=0, \\
P-P_{x x}=u^{2}+\frac{1}{2} u_{x}^{2}+\frac{1}{2} \rho^{2},
\end{gathered}
$$

where $P$ and $P_{x}$ are given by

$$
P(t, x)=\frac{1}{2} \int_{\mathbb{R}} e^{-|x-z|}\left(u^{2}+\frac{1}{2} u_{x}^{2}+\frac{1}{2} \rho^{2}\right)(t, z) d z,
$$

and

$$
P_{x}(t, x)=-\frac{1}{2} \int_{\mathbb{R}} \operatorname{sgn}(x-z) e^{-|x-z|}\left(u^{2}+\frac{1}{2} u_{x}^{2}+\frac{1}{2} \rho^{2}\right)(t, z) d z .
$$

(For $\kappa$ nonzero, (16c) is simply replaced by $P-P_{x x}=u^{2}+\kappa u+\frac{1}{2} u_{x}^{2}+$ $\frac{1}{2} \rho^{2}$.) In order to reformulate system (16) in Lagrangian variables, we define the characteristics $y(t, \xi)$ as the solution of

$$
y_{t}(t, \xi)=u(t, y(t, \xi))
$$

for a given $y(0, \xi)$. The Lagrangian velocity is given by $U(t, \xi)=u(t, y(t, \xi))$, and we find using (16a) that

$$
U_{t}(t, \xi)=-Q(t, \xi),
$$

where $Q(t, \xi)=P_{x}(t, y(t, \xi))$ is given by

$$
Q(t, \xi)=-\frac{1}{4} \int_{\mathbb{R}} \operatorname{sgn}(\xi-\eta) e^{-|y(t, \xi)-y(t, \eta)|}\left(2 U^{2} y_{\xi}+h\right)(t, \eta) d \eta,
$$

where we have introduced $h=\left(u_{x}^{2}+\rho^{2}\right) \circ y y_{\xi}$, or

$$
h(t, \xi)=\left(u_{x}^{2}(t, y(t, \xi))+\rho^{2}(t, y(t, \xi))\right) y_{\xi}(t, \xi) .
$$

The time evolution of $h(t, \xi)$ is given by

$$
h_{t}(t, \xi)=2\left(U^{2}(t, \xi)-P(t, \xi)\right) U_{\xi}(t, \xi),
$$

where $P(t, \xi)=P(t, y(t, \xi))$ is given by

$$
P(t, \xi)=\frac{1}{4} \int_{\mathbb{R}} e^{-|y(t, \xi)-y(t, \eta)|}\left(2 U^{2} y_{\xi}+h\right)(t, \eta) d \eta .
$$


Last, but not least, the Lagrangian density

$$
r(t, \xi)=\rho \circ y y_{\xi}(t, \xi)=\rho(t, y(t, \xi)) y_{\xi}(t, \xi)
$$

is preserved with respect to time; that is,

$$
r_{t}=0
$$

according to $(16 b)$.

We have formally reformulated the $2 \mathrm{CH}$ system (16) in Eulerian coordinates as the following system of ordinary differential equations in Lagrangian variables:

$$
\begin{gathered}
y_{t}=U, \\
U_{t}=-Q, \\
y_{t, \xi}=U_{\xi}, \\
U_{t, \xi}=\frac{1}{2} h+\left(U^{2}-P\right) y_{\xi}, \\
h_{t}=2\left(U^{2}-P\right) U_{\xi}, \\
r_{t}=0,
\end{gathered}
$$

where $P$ and $Q$ are given by (24) and (21), respectively.

2.2. The new solution concept: $\boldsymbol{\alpha}$-dissipative solutions. Wave breaking for the $2 \mathrm{CH}$ system means that $u_{x}$ becomes pointwise unbounded from below, which is equivalent, in this case, to saying that $y_{\xi}$ becomes zero. Let therefore $\tau_{1}(\xi)$ denote the first time when $y_{\xi}(t, \xi)$ vanishes at the point $\xi$; that is,

$$
\tau_{1}(\xi)=\sup \left\{t \in \mathbb{R}_{+} \mid y_{\xi}\left(t^{\prime}, \xi\right)>0 \text { for all } 0<t^{\prime}<t\right\}
$$

if there exists some $t>0$ such that $y_{\xi}\left(t^{\prime}, \xi\right)>0$ for all $t^{\prime} \in(0, t)$ and $y_{\xi}(t$, $\xi)=0$. Otherwise, we set $\tau_{1}(\xi)=\infty$. For conservative solutions, we would continue $y_{\xi}(t, \xi)$ past wave breaking according to the definition (19), while for dissipative solutions one sets $y(t, \xi)$ constant in $\xi$ (not in time), that is, $y_{\xi}(t$, $\xi)=0$, after wave breaking. It turns out that the proper way to interpolate between the two solutions is by using the variable $h(t, \xi)$ given by (22). For $\alpha \in[0,1]$, we extend the solution past wave breaking by instantaneously reducing the function $h(t, \xi)$ by a factor $(1-\alpha)$ at wave breaking. More precisely, we introduce an extra energy variable, $\bar{h}$, which corresponds to the energy which is actually contained in the system and which coincides with $h$ until wave breaking occurs for the first time. At each collision, $\bar{h}$ is going to be discontinuous in time (for $\alpha>0$ ) as we set

$$
\bar{h}\left(\tau_{1}(\xi), \xi\right)=(1-\alpha) \bar{h}\left(\tau_{1}(\xi)-0, \xi\right) .
$$


Here we use the notation $\Phi(x \pm 0)=\lim _{\varepsilon \downarrow 0} \Phi(x \pm \varepsilon)$. The energy variable $h$ remains continuous in time, as we set

$$
h\left(\tau_{1}(\xi), \xi\right)=h\left(\tau_{1}(\xi)-0, \xi\right) .
$$

We define by induction the times $\tau_{n}(\xi)$, for $\xi$ fixed, where collisions occur. Let

$$
\tau_{n}(\xi)=\sup \left\{t \in\left(\tau_{n-1}(\xi), \infty\right) \mid y_{\xi}\left(t^{\prime}, \xi\right)>0 \text { for all } \tau_{n-1}(\xi)<t^{\prime}<t\right\},
$$

if there exists some $t>\tau_{n-1}(\xi)$ such that $y_{\xi}\left(t^{\prime}, \xi\right)>0$ for all $t^{\prime} \in\left(\tau_{n-1}(\xi), t\right)$ and $y_{\xi}(t, \xi)=0$. We set $\tau_{n}(\xi)=\infty$ otherwise. For convenience, we let $\tau_{0}(\xi)=0$ for all $\xi \in \mathbb{R}$. Then, as above, we impose

$$
\bar{h}\left(\tau_{n}(\xi), \xi\right)=(1-\alpha) \bar{h}\left(\tau_{n}(\xi)-0, \xi\right) \quad \text { and } \quad h\left(\tau_{n}(\xi), \xi\right)=h\left(\tau_{n}(\xi)-0, \xi\right) \text {. }
$$

We denote by $l_{j}$ the change in $\bar{h}$ due to the collision; that is,

$$
l_{j}(\xi)=\bar{h}\left(\tau_{j}(\xi)-0, \xi\right)-\bar{h}\left(\tau_{j}(\xi), \xi\right)=\alpha \bar{h}\left(\tau_{j}(\xi)-0, \xi\right) .
$$

REMARK 1. The sequence $\tau_{n}(\xi)$ is increasing and can a priori accumulate. However, we will show that this does not happen; see Corollary 19.

DEFINITION 1. An $\alpha$-dissipative solution in Lagrangian coordinates is given by the functions $\left(y, U, y_{\xi}, U_{\xi}, \bar{h}, h, r\right)$ such that

$$
\begin{gathered}
y-\operatorname{Id} \in L^{\infty}\left([0, T], W^{1, \infty}(\mathbb{R})\right), \quad U \in L^{\infty}\left([0, T], H^{1}(\mathbb{R})\right), \\
y_{\xi}-1, U_{\xi}, r \in W^{1, \infty}\left([0, T], L^{2}(\mathbb{R}) \cap L^{\infty}(\mathbb{R})\right) \\
\bar{h} \in L^{\infty}\left([0, T], L^{2}(\mathbb{R})\right), \quad h \in W^{1, \infty}\left([0, T], L^{1}(\mathbb{R}) \cap L^{\infty}(\mathbb{R})\right),
\end{gathered}
$$

and measurable functions $\tau_{1}(\xi)<\tau_{2}(\xi)<\cdots$, either finitely many or $\tau_{n}(\xi) \rightarrow \infty$ as $n \rightarrow \infty$, given by (28) and (29), which satisfy, for almost every $\xi \in \mathbb{R}$,

$$
\begin{gathered}
y_{t}(t, \xi)=U(t, \xi), \\
U_{t}(t, \xi)=-Q(t, \xi), \\
y_{t, \xi}(t, \xi)=U_{\xi}(t, \xi), \\
U_{t, \xi}(t, \xi)=\frac{1}{2} \bar{h}(t, \xi)+\left(U^{2}(t, \xi)-P(t, \xi)\right) y_{\xi}(t, \xi), \\
h_{t}(t, \xi)=2\left(U^{2}(t, \xi)-P(t, \xi)\right) U_{\xi}(t, \xi), \\
\bar{h}_{t}(t, \xi)=h_{t}(t, \xi), \\
r_{t}(t, \xi)=0,
\end{gathered}
$$

for $t \in\left[\tau_{n-1}(\xi), \tau_{n}(\xi)\right)$ and

$$
\begin{gathered}
X\left(\tau_{n}(\xi), \xi\right)=X\left(\tau_{n}(\xi)-0, \xi\right), \\
\bar{h}\left(\tau_{n}(\xi), \xi\right)=(1-\alpha) \bar{h}\left(\tau_{n}(\xi)-0, \xi\right),
\end{gathered}
$$


for $X=\left(y, U, y_{\xi}, U_{\xi}, h, r\right)$. In (32), the functions $P$ and $Q$ are given by

$$
P(t, \xi)=\frac{1}{4} \int_{\mathbb{R}} e^{-|y(t, \xi)-y(t, \eta)|}\left(2 U^{2} y_{\xi}+\bar{h}\right)(t, \eta) d \eta
$$

and

$$
Q(t, \xi)=-\frac{1}{4} \int_{\mathbb{R}} \operatorname{sgn}(\xi-\eta) e^{-|y(t, \xi)-y(t, \eta)|}\left(2 U^{2} y_{\xi}+\bar{h}\right)(t, \eta) d \eta,
$$

respectively.

REMARK 2. Note that, due to the above considerations, we can represent $\bar{h}(t, \xi)$ in the following way:

$$
\bar{h}(t, \xi)=h(t, \xi)-\sum_{j=0}^{n} l_{j}(\xi), \quad \text { for } t \in\left[\tau_{n}(\xi), \tau_{n+1}(\xi)\right),
$$

where we recursively define $l_{j}(\xi)=\alpha\left(h\left(\tau_{j}(\xi), \xi\right)-\sum_{k=0}^{j-1} l_{k}(\xi)\right)$ for $j \in \mathbb{N}$, and $l_{0}(\xi)=h(0, \xi)-\bar{h}(0, \xi) \geqslant 0$ and $\tau_{0}(\xi)=0$. In particular, we have $0 \leqslant \bar{h}(t, \xi) \leqslant h(t, \xi)$.

REMARK 3. We will here try to explain the strategy behind the lengthy existence proof in Lagrangian variables. Our starting point is the formulation (27) in Lagrangian variables. We replace the mixed derivatives $y_{t, \xi}$ and $U_{t, \xi}$ by new variables, namely $q=y_{\xi}$ and $w=U_{\xi}$, which turns (27) into a system of ordinary differential equations. We show the existence of a solution by an iterative argument, as part of the proof of Theorem 15. To secure a global solution, and to make sure that the underlying structure is preserved (for example, that the functions $q$ and $w$ satisfy $q=y_{\xi}$ and $w=U_{\xi}$, respectively), we have to restrict the set of initial data to the set $\mathcal{G}$; see Definition 3. The existence of global solutions then follows in the standard way by showing that the solution remains bounded. This would then yield the solution in Lagrangian variables in the conservative case. However, to construct the $\alpha$-dissipative solutions we need to monitor $y_{\xi}(t$, $\xi)$ carefully as a function of $t$ for each fixed $\xi$. At the first occasion when $y_{\xi}(t$, $\xi)=0$, that is, when $t=\tau_{1}(\xi)$, we read off the values of the dependent variables, and scale the variable $\bar{h}$ (which equals $h$ up to $\tau_{1}(\xi)$ ) by the factor $1-\alpha$. The system of ordinary differential equations is then restarted at $t=\tau_{1}(\xi)$ and runs according to (32) until the next time $y_{\xi}(t, \xi)$ vanishes. Again the function $\bar{h}$ is rescaled, and the system restarted. This construction is performed for each $\xi \in \mathbb{R}$. As the system of ordinary differential equations is discontinuous, the global existence proof requires careful estimates; see Lemmas 5, 6, 8, 10-13, 16. 
The function $g$, introduced below in Definition 2, plays a subtle role in our considerations. It is used in Lemma 10 , when identifying $\kappa_{1-\gamma}$ (see (56)), as the set of points which will experience wave breaking in the near future. However, it will play an even more vital role in the (future) construction of a Lipschitz metric for this system; see, for example, $[6,16]$. A close look at $g$ and $\bar{h}$ reveals that the function $\bar{h}$ drops suddenly at breaking time, while the function $g$ models the loss of energy in a continuous way. Thus $g$ will play a major role in (future) investigations about the stability of solutions.

We introduce the following notation for the Banach spaces that are frequently used. Let

$$
E=L^{2}(\mathbb{R}) \cap L^{\infty}(\mathbb{R}),
$$

together with the norm

$$
\|f\|_{E}=\|f\|_{L^{2}}+\|f\|_{L^{\infty}}
$$

and let

$$
\begin{gathered}
W=\left[L^{2}(\mathbb{R})\right]^{4}, \quad \bar{W}=E^{4}, \\
V=L^{\infty}(\mathbb{R}) \times L^{2}(\mathbb{R}) \times W, \quad \bar{V}=L^{\infty}(\mathbb{R}) \times E \times \bar{W} .
\end{gathered}
$$

For any function $f \in C([0, T], B)$ for $T \geqslant 0$ and $B$ a normed space, we denote

$$
\|f\|_{L_{T}^{1} B}=\int_{0}^{T}\|f(t, \cdot)\|_{B} d t \quad \text { and } \quad\|f\|_{L_{T}^{\infty} B}=\sup _{t \in[0, T]}\|f(t, \cdot)\|_{B} .
$$

Definition 2. For $x=\left(x_{1}, \ldots, x_{7}\right) \in \mathbb{R}^{7}$, we define the functions $g_{1}, g_{2}$, $g: \mathbb{R}^{7} \rightarrow \mathbb{R}$ by

$$
\begin{aligned}
& g_{1}(x)=\left|x_{4}\right|+2 x_{3}, \\
& g_{2}(x)=x_{3}+x_{5},
\end{aligned}
$$

and

$$
g(x)= \begin{cases}\alpha g_{1}(x)+(1-\alpha) g_{2}(x) & \text { if } x \in \Omega_{1}, \\ g_{2}(x) & \text { otherwise, }\end{cases}
$$

where $\Omega_{1}$ is the set where $g_{1} \leqslant g_{2}, x_{4}$ is nonpositive, and $x_{7}=0$; thus

$$
\Omega_{1}=\left\{x \in \mathbb{R}^{7}|| x_{4} \mid+2 x_{3} \leqslant x_{3}+x_{5}, x_{4} \leqslant 0 \text {, and } x_{7}=0\right\} .
$$

We identify $x=\left(x_{1}, \ldots, x_{7}\right)$ with $\Theta=\left(y, U, y_{\xi}, U_{\xi}, \bar{h}, h, r\right)$. 
REMARK 4. In the case of conservative solutions (that is, $\alpha=0$ ), we have $0<g(\Theta)(t, \xi)=g_{2}(\Theta)(t, \xi)$ and $h(t, \xi)=\bar{h}(t, \xi)$ for all $\xi \in \mathbb{R}$ and $t \in \mathbb{R}$. In the case of dissipative solutions (that is, $\alpha=1$ ), we infer $0<g(\Theta)(t, \xi)$ and $h(t, \xi)=\bar{h}(t, \xi)$ before wave breaking, while $0=g(\Theta)(t, \xi)$ and $\bar{h}(t, \xi)=0$ thereafter. The function $g(\Theta)(t, \xi)$ is introduced in such a way that it describes the loss of energy in a continuous way, in contrast to $\bar{h}(t, \xi)$, which drops suddenly at wave breaking.

Definition 3. The set $\mathcal{G}$ consists of all $\Theta=\left(y, U, y_{\xi}, U_{\xi}, \bar{h}, h, r\right)$ such that

$$
\begin{gathered}
X=\left(\zeta, U, \zeta_{\xi}, U_{\xi}, h, r\right) \in \bar{V}, \\
g(\Theta)-1 \in E, \\
h \in L^{1}(\mathbb{R}), \\
y_{\xi} \geqslant 0, h \geqslant 0, \bar{h} \geqslant 0 \text { almost everywhere, } \\
\lim _{\xi \rightarrow-\infty} \zeta(\xi)=0, \\
\frac{1}{y_{\xi}+h} \in L^{\infty}(\mathbb{R}), \\
y_{\xi} \bar{h}=U_{\xi}^{2}+r^{2} \text { almost everywhere, } \\
h \geqslant \bar{h} \text { almost everywhere, }
\end{gathered}
$$

where we denote $y(\xi)=\zeta(\xi)+\xi$.

The condition (37e) will be valid as long as the solution exists, since in that case we must have $\lim _{\xi \rightarrow-\infty} U(t, \xi)=0$ by construction. In addition, it should be noted that, due to the definition of $g(\Theta)$, the relation $(37 b)$ is valid for any $\Theta$ that satisfies $(37 a)$, since $0 \leqslant \bar{h} \leqslant h$.

Making the identifications $q=y_{\xi}$ and $w=U_{\xi}$, we obtain

$$
\begin{gathered}
y_{t}=U, \\
U_{t}=-Q(\Theta), \\
q_{t}=w, \\
w_{t}=\frac{1}{2} \bar{h}+\left(U^{2}-P(\Theta)\right) q, \\
h_{t}=2\left(U^{2}-P(\Theta)\right) w, \\
r_{t}=0,
\end{gathered}
$$

where $P(\Theta)$ and $Q(\Theta)$ are given by

$$
P(t, \xi)=\frac{1}{4} \int_{\mathbb{R}} e^{-|y(t, \xi)-y(t, \eta)|}\left(2 U^{2} q+\bar{h}\right)(t, \eta) d \eta,
$$


and

$$
Q(t, \xi)=-\frac{1}{4} \int_{\mathbb{R}} \operatorname{sgn}(\xi-\eta) e^{-|y(t, \xi)-y(t, \eta)|}\left(2 U^{2} q+\bar{h}\right)(t, \eta) d \eta,
$$

respectively.

The definition of $\tau_{1}$ given by (28) (after replacing $y_{\xi}$ by the corresponding variable $q)$ is not appropriate for $q \in C\left([0, T], L^{\infty}(\mathbb{R})\right)$, and, in addition, it is not clear from this definition if $\tau_{1}$ is measurable. Thus we replace this definition by the following one. Let $\left\{t_{i}\right\}_{i=1}^{\infty}$ be a dense countable subset of $[0, T]$. Define

$$
A_{t}=\bigcup_{n \in \mathbb{N}} \bigcap_{t_{i} \leqslant t}\left\{\xi \in \mathbb{R} \mid q\left(t_{i}, \xi\right)>\frac{1}{n}\right\} \text {. }
$$

The sets $A_{t}$ are measurable for all $t$, and we have $A_{t^{\prime}} \subset A_{t}$ for $t \leqslant t^{\prime}$. We consider a dyadic partition of the interval $[0, T]$ (that is, for each $n$, we consider the set $\left.\left\{2^{-n} i T\right\}_{i=0}^{2^{n}}\right)$, and set

$$
\tau_{1}^{n}(\xi)=\sum_{i=0}^{2^{n}} \frac{i T}{2^{n}} \chi_{i, n}(\xi)
$$

where $\chi_{i, n}$ is the indicator function of the set $A_{2^{-n}{ }_{i T}} \backslash A_{2^{-n}(i+1) T}$. The function $\tau_{1}^{n}$ is by construction measurable. One can check that $\tau_{1}^{n}(\xi)$ is increasing with respect to $n$; it is also bounded by $T$. Hence, we can define

$$
\tau_{1}(\xi)=\lim _{n \rightarrow \infty} \tau_{1}^{n}(\xi),
$$

and $\tau_{1}$ is a measurable function. The next lemma gives the main property of $\tau_{1}$.

LEMMA 5. If, for every $\xi \in \mathbb{R}, q(t, \xi)$ is positive and continuous with respect to time, then

$$
\tau_{1}(\xi)= \begin{cases}\sup \left\{t \in \mathbb{R}^{+} \mid q\left(t^{\prime}, \xi\right)>0 \text { for all } 0<t^{\prime}<t\right\} & \text { if }\{\cdots\} \neq \emptyset \\ \infty & \text { otherwise }\end{cases}
$$

that is, we retrieve Definition (28).

Proof. See [29].

One can represent $\tau_{n}(\xi)$ with $n=2,3, \ldots$, similarly. Indeed, let $\left\{t_{i}\right\}_{i=1}^{\infty}$ be a dense countable subset of $[0, T]$. Define inductively

$$
A_{n, t}=\bigcup_{m \in \mathbb{N}} \bigcap_{t_{i} \leqslant t}\left\{\xi \in \mathbb{R} \mid \tau_{n-1}(\xi) \leqslant t_{i}, q\left(t_{i}, \xi\right)>\frac{1}{m}\right\}, \quad n=2,3, \ldots
$$


As before, the sets $A_{n, t}$ are measurable for all $t$, and, in particular, $A_{n, t^{\prime}} \subset A_{n, t}$ for $t \leqslant t^{\prime}$. We consider a dyadic partition of the interval [0,T], and set

$$
\tau_{n}^{m}(\xi)=\sum_{i=0}^{2^{m}} \frac{i T}{2^{m}} \chi_{i, n, m}(\xi)
$$

where $\chi_{i, n, m}$ is the indicator function of the set $A_{n, 2^{-m} i T} \backslash A_{n, 2^{-m}(i+1) T}$. The function $\tau_{n}^{m}(\xi)$ is by construction measurable. One can check that $\tau_{n}^{m}(\xi)$ is increasing with respect to $m$, and bounded by $T$. Hence, we define

$$
\tau_{n}(\xi)=\lim _{m \rightarrow \infty} \tau_{n}^{m}(\xi),
$$

and $\tau_{n}(\xi)$ is a measurable function. Concluding as in the proof of Lemma 5, one obtains the following result.

LEMMA 6. If, for every $\xi \in \mathbb{R}, q(t, \xi)$ is positive and continuous with respect to time, then

$$
\tau_{n}(\xi)= \begin{cases}\sup \left\{t \in\left(\tau_{n-1}(\xi), \infty\right) \mid q\left(t^{\prime}, \xi\right)>0\right. & \\ \text { for all } \left.t^{\prime} \in\left(\tau_{n-1}(\xi), t\right)\right\} & \text { if }\{\cdots\} \neq \emptyset \\ \infty & \text { otherwise }\end{cases}
$$

for $n=2,3, \ldots$

REMARK 7. In the case of conservative solutions, we actually do not need to define $\tau_{j}(\xi)$ for $\xi \in \mathbb{R}$, because we do not redefine our system (32) after wave breaking.

So far, we have identified $q$ with $y_{\xi}$. However, $y_{\xi}$ does not decay fast enough at infinity to belong to $L^{2}(\mathbb{R})$, but $y_{\xi}-1=\zeta_{\xi}$ will be in $L^{2}(\mathbb{R})$, and we therefore introduce $v=q-1$. In the case of conservative solutions, we know that $Q(\Theta)$ and $P(\Theta)$ are Lipschitz continuous on bounded sets and that $Q(\Theta)$ and $P(\Theta)$ can be bounded by a constant depending on the bounded set. A slightly different result is true when describing $\alpha$-dissipative solutions. Define

$$
\begin{array}{r}
B_{M}=\left\{\Theta \mid\|X\|_{\bar{V}}+\|h\|_{L^{1}}+\left\|\frac{1}{q+h}\right\|_{L^{\infty}} \leqslant M,\right. \\
\left.q \bar{h}=w^{2}+r^{2}, \bar{h} \leqslant h, \text { and } q, \bar{h} \geqslant 0 \text { a.e. }\right\} .
\end{array}
$$

In addition, it should be pointed out that for any $\Theta \in C\left([0, T], B_{M}\right)$ the set of all points which experience wave breaking within a finite time interval $[0, T]$ is 
bounded, since

$$
\begin{aligned}
\operatorname{meas}(\{\xi \in \mathbb{R} \mid q(t, \xi)=0\}) & \leqslant \int_{\mathbb{R}} \frac{h}{q+h}(t, \xi) d \xi \\
& \leqslant\left\|\frac{1}{q+h}\right\|_{L_{T}^{\infty} L^{\infty}}\|h\|_{L_{T}^{\infty} L^{1}} \leqslant C(M),
\end{aligned}
$$

for all $t \in[0, T]$, where $C(M)$ denotes some constant depending only on $M$.

Lemma 8. (i) For all $\Theta \in C\left([0, T], B_{M}\right)$, we have

$$
\|Q(\Theta)\|_{L_{T}^{\infty} E}+\|P(\Theta)\|_{L_{T}^{\infty} E} \leqslant C(M)
$$

for a constant $C(M)$ which depends only on $M$.

(ii) For any $\Theta$ and $\tilde{\Theta}$ in $C\left([0, T], B_{M}\right)$, we have

$$
\begin{aligned}
& \|Q(\Theta)-Q(\tilde{\Theta})\|_{L_{T}^{1} E}+\|P(\Theta)-P(\tilde{\Theta})\|_{L_{T}^{1} E} \\
& \quad \leqslant C(M)\left(T\|X-\tilde{X}\|_{L_{T}^{\infty} \bar{V}}+\int_{0}^{T} \int_{\mathbb{R}}|\bar{h}(t, \xi)-\overline{\tilde{h}}(t, \xi)| d \xi d t\right) .
\end{aligned}
$$

Here, $C(M)$ denotes a constant which depends only on $M$.

Proof. We will establish only the estimates for $P(\Theta)$, as those for $Q(\Theta)$ can be obtained using the same methods with only slight modifications. The main tool for proving the stated estimates will be Young's inequality, which we recall here for the sake of completeness. For any $f \in L^{p}(\mathbb{R})$ and $g \in L^{q}(\mathbb{R})$ with $1 \leqslant p, q$, $r \leqslant \infty$, we have

$$
\|f \star g\|_{L^{r}} \leqslant\|f\|_{L^{p}}\|g\|_{L^{q}}, \quad \text { if } 1+\frac{1}{r}=\frac{1}{p}+\frac{1}{q} .
$$

(i) By definition, we have

$$
P(\Theta)(t, \xi)=\frac{1}{4} \int_{\mathbb{R}} e^{-|y(t, \xi)-y(t, \eta)|}\left(2 U^{2} q+\bar{h}\right)(t, \eta) d \eta .
$$

So far, we do not know if $y(t, \xi)$ is an increasing function or not; thus we will split the integral above into three, as follows. By assumption, we have that $\|y(t, \xi)-\xi\|_{L_{T}^{\infty} L^{\infty}} \leqslant M$; thus

$$
\begin{aligned}
(\xi-\eta)-2 M & \leqslant y(t, \xi)-y(t, \eta)=(y(t, \xi)-\xi)+(\xi-\eta)-(y(t, \eta)-\eta) \\
& \leqslant(\xi-\eta)+2 M,
\end{aligned}
$$


and, in particular,

$$
\begin{array}{ll}
y(t, \xi)-y(t, \eta) \geqslant 0 & \text { if } \eta \leqslant \xi-2 M, \\
y(t, \xi)-y(t, \eta) \leqslant 0 & \text { if } \eta \geqslant \xi+2 M .
\end{array}
$$

Hence, we can rewrite (48) as

$$
\begin{aligned}
P(\Theta)(t, \xi)= & \frac{1}{4} \int_{-\infty}^{\xi-2 M} e^{-(y(t, \xi)-y(t, \eta))}\left(2 U^{2} q+\bar{h}\right)(t, \eta) d \eta \\
& +\frac{1}{4} \int_{\xi-2 M}^{\xi+2 M} e^{-|y(t, \xi)-y(t, \eta)|}\left(2 U^{2} q+\bar{h}\right)(t, \eta) d \eta \\
& +\frac{1}{4} \int_{\xi+2 M}^{\infty} e^{-(y(t, \eta)-y(t, \xi))}\left(2 U^{2} q+\bar{h}\right)(t, \eta) d \eta \\
= & I_{1}(t, \xi)+I_{2}(t, \xi)+I_{3}(t, \xi) .
\end{aligned}
$$

Let $f(\xi)=\chi_{\{\xi>2 M\}} e^{-\xi}$. Then we have

$$
\begin{aligned}
\left\|I_{1}(t, \xi)\right\|_{L_{T}^{\infty} E} & =\left\|\frac{1}{4} \int_{-\infty}^{\xi-2 M} e^{-\zeta(t, \xi)} e^{-(\xi-\eta)} e^{\zeta(t, \eta)}\left(2 U^{2} q+\bar{h}\right)(t, \eta) d \eta\right\|_{L_{T}^{\infty} E} \\
& \leqslant \frac{1}{4} e^{\|\zeta\|_{L_{T}^{\infty} L^{\infty}}}\left\|\left(f \star\left[e^{\zeta}\left(2 U^{2} q+\bar{h}\right)\right]\right)(t, \xi)\right\|_{L_{T}^{\infty} E} \\
& \leqslant C(M)\left(\|f\|_{L^{1}}+\|f\|_{L^{2}}\right)\left\|e^{\zeta}\left(2 U^{2} q+\bar{h}\right)\right\|_{L_{T}^{\infty} L^{2}} \\
& \leqslant C(M),
\end{aligned}
$$

since $\bar{h}(t, \xi) \leqslant h(t, \xi)$. Similarly, one can estimate $\left\|I_{3}(t, \xi)\right\|_{L_{T}^{\infty} E}$ by replacing the function $f(\xi)$ by the function $g(\xi)=\chi_{\{\xi<-2 M\}} e^{\xi}$. As far as $I_{2}(t, \xi)$ is concerned, we conclude as follows:

$$
\begin{aligned}
\left\|I_{2}(t, \xi)\right\|_{L_{T}^{\infty} E} & \\
\leqslant & \left\|\frac{1}{4} \int_{\xi-2 M}^{\xi+2 M} e^{-|y(t, \xi)-y(t, \eta)|}\left(2 U^{2} q+\bar{h}\right)(t, \eta) d \eta\right\|_{L_{T}^{\infty} E} \\
\leqslant & \left\|\frac{1}{4} \int_{\xi-2 M}^{\xi+2 M}\left(e^{-(y(t, \xi)-y(t, \eta))}+e^{-(y(t, \eta)-y(t, \xi))}\right)\left(2 U^{2} q+\bar{h}\right)(t, \eta) d \eta\right\|_{L_{T}^{\infty} E} \\
\leqslant & \left\|\frac{1}{4} \int_{\xi-2 M}^{\xi+2 M} e^{-(y(t, \xi)-y(t, \eta))}\left(2 U^{2} q+\bar{h}\right)(t, \eta) d \eta\right\|_{L_{T}^{\infty} E} \\
& +\left\|\frac{1}{4} \int_{\xi-2 M}^{\xi+2 M} e^{-(y(t, \eta)-y(t, \xi))}\left(2 U^{2} q+\bar{h}\right)(t, \eta) d \eta\right\|_{L_{T}^{\infty} E}
\end{aligned}
$$


Following closely the argument we used for $I_{1}(t, \xi)$ yields

$$
\left\|I_{2}(t, \xi)\right\|_{L_{T}^{\infty} E} \leqslant C(M) .
$$

(ii) As before, we split the integral into three parts, and investigate each of them separately. We start with

$$
\begin{aligned}
B_{1}(t, \xi)= & \frac{1}{4} \int_{-\infty}^{\xi-2 M}\left(e^{-(y(t, \xi)-y(t, \eta))}\left(2 U^{2} q+\bar{h}\right)(t, \eta)\right. \\
& \left.-e^{-(\tilde{y}(t, \xi)-\tilde{y}(t, \eta))}\left(2 \tilde{U}^{2} \tilde{q}+\overline{\tilde{h}}\right)(t, \eta)\right) d \eta \\
= & \frac{1}{4}\left(e^{-\zeta(t, \xi)}-e^{-\tilde{\zeta}(t, \xi)}\right) \int_{-\infty}^{\xi-2 M} e^{-(\xi-\eta)} e^{\zeta(t, \eta)}\left(2 U^{2} q+\bar{h}\right)(t, \eta) d \eta \\
& +\frac{1}{4} e^{-\tilde{\zeta}(t, \xi)} \int_{-\infty}^{\xi-2 M} e^{-(\xi-\eta)}\left(e^{\zeta(t, \eta)} 2 U^{2} q(t, \eta)-e^{\tilde{\zeta}(t, \eta)} 2 \tilde{U}^{2} \tilde{q}(t, \eta)\right) d \eta \\
& +\frac{1}{4} e^{-\tilde{\zeta}(t, \xi)} \int_{-\infty}^{\xi-2 M} e^{-(\xi-\eta)}\left(e^{\zeta(t, \eta)} \bar{h}(t, \eta)-e^{\tilde{\zeta}(t, \eta)} \overline{\tilde{h}}(t, \eta)\right) d \eta .
\end{aligned}
$$

Let $f(\xi)=\chi_{\{\xi>2 M\}} e^{-\xi}$; then

$$
\begin{aligned}
\left\|B_{1}(t, \xi)\right\|_{L_{T}^{1} E} \leqslant & C(M) T\|\zeta-\tilde{\zeta}\|_{L_{T}^{\infty} E}+C(M) T\left(\|f\|_{L^{1}}+\|f\|_{L^{2}}\right)\|X-\tilde{X}\|_{L_{T}^{\infty} V} \\
& +C(M)\left(\|f\|_{L^{\infty}}+\|f\|_{L^{2}}\right) \\
& \times\left(T\|X-\tilde{X}\|_{L_{T}^{\infty} V}+\int_{0}^{T} \int_{\mathbb{R}}|\bar{h}(t, \xi)-\overline{\tilde{h}}(t, \xi)| d \xi d t\right) \\
\leqslant & C(M)\left(T\|X-\tilde{X}\|_{L_{T}^{\infty} V}+\int_{0}^{T} \int_{\mathbb{R}}|\bar{h}(t, \xi)-\overline{\tilde{h}}(t, \xi)| d \xi d t\right) .
\end{aligned}
$$

$B_{3}(t, \xi)$, which corresponds to $I_{3}(t, \xi)$ in (i), can be investigated similarly. As far as $B_{2}(t, \xi)$ is concerned, we have

$$
\begin{aligned}
B_{2}(t, \xi)= & \frac{1}{4} \int_{\xi-2 M}^{\xi+2 M}\left(e^{-|y(t, \xi)-y(t, \eta)|}\left(2 U^{2} q+\bar{h}\right)(t, \eta)\right. \\
& \left.-e^{-|\tilde{y}(t, \xi)-\tilde{y}(t, \eta)|}\left(2 \tilde{U}^{2} \tilde{q}+\overline{\tilde{h}}\right)(t, \eta)\right) d \eta \\
= & \frac{1}{4} \int_{\xi-2 M}^{\xi+2 M}\left(e^{-|y(t, \xi)-y(t, \eta)|}-e^{-|\tilde{y}(t, \xi)-\tilde{y}(t, \eta)|}\right)\left(2 U^{2} q+\bar{h}\right)(t, \eta) d \eta \\
& +\frac{1}{4} \int_{\xi-2 M}^{\xi+2 M} e^{-|\tilde{y}(t, \xi)-\tilde{y}(t, \eta)|}\left(2 U^{2} q+\bar{h}-2 \tilde{U}^{2} \tilde{q}-\overline{\tilde{h}}\right)(t, \eta) d \eta \\
= & \frac{1}{4} \int_{\xi-2 M}^{\xi+2 M} e^{-|y(t, \xi)-y(t, \eta)|}
\end{aligned}
$$




$$
\begin{aligned}
& \times\left(1-e^{-|\tilde{y}(t, \xi)-\tilde{y}(t, \eta)|+|y(t, \xi)-y(t, \eta)|}\right)\left(2 U^{2} q+\bar{h}\right)(t, \eta) d \eta \\
& +\int_{\xi-2 M}^{\xi+2 M} e^{-|\tilde{y}(t, \xi)-\tilde{y}(t, \eta)|} 2\left(U^{2} q-\tilde{U}^{2} \tilde{q}\right)(t, \eta) d \eta \\
& +\int_{\xi-2 M}^{\xi+2 M} e^{-|\tilde{y}(t, \xi)-\tilde{y}(t, \eta)|}(\bar{h}-\overline{\tilde{h}})(t, \eta) d \eta \\
& =B_{21}(t, \xi)+B_{22}(t, \xi)+B_{23}(t, \xi) .
\end{aligned}
$$

$\left\|B_{22}(t, \xi)\right\|_{L_{T}^{1} E}$ and $\left\|B_{23}(t, \xi)\right\|_{L_{T}^{1} E}$ can be estimated using Young's inequality, while $\left\|B_{21}(t, \xi)\right\|_{L_{T}^{1} E}$ requires more careful estimates. Since $\xi-2 M \leqslant \eta \leqslant$ $\xi+2 M$, we have

$$
\begin{aligned}
& \|y(t, \xi)-y(t, \eta)|-| \tilde{y}(t, \xi)-\tilde{y}(t, \eta)\| \\
& \quad \leqslant|y(t, \xi)-\tilde{y}(t, \xi)|+|y(t, \eta)-\tilde{y}(t, \eta)| \leqslant 2\|y-\tilde{y}\|_{L_{T}^{\infty} L^{\infty}}
\end{aligned}
$$

and

$$
\begin{aligned}
& \|y(t, \xi)-y(t, \eta)|-| \tilde{y}(t, \xi)-\tilde{y}(t, \eta)\| \\
& \quad \leqslant|y(t, \xi)-y(t, \eta)|+|\tilde{y}(t, \xi)-\tilde{y}(t, \eta)| \\
& \quad \leqslant 4\|y-\operatorname{Id}\|_{L_{T}^{\infty} L^{\infty}}+2|\xi-\eta| \leqslant 8 M .
\end{aligned}
$$

Hence

$$
\begin{aligned}
\left|1-e^{-|\tilde{y}(t, \xi)-\tilde{y}(t, \eta)|+|y(t, \xi)-y(t, \eta)|}\right| & \leqslant\left|\int_{-|\tilde{y}(t, \xi)-\tilde{y}(t, \eta)|+|y(t, \xi)-y(t, \eta)|}^{0} e^{x} d x\right| \\
& \leqslant C(M)\|y-\tilde{y}\|_{L_{T}^{\infty} L^{\infty}}
\end{aligned}
$$

and

$$
\begin{aligned}
& \left\|B_{21}(t, \xi)\right\|_{L_{T}^{1} E} \\
& \leqslant C(M)\|y-\tilde{y}\|_{L_{T}^{\infty} L^{\infty}}\left\|\frac{1}{4} \int_{\xi-2 M}^{\xi+2 M} e^{-|y(t, \xi)-y(t, \eta)|}\left(2 U^{2} q+\bar{h}\right)(t, \eta) d \eta\right\|_{L_{T}^{1} E} \\
& \leqslant C(M) T\|y-\tilde{y}\|_{L_{T}^{\infty} L^{\infty}}\left(\left\|\frac{1}{4} \int_{\xi-2 M}^{\xi+2 M} e^{-(y(t, \xi)-y(t, \eta))}\left(2 U^{2} q+\bar{h}\right)(t, \eta) d \eta\right\|_{L_{T}^{\infty} E}\right. \\
& \left.\quad+\left\|\frac{1}{4} \int_{\xi-2 M}^{\xi+2 M} e^{-(y(t, \eta)-y(t, \xi))}\left(2 U^{2} q+\bar{h}\right)(t, \eta) d \eta\right\|_{L_{T}^{\infty} E}\right) \\
& \leqslant C(M) T\|y-\tilde{y}\|_{L_{T}^{\infty} L^{\infty} .}
\end{aligned}
$$

Thus, putting everything together, we have

$$
\left\|B_{2}(t, \xi)\right\|_{L_{T}^{1} E} \leqslant C(M)\left(T\|X-\tilde{X}\|_{L_{T}^{\infty} V}+\int_{0}^{T} \int_{\mathbb{R}}|\bar{h}(t, \xi)-\overline{\tilde{h}}(t, \xi)| d \xi d t\right) .
$$


REMARK 9. (i) In the case of conservative solutions, that is, $\alpha=0$, we have $h(t, \xi)=\bar{h}(t, \xi)$, and hence

$$
\int_{0}^{T} \int_{\mathbb{R}}|\bar{h}(t, \xi)-\overline{\tilde{h}}(t, \xi)| d \xi d t \leqslant T C(M)\|X-\tilde{X}\|_{L_{T}^{\infty} \bar{V}},
$$

after using that $h=U_{\xi}^{2}+r^{2}-h \zeta_{\xi}$ together with the Cauchy-Schwarz inequality. (ii) In the case of dissipative solutions, that is, $\alpha=1$, we get, since $\bar{h}(t, \xi)=0$ for $t \geqslant \tau_{1}(\xi)$, that

$$
\begin{aligned}
& \int_{0}^{T} \int_{\mathbb{R}}|\bar{h}(t, \xi)-\overline{\tilde{h}}(t, \xi)| d \xi d t \leqslant C(M)\left(T\|X-\tilde{X}\|_{L_{T}^{\infty} \bar{V}}\right. \\
& \left.\quad+\int_{\mathbb{R}}\left(\int_{\tau_{1}}^{\tilde{\tau}_{1}} \tilde{h}(t, \xi) \chi_{\left\{\tilde{\tau}_{1}>\tau_{1}\right\}}(\xi) d t+\int_{\tilde{\tau}_{1}}^{\tau_{1}} h(t, \xi) \chi_{\left\{\tau_{1}>\tilde{\tau}_{1}\right\}}(\xi) d t\right) d \xi\right) .
\end{aligned}
$$

Here, we used the same argument as in (i) together with an application of Fubini's theorem. In particular, this means that the norm estimates here imply the ones in [18], where the dissipative case is studied, and vice versa.

To show short-time existence of solutions, we will use an iteration argument for the following system of ordinary differential equations. Denote generically $(\zeta, U, q, w, \bar{h}, h, r)$ by $\Theta,(\zeta, U, q, w, h, r)$ by $X$, and $(q, w, h, r)$ by $Z$; thus $X=(\zeta, U, Z)$. Then, we define the mapping

$$
\mathcal{P}: C\left([0, T], B_{M}\right) \rightarrow C\left([0, T], B_{M}\right)
$$

as follows: given $\Theta_{0} \in \mathcal{G} \cap B_{M_{0}}$ and $\Theta \in C\left([0, T], B_{M}\right)$, we can compute $P(\Theta)$ and $Q(\Theta)$ using (39) and (40). Then, we define $\tilde{\Theta}=\mathcal{P}(\Theta)$ as follows. Given $\xi \in \mathbb{R}$, we set $\tilde{\Theta}(0, \xi)=\Theta_{0}(\xi)$ and $\tilde{\Theta}(t, \xi)$ on $\left[\tilde{\tau}_{n}(\xi), \tilde{\tau}_{n+1}(\xi)\right]$ as the solution of the system of ordinary differential equations

$$
\begin{gathered}
\tilde{\zeta}_{t}(t, \xi)=\tilde{U}(t, \xi), \\
\tilde{U}_{t}(t, \xi)=-Q(\Theta)(t, \xi), \\
\tilde{q}_{t}(t, \xi)=\tilde{w}(t, \xi), \\
\tilde{w}_{t}(t, \xi)=\frac{1}{2} \tilde{\tilde{h}}(t, \xi)+\left(U^{2}(t, \xi)-P(\Theta)(t, \xi)\right) \tilde{q}(t, \xi), \\
\tilde{h}_{t}(t, \xi)=2\left(U^{2}(t, \xi)-P(\Theta)(t, \xi)\right) \tilde{w}(t, \xi), \\
\overline{\tilde{h}}_{t}(t, \xi)=\tilde{h}_{t}(t, \xi), \\
\tilde{r}_{t}(t, \xi)=0,
\end{gathered}
$$

which satisfies, at $t=\tilde{\tau}_{n}(\xi)$,

$$
\tilde{X}\left(\tilde{\tau}_{n}(\xi), \xi\right)=\tilde{X}\left(\tilde{\tau}_{n}(\xi)-0, \xi\right) \quad \text { and } \quad \overline{\tilde{h}}\left(\tilde{\tau}_{n}(\xi), \xi\right)=(1-\alpha) \overline{\tilde{h}}\left(\tilde{\tau}_{n}(\xi)-0, \xi\right) \text {. }
$$


We write $\overline{\tilde{Z}}_{t}=F(\Theta) \overline{\tilde{Z}}$, where $\overline{\tilde{Z}}=(\tilde{q}, \tilde{w}, \overline{\tilde{h}}, \tilde{r})$ for all times $t$, where no wave breaking occurs, that is, for $t \in\left[\tilde{\tau}_{n}(\xi), \tilde{\tau}_{n+1}(\xi)\right)$. So far, we have not excluded that the sequence $\tilde{\tau}_{n}(\xi)$ might have an accumulation point $\tilde{\tau}_{\infty}(\xi)$. Later on, we will see that this is not possible; see Lemma 12. If the sequence $\tilde{\tau}_{n}(\xi)$ were to have an accumulation point $\tilde{\tau}_{\infty}(\xi)$, we define $\tilde{\Theta}$ as the solution of

$$
\begin{gathered}
\tilde{y}_{t}(t, \xi)=\tilde{U}(t, \xi), \quad \tilde{U}_{t}(t, \xi)=-Q(t, \xi), \\
\tilde{q}_{t}(t, \xi)=\tilde{w}_{t}(t, \xi)=\overline{\tilde{h}}_{t}(t, \xi)=\tilde{r}_{t}(t, \xi)=0, \\
\tilde{h}(t, \xi)=\tilde{h}\left(\tilde{\tau}_{\infty}(\xi), \xi\right),
\end{gathered}
$$

for $t \in\left[\tilde{\tau}_{\infty}(\xi), T\right]$.

The following set will play a key role in the context of wave breaking, since it contains all points which will experience wave breaking in the near future,

$\kappa_{1-\gamma}=\left\{\xi \in \mathbb{R} \mid \frac{\bar{h}_{0}}{q_{0}+\bar{h}_{0}}(\xi) \geqslant 1-\gamma, w_{0}(\xi) \leqslant 0\right.$, and $\left.r_{0}(\xi)=0\right\}, \quad \gamma \in\left[0, \frac{1}{2}\right]$.

Note that

$$
\begin{aligned}
\frac{\bar{h}_{0}}{q_{0}+\bar{h}_{0}}(\xi) \geqslant 1-\gamma & \Longleftrightarrow \gamma \geqslant 1-\frac{\bar{h}_{0}}{q_{0}+\bar{h}_{0}}(\xi)=\frac{q_{0}}{q_{0}+\bar{h}_{0}}(\xi) \\
& \Longleftrightarrow(1-\gamma) q_{0}(\xi) \leqslant \gamma \bar{h}_{0}(\xi) \leqslant \gamma h_{0}(\xi),
\end{aligned}
$$

which implies that $\left(q_{0} /\left(q_{0}+h_{0}\right)\right)(\xi) \leqslant \gamma$, and hence $\left(h_{0} /\left(q_{0}+h_{0}\right)\right)(\xi) \geqslant 1-\gamma$. In particular, we have that

$$
\operatorname{meas}\left(\kappa_{1-\gamma}\right) \leqslant \frac{1}{1-\gamma} \int_{\mathbb{R}} \frac{h_{0}}{h_{0}+q_{0}}(\xi) d \xi \leqslant \frac{1}{1-\gamma}\left\|\frac{1}{q_{0}+h_{0}}\right\|_{L^{\infty}}\left\|h_{0}\right\|_{L^{1}},
$$

and therefore the set $\kappa_{1-\gamma}$ has finite measure if we choose $\gamma \in\left[0, \frac{1}{2}\right]$, and, in particular, meas $\left(\kappa_{1-\gamma}\right) \leqslant C(M)$.

LEMMA 10. Given $\Theta_{0} \in \mathcal{G} \cap B_{M_{0}}$ for some constant $M_{0}$, given $\Theta \in C\left([0, T], B_{M}\right)$, we denote by $\tilde{\Theta}=(\tilde{\zeta}, \tilde{U}, \tilde{v}, \tilde{w}, \tilde{\tilde{h}}, \tilde{h}, \tilde{r})=\mathcal{P}(\Theta)$ with initial data $\Theta_{0}$. Let

$$
\bar{M}=\|Q(\Theta)\|_{L_{T}^{\infty} L^{\infty}}+\|P(\Theta)\|_{L_{T}^{\infty} L^{\infty}}+\|U\|_{L_{T}^{\infty} L^{\infty}}^{2}
$$

Then the following statements hold.

(i) For all $t$, and almost all $\xi$,

$$
\tilde{q}(t, \xi) \geqslant 0, \quad \tilde{h}(t, \xi) \geqslant 0, \quad \overline{\tilde{h}}(t, \xi) \geqslant 0,
$$


and

$$
\tilde{q} \overline{\tilde{h}}=\tilde{w}^{2}+\tilde{r}^{2} \text {. }
$$

Thus, $\tilde{q}(t, \xi)=0$ implies that $\tilde{w}(t, \xi)=0$ and $\tilde{r}(t, \xi)=0$. Recall that $\tilde{q}=\tilde{v}+1$.

(ii) We have

$$
\left\|\frac{1}{\tilde{q}+\tilde{h}}(t, \cdot)\right\|_{L^{\infty}} \leqslant 2 e^{C(\bar{M}) T}\left\|\frac{1}{q_{0}+h_{0}}\right\|_{L^{\infty}},
$$

and

$$
\|(\tilde{q}+\tilde{h})(t, \cdot)\|_{L^{\infty}} \leqslant 2 e^{C(\bar{M}) T}\left\|q_{0}+h_{0}\right\|_{L^{\infty}},
$$

for all $t \in[0, T]$ and a constant $C(\bar{M})$ which depends only on $\bar{M}$. In particular, $\tilde{q}+\tilde{h}$ remains bounded strictly away from zero.

(iii) There exists a $\gamma \in\left(0, \frac{1}{2}\right)$ depending only on $\bar{M}$ such that, if $\xi \in \kappa_{1-\gamma}$, then $\tilde{\Theta}(t, \xi) \in \Omega_{1}$, where $\Omega_{1}$ is given in Definition 2 , for all $t \in\left[0, \min \left(\tilde{\tau}_{1}(\xi)\right.\right.$, $T)],(\tilde{q} /(\tilde{q}+\overline{\tilde{h}}))(t, \xi)$ is a decreasing function with respect to time for $t \in[0$, $\left.\min \left(\tilde{\tau}_{1}(\xi), T\right)\right]$ and $(\tilde{w} /(\tilde{q}+\overline{\tilde{h}}))(t, \xi)$ is an increasing function with respect to time for $t \in\left[0, \min \left(\tilde{\tau}_{1}(\xi), T\right)\right]$. Thus, we infer that

$$
\frac{w_{0}}{q_{0}+\bar{h}_{0}}(\xi) \leqslant \frac{\tilde{w}}{\tilde{q}+\overline{\tilde{h}}}(t, \xi) \leqslant 0 \quad \text { and } \quad 0 \leqslant \frac{\tilde{q}}{\tilde{q}+\overline{\tilde{h}}}(t, \xi) \leqslant \frac{q_{0}}{q_{0}+\bar{h}_{0}}(\xi)
$$

for $t \in\left[0, \min \left(\tilde{\tau}_{1}(\xi), T\right)\right]$. In addition, for $\gamma$ sufficiently small, depending only on $\bar{M}$ and $T$, we have

$$
\kappa_{1-\gamma} \subset\left\{\xi \in \mathbb{R} \mid 0 \leqslant \tilde{\tau}_{1}(\xi)<T\right\}
$$

(iv) Moreover, for any given $\gamma \in\left(0, \frac{1}{2}\right)$, there exists $\hat{T}>0$ such that

$$
\left\{\xi \in \mathbb{R} \mid 0<\tilde{\tau}_{1}(\xi)<\hat{T}\right\} \subset \kappa_{1-\gamma} .
$$

Proof. (i) Since $\Theta_{0} \in \mathcal{G}$, equations (58) and (59) hold for almost every $\xi \in \mathbb{R}$ at $t=0$. We consider such a $\xi$, and will drop it in the notation. From (54), we have, on the one hand,

$$
(\tilde{q} \overline{\tilde{h}})_{t}=\tilde{q}_{t} \overline{\tilde{h}}+\tilde{q} \overline{\tilde{h}}_{t}=\tilde{w} \tilde{\tilde{h}}+2\left(U^{2}-P(\Theta)\right) \tilde{w} \tilde{q}, \quad t \in\left(\tilde{\tau}_{n}, \tilde{\tau}_{n+1}\right),
$$

and, on the other hand,

$$
\left(\tilde{w}^{2}+\tilde{r}^{2}\right)_{t}=2 \tilde{w} \tilde{w}_{t}=\tilde{w} \overline{\tilde{h}}+2\left(U^{2}-P(\Theta)\right) \tilde{w} \tilde{q}, \quad t \in\left(\tilde{\tau}_{n}, \tilde{\tau}_{n+1}\right) .
$$

Thus,

$$
\left(\tilde{q} \overline{\tilde{h}}-\tilde{w}^{2}-\tilde{r}^{2}\right)_{t}=0,
$$


and, since $\tilde{q}(0) \overline{\tilde{h}}(0)=\tilde{w}^{2}(0)+\tilde{r}^{2}(0)$, we have $\tilde{q}(t) \overline{\tilde{h}}(t)=\tilde{w}^{2}(t)+\tilde{r}^{2}(t)$ for all $t \in\left[0, \tilde{\tau}_{1}\right)$. We show by induction that it holds for $t \in\left[\tilde{\tau}_{n-1}, \tilde{\tau}_{n}\right]$ for each $n \geqslant 1$, where $\tilde{\tau}_{0}=0$. We have $\tilde{q}\left(\tilde{\tau}_{n}-0\right)=q\left(\tilde{\tau}_{n}\right)=0$, so, by $(55)$,

$$
0=\tilde{q}\left(\tilde{\tau}_{n}\right) \overline{\tilde{h}}\left(\tilde{\tau}_{n}-0\right)=\tilde{w}^{2}\left(\tilde{\tau}_{n}\right)+\tilde{r}^{2}\left(\tilde{\tau}_{n}\right) .
$$

Hence, $\tilde{w}\left(\tilde{\tau}_{n}\right)=\tilde{r}\left(\tilde{\tau}_{n}\right)=0$, and

$$
\tilde{q}\left(\tilde{\tau}_{n}\right) \overline{\tilde{h}}\left(\tilde{\tau}_{n}\right)=0=\tilde{w}^{2}\left(\tilde{\tau}_{n}\right)+\tilde{r}^{2}\left(\tilde{\tau}_{n}\right),
$$

so (59) holds for $t=\tilde{\tau}_{n}$. By (65), we obtain that (59) holds also on the whole interval $\left[\tilde{\tau}_{n}, \tilde{\tau}_{n+1}\right]$. From the definition of $\tilde{\tau}_{1}$, we have that $\tilde{q}(t)>0$ on $\left[0, \tilde{\tau}_{1}\right)$, and $\tilde{q}\left(\tilde{\tau}_{1}\right)=\tilde{w}\left(\tilde{\tau}_{1}\right)=\tilde{r}\left(\tilde{\tau}_{1}\right)=0$ and $\overline{\tilde{h}}\left(\tau_{1}\right) \geqslant 0$. Hence $\tilde{w}(t)$ becomes positive at time $\tilde{\tau}_{1}$, and therefore $\tilde{q}(t)$ is increasing. Since, whenever $\tilde{q}(t)=0$, we have that $\tilde{w}$ changes sign from negative to positive, it follows that $\tilde{q}(t) \geqslant 0$ for $t \geqslant 0$. From (59) it follows that, for $t \in\left[0, \tilde{\tau}_{1}\right), \overline{\tilde{h}}(t)=\left(\left(\tilde{w}^{2}+\tilde{r}^{2}\right) / \tilde{q}\right)(t)$ and therefore $\overline{\tilde{h}}(t) \geqslant 0$. By the continuity of $\overline{\tilde{h}}$ (with respect to time), we have $\lim _{t \uparrow} \overline{\tilde{\tau}}(t) \geqslant 0$, and, using (55) and (59), we have $\overline{\tilde{h}}(t) \geqslant 0$ for all $t \in\left[0, \tilde{\tau}_{2}\right)$. The claim now follows by induction.

(ii) We consider a fixed $\xi$ that we suppress in the notation. We denote by $|\tilde{Z}|_{2}=$ $\left(\tilde{q}^{2}+\tilde{w}^{2}+\tilde{h}^{2}+\tilde{r}^{2}\right)^{1 / 2}$ the Euclidean norm of $\tilde{Z}=(\tilde{q}, \tilde{w}, \tilde{h}, \tilde{r})$. Since $0 \leqslant \tilde{\tilde{h}} \leqslant \tilde{h}$, we have

$$
\frac{d}{d t}|\tilde{Z}|_{2}^{-2}=-2|\tilde{Z}|_{2}^{-4} \tilde{Z} \frac{d \tilde{Z}}{d t} \leqslant C(\bar{M})|\tilde{Z}|_{2}^{-2}
$$

for a constant $C(\bar{M})$ which depends only on $\bar{M}$. Applying Gronwall's lemma, we obtain $|\tilde{Z}(t)|_{2}^{-2} \leqslant e^{C(\bar{M}) T}|Z(0)|_{2}^{-2}$. Hence,

$$
\frac{1}{\tilde{q}^{2}+\tilde{w}^{2}+\tilde{h}^{2}+\tilde{r}^{2}}(t) \leqslant e^{C(\bar{M}) T} \frac{1}{q_{0}^{2}+w_{0}^{2}+h_{0}^{2}+r_{0}^{2}} .
$$

Using (59), we have

$$
\tilde{q}^{2}+\tilde{w}^{2}+\tilde{h}^{2}+\tilde{r}^{2} \leqslant \tilde{q}^{2}+\tilde{q} \tilde{h}+\tilde{h}^{2}
$$

Hence, (66) yields

$$
\frac{1}{(\tilde{q}+\tilde{h})^{2}}(t) \leqslant \frac{1}{\tilde{q}^{2}+\tilde{q} \tilde{h}+\tilde{h}^{2}}(t) \leqslant e^{C(\bar{M}) T} \frac{1}{q_{0}^{2}+h_{0}^{2}} \leqslant 2 e^{C(\bar{M}) T} \frac{1}{\left(q_{0}+h_{0}\right)^{2}} .
$$

The second claim can be shown similarly. 
(iii) Let us consider a given $\xi \in \kappa_{1-\gamma}$. We are going to determine an upper bound on $\gamma$ depending only on $\bar{M}$ such that the conclusions of (iii) hold. For $\gamma$ small enough, we have $\Theta_{0}(\xi) \in \Omega_{1}$, as otherwise $g_{2}\left(\Theta_{0}(\xi)\right)=q_{0}(\xi)+\bar{h}_{0}(\xi)$, and

$$
1=\frac{g_{2}\left(\Theta_{0}(\xi)\right)}{q_{0}(\xi)+\bar{h}_{0}(\xi)}<\frac{-w_{0}(\xi)+2 q_{0}(\xi)}{q_{0}(\xi)+\bar{h}_{0}(\xi)} \leqslant \sqrt{\gamma}+2 \gamma
$$

would lead to a contradiction. We claim that there exists a constant $\gamma(\bar{M})$ depending only on $\bar{M}$ such that, for all $\gamma \leqslant \gamma(\bar{M}), \xi \in \mathbb{R}$, and $t \in[0, T]$,

$$
\frac{\tilde{q}}{\tilde{q}+\overline{\tilde{h}}}(t, \xi) \leqslant \gamma \quad \text { and } \quad \tilde{w}(t, \xi)=0 \quad \text { implies } \tilde{q}(t, \xi)=0,
$$

and

$$
\frac{\tilde{q}}{\tilde{q}+\overline{\tilde{h}}}(t, \xi) \leqslant \gamma \quad \text { implies }\left(\frac{\tilde{w}}{\tilde{q}+\overline{\tilde{h}}}\right)_{t}(t, \xi) \geqslant 0 .
$$

We consider a fixed $\xi \in \mathbb{R}$, and suppress it in the notation. If $\tilde{w}(t)=0$, then (59) yields $\tilde{q}(t) \overline{\tilde{h}}(t)=0$. Thus, either $\tilde{q}(t)=0$ or $\overline{\tilde{h}}(t)=0$. Assume that $\tilde{q}(t) \neq 0$; then $\overline{\tilde{h}}(t)=0$. Hence, $1-\gamma \leqslant \overline{\tilde{h}}(t) /(\tilde{q}(t)+\overline{\tilde{h}}(t))=0$, and we are led to a contradiction. Hence, $\tilde{q}(t)=0$, and we have proved (67).

If $(\tilde{q} /(\tilde{q}+\overline{\tilde{h}}))(t) \leqslant \gamma$, we have

$$
\begin{aligned}
\left(\frac{\tilde{w}}{\tilde{q}+\overline{\tilde{h}}}\right)_{t} & =\frac{1}{2}+\left(U^{2}-P(\Theta)-\frac{1}{2}\right) \frac{\tilde{q}}{\tilde{q}+\overline{\tilde{h}}}-\left(2 U^{2}-2 P(\Theta)+1\right) \frac{\tilde{w}^{2}}{(\tilde{q}+\overline{\tilde{h}})^{2}} \\
& \geqslant \frac{1}{2}-C(\bar{M}) \frac{\tilde{q}}{\tilde{q}+\overline{\tilde{h}}}-C(\bar{M}) \frac{\tilde{\tilde{q}}}{(\tilde{\tilde{q}}+\overline{\tilde{h}})^{2}} \\
& \geqslant \frac{1}{2}-C(\bar{M}) \gamma .
\end{aligned}
$$

Recall that we allow for a redefinition of $C(\bar{M})$. By choosing $\gamma(\bar{M}) \leqslant$ $(4 C(\bar{M}))^{-1}$, we get $(\tilde{w} /(\tilde{q}+\overline{\tilde{h}}))_{t} \geqslant 0$, and we have proved (68). For any $\gamma \leqslant \gamma(\bar{M})$, we consider a given $\xi$ in $\kappa_{1-\gamma}$, and again suppress it in the notation. We define

$$
t_{0}=\sup \left\{\begin{array}{l|l}
t \in\left[0, \tilde{\tau}_{1}\right] & \frac{\tilde{q}}{\tilde{q}+\overline{\tilde{h}}}\left(t^{\prime}\right)<2 \gamma \text { and } \tilde{w}\left(t^{\prime}\right)<0 \text { for all } t^{\prime} \leqslant t
\end{array}\right\} .
$$

Let us prove that $t_{0}=\tilde{\tau}_{1}$. Assume the opposite; that is, $t_{0}<\tilde{\tau}_{1}$. Then we have either $(\tilde{q} /(\tilde{q}+\overline{\tilde{h}}))\left(t_{0}\right)=2 \gamma$ or $\tilde{w}\left(t_{0}\right)=0$. We have $((\tilde{q} /(\tilde{q}+\overline{\tilde{h}})))_{t} \leqslant 0$ on $[0$, $\left.t_{0}\right]$, and $(\tilde{q} /(\tilde{q}+\tilde{\tilde{h}}))(t)$ is decreasing on this interval. Hence, $(\tilde{q} /(\tilde{q}+\overline{\tilde{h}}))\left(t_{0}\right) \leqslant$ 
$(\tilde{q} /(\tilde{q}+\overline{\tilde{h}}))(0) \leqslant \gamma$, and therefore we must have $\tilde{w}\left(t_{0}\right)=0$. Then (67) implies that $\tilde{q}\left(t_{0}\right)=0$, and therefore $t_{0}=\tilde{\tau}_{1}$, which contradicts our assumption. From (69), we get, for $\gamma$ sufficiently small,

$$
0=\frac{\tilde{w}}{\tilde{q}+\overline{\tilde{h}}}\left(\tilde{\tau}_{1}-0\right) \geqslant \frac{\tilde{w}}{\tilde{q}+\overline{\tilde{h}}}(0)+\frac{1}{4} \tilde{\tau}_{1},
$$

and therefore $\tilde{\tau}_{1} \leqslant 4 \sqrt{\gamma}$. By taking $\gamma$ small enough, we can impose $\tilde{\tau}_{1}<T$, which proves (63). It is clear from $(68)$ that $\tilde{w} /(\tilde{q}+\overline{\tilde{h}})$ is increasing. Assume that $\tilde{\Theta}(t, \xi)$ leaves $\Omega_{1}$ for some $t<\min \left(\tilde{\tau}_{1}, T\right)$. Then we get

$$
1=\frac{\tilde{q}(t)+\overline{\tilde{h}}(t)}{\tilde{q}(t)+\overline{\tilde{h}}(t)} \leqslant \frac{|\tilde{w}(t)|+2 \tilde{q}(t)}{\tilde{q}(t)+\overline{\tilde{h}}(t)} \leqslant \sqrt{\gamma}+2 \gamma,
$$

and, by taking $\gamma$ small enough, we are led to a contradiction.

(iv) Without loss of generality, we assume that $\hat{T} \leqslant 1$. From (iii), we know that there exists a $\gamma^{\prime}$ depending only on $\bar{M}$ such that, for $\xi \in \kappa_{1-\gamma^{\prime}}$, we have that $\tilde{q} /(\tilde{q}+\overline{\tilde{h}})$ is a decreasing function, and $\tilde{w} /(\tilde{q}+\overline{\tilde{h}})$ is an increasing function, both with respect to time, on $\left[0, \min \left(\tilde{\tau}_{1}, T\right)\right]$. Let $\bar{\gamma} \leqslant \min \left(\gamma, \gamma^{\prime}\right)$. We consider a fixed $\xi \in \mathbb{R}$ such that $\tilde{\tau}_{1}(\xi)<\hat{T}$ (which means implicitly that $\tilde{r}(t, \xi)=0$ for all $t$ ), but $\xi \notin \kappa_{1-\bar{\gamma}}$. We will suppress $\xi$ in the notation from now on. Let us introduce

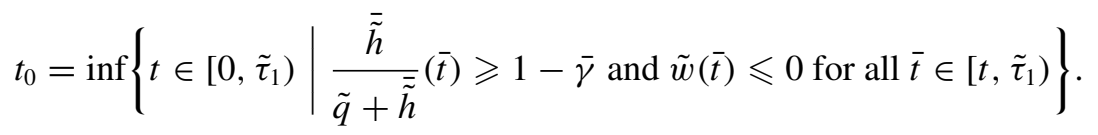

Since $\tilde{w}_{t}\left(\tilde{\tau}_{1}\right)=\frac{1}{2} \overline{\tilde{h}}\left(\tilde{\tau}_{1}\right) \geqslant 0$ and $\tilde{w}\left(\tilde{\tau}_{1}\right)=\tilde{q}\left(\tilde{\tau}_{1}\right)=0$, the definition of $t_{0}$ is well posed when $\tilde{\tau}_{1}>0$, and we have $t_{0}<\tilde{\tau}_{1}$. By assumption, $t_{0}>0$, and $\tilde{w}\left(t_{0}\right)=0$ or $(\overline{\tilde{h}} /(\tilde{q}+\overline{\tilde{h}}))\left(t_{0}\right)=1-\bar{\gamma}$. We cannot have $\tilde{w}\left(t_{0}\right)=0$, since it would imply, see (67), that $\tilde{q}\left(t_{0}\right)=0$ and therefore $t_{0}=\tilde{\tau}_{1}$, which is not possible. Thus we must have $(\overline{\tilde{h}} /(\tilde{q}+\overline{\tilde{h}}))\left(t_{0}\right)=1-\bar{\gamma}$, and, in particular, $(\tilde{q} /(\tilde{q}+\overline{\tilde{h}}))\left(t_{0}\right)=\bar{\gamma}$. According to the choice of $\bar{\gamma}$, we have that $(\tilde{q} /(\tilde{q}+\overline{\tilde{h}}))(t) \leqslant \bar{\gamma}$ for all $t \geqslant t_{0}$, and $(\tilde{w} /(\tilde{q}+\overline{\tilde{h}}))(t)$ is increasing. Then we have, following the same lines as in (69),

$$
\left(\frac{\tilde{w}}{\tilde{q}+\overline{\tilde{h}}}\right)_{t} \geqslant \frac{1}{2}-C(\bar{M}) \bar{\gamma},
$$

which yields for $0 \leqslant t_{0} \leqslant t^{\prime} \leqslant \min \left(\tilde{\tau}_{1}, 1\right)$ that

$$
\frac{\tilde{w}}{\tilde{q}+\overline{\tilde{h}}}\left(t^{\prime}\right) \geqslant \frac{\tilde{w}}{\tilde{q}+\overline{\tilde{h}}}\left(t_{0}\right)+\left(t^{\prime}-t_{0}\right)\left(\frac{1}{2}-C(\bar{M}) \bar{\gamma}\right) .
$$


Since $\tilde{w} /(\tilde{q}+\overline{\tilde{h}})\left(t_{0}\right)=-\sqrt{\bar{\gamma}(1-\underline{\bar{\gamma}})}$, we choose $\hat{T}$ such that $0>-\sqrt{\bar{\gamma}(1-\bar{\gamma})}+$ $\hat{T}\left(\frac{1}{2}-C(\bar{M}) \bar{\gamma}\right)$. Thus $(\tilde{w} /(\tilde{q}+\overline{\tilde{h}}))(\hat{T})<0$, and therefore all points which experience wave breaking before $\hat{T}$ are contained in $\kappa_{1-\bar{\gamma}}$, since any point entering $\kappa_{1-\bar{\gamma}}$ at a later time cannot reach the origin within the time interval $[0, \hat{T}]$ according to the last estimate.

LEMMA 11. Given $M>0$, there exist $\bar{T}$ and $\bar{M}$ such that, for all $T \leqslant \bar{T}$ and any initial data $\Theta_{0} \in \mathcal{G} \cap B_{M}, \mathcal{P}$ is a mapping from $C\left([0, T], B_{\bar{M}}\right)$ to $C\left([0, T], B_{\bar{M}}\right)$.

Proof. To simplify the notation, we will generically denote by $K(M)$ and $C(\bar{M})$ increasing functions of $M$ and $\bar{M}$, respectively. Without loss of generality, we assume that $\bar{T} \leqslant 1$.

Let $\Theta \in C\left([0, T], B_{\bar{M}}\right)$ for a value of $\bar{M}$ that will be determined at the end as a function of $M$. We assume without loss of generality that $\bar{M} \geqslant M$. Let $\tilde{\Theta}=$ $\mathcal{P}(\Theta)$. From Lemma 8, we have

$$
\|Q(\Theta)\|_{L_{T}^{\infty} E} \leqslant C(\bar{M}), \quad\|P(\Theta)\|_{L_{T}^{\infty} E} \leqslant C(\bar{M}) .
$$

Since $\tilde{U}_{t}=-Q(\Theta)$, we get

$$
\|\tilde{U}\|_{L_{T}^{\infty} E} \leqslant\left\|U_{0}\right\|_{E}+T\|Q(\Theta)\|_{L_{T}^{\infty} E} \leqslant M+T C(\bar{M}) .
$$

Similarly, since, $\tilde{\zeta}_{t}=\tilde{U}$, we get

$$
\|\tilde{\zeta}\|_{L_{T}^{\infty} L^{\infty}} \leqslant\left\|\zeta_{0}\right\|_{L^{\infty}}+T\|\tilde{U}\|_{L_{T}^{\infty} L^{\infty}} \leqslant M+T C(\bar{M}) .
$$

From (54), by the Minkowski inequality for integrals, we get

$$
\begin{aligned}
\|\tilde{v}(t, \cdot)\|_{E} \leqslant & \left\|v_{0}\right\|_{E}+\int_{0}^{t}\left\|\tilde{w}\left(t^{\prime}, \cdot\right)\right\|_{E} d t^{\prime} \\
\|\tilde{w}(t, \cdot)\|_{E} \leqslant & \left\|w_{0}\right\|_{E}+T\left\|P(\Theta)-U^{2}\right\|_{L_{T}^{\infty} E} \\
& \quad+\int_{0}^{t}\left(\frac{1}{2}\left\|\tilde{h}\left(t^{\prime}, \cdot\right)\right\|_{E}+\left\|U^{2}-P(\Theta)\right\|_{L_{T}^{\infty} E}\left\|\tilde{v}\left(t^{\prime}, \cdot\right)\right\|_{E}\right) d t^{\prime} \\
\|\tilde{h}(t, \cdot)\|_{E} \leqslant & \left\|h_{0}\right\|_{E}+2 \int_{0}^{t}\left\|U^{2}-P(\Theta)\right\|_{L_{T}^{\infty} E}\left\|\tilde{w}\left(t^{\prime}, \cdot\right)\right\|_{E} d t^{\prime} \\
\|\tilde{r}(t, \cdot)\|_{E} \leqslant & \left\|r_{0}\right\|_{E} .
\end{aligned}
$$

Here, we used that $0 \leqslant \overline{\tilde{h}}(t, \xi) \leqslant \tilde{h}(t, \xi)$ and that $\tilde{h}(t, \xi)$ is continuous with respect to time. These inequalities imply that

$$
\|\tilde{Z}(t, \cdot)\|_{\bar{W}} \leqslant K(M)+T C(\bar{M})+C(\bar{M}) \int_{0}^{t}\left\|\tilde{Z}\left(t^{\prime}, \cdot\right)\right\|_{E} d t^{\prime},
$$


and, applying Gronwall's inequality,

$$
\|\tilde{Z}\|_{L_{T}^{\infty} \bar{W}} \leqslant(K(M)+T C(\bar{M})) e^{C(\bar{M}) T} .
$$

Gathering (72), (73), and (76), we get

$$
\|\tilde{X}\|_{L_{T}^{\infty} \bar{V}} \leqslant(K(M)+T C(\bar{M})) e^{C(\bar{M}) T} .
$$

Moreover, (54) implies that

$$
\|\tilde{h}(t, \cdot)\|_{L^{1}} \leqslant\left\|\tilde{h}_{0}\right\|_{L^{1}}+2 T\left\|U^{2}-P(\Theta)\right\|_{L_{T}^{\infty} L^{2}}\|\tilde{w}\|_{L_{T}^{\infty} L^{2}},
$$

and hence

$$
\|\tilde{h}\|_{L_{T}^{\infty} L^{1}} \leqslant(K(M)+T C(\bar{M})) e^{C(\bar{M}) T} .
$$

From (60), we get

$$
\left\|\frac{1}{\tilde{q}+\tilde{h}}\right\|_{L_{T}^{\infty} L^{\infty}} \leqslant K(M) e^{C(\bar{M}) T} .
$$

Thus, we finally obtain

$$
\|\tilde{X}\|_{L_{T}^{\infty} \bar{V}}+\|\tilde{h}\|_{L_{T}^{\infty} L^{1}}+\left\|\frac{1}{\tilde{q}+\tilde{h}}\right\|_{L_{T}^{\infty} L^{\infty}} \leqslant(K(M)+T C(\bar{M})) e^{C(\bar{M}) T}
$$

for some constants $K(M)$ and $C(\bar{M})$ that depend only on $M$ and $\bar{M}$, respectively. We now set $\bar{M}=2 K(M)$. Then we can choose $T$ so small that $(K(M)+$ $C(\bar{M}) T) e^{C(\bar{M}) T} \leqslant 2 K(M)=\bar{M}$, and therefore

$$
\|\tilde{X}\|_{L_{T}^{\infty} \bar{V}}+\|\tilde{h}\|_{L_{T}^{\infty} L^{1}}+\left\|\frac{1}{\tilde{q}+\tilde{h}}\right\|_{L_{T}^{\infty} L^{\infty}} \leqslant \bar{M} .
$$

Given $\Theta_{0} \in \mathcal{G} \cap B_{M}$, there exists $\bar{M}$, which depends only on $M$, such that $\mathcal{P}$ is a mapping from $C\left([0, T], B_{\bar{M}}\right)$ to $C\left([0, T], B_{\bar{M}}\right)$ for $T$ small enough. Therefore, we set

$$
\operatorname{Im}(\mathcal{P})=\left\{\mathcal{P}(\Theta) \mid \Theta \in C\left([0, T], B_{\bar{M}}\right)\right\}
$$

LEMMA 12. Given $\Theta_{0} \in \mathcal{G} \cap B_{M}$, given $\Theta \in C\left([0, T], B_{\bar{M}}\right)$, we denote $\tilde{\Theta}=$ $\mathcal{P}(\Theta) \in C\left([0, T], B_{\bar{M}}\right)$ with initial data $\left.\tilde{\Theta}\right|_{t=0}=\Theta_{0}$.

Then there exists a time $\hat{T}$ depending on $\bar{M}$ such that any point $\xi$ can experience wave breaking at most once within the time interval $\left[T_{0}, T_{0}+\hat{T}\right]$ for any $T_{0} \geqslant 0$. More precisely, given $\xi \in \mathbb{R}$, we have

$$
\tilde{\tau}_{j+1}(\xi)-\tilde{\tau}_{j}(\xi)>\hat{T} \quad \text { for all } j .
$$

In addition, for $\hat{T}$ sufficiently small, we get that in this case $\tilde{w}(t, \xi) \geqslant 0$ for all $t \in\left[\tilde{\tau}_{j}(\xi), \tilde{\tau}_{j}(\xi)+\hat{T}\right]$. 
Proof. If no wave breaking occurs within $[0, T]$ or $\alpha=1$, there is nothing to prove. Therefore, let us assume that $\alpha \in[0,1)$, and that for some fixed $\xi \in \mathbb{R}$ wave breaking occurs. Moreover, let us assume the worst possible case, namely $T_{0}=\tilde{\tau}_{1}(\xi)$, since all other cases follow from this one. At time $\tilde{\tau}_{1}(\xi)$, we have $\tilde{q}\left(\tilde{\tau}_{1}(\xi), \xi\right)=\tilde{w}\left(\tilde{\tau}_{1}(\xi), \xi\right)=0$, and, in particular, $(\overline{\tilde{h}} /(\tilde{q}+\overline{\tilde{h}}))\left(\tilde{\tau}_{1}(\xi), \xi\right)=1$ and $\tilde{r}(t, \xi)=0$ for all $t$. Moreover, wave breaking can only take place if $\tilde{w}(t, \xi) \leqslant 0$ for $\tilde{\tau}_{1}(\xi)-\varepsilon \leqslant t \leqslant \tilde{\tau}_{1}(\xi)$, but right after wave breaking $\tilde{w}(t, \xi)$ is positive, in the case where $\alpha<1$. Thus, before wave breaking can occur once more at $\xi \in \mathbb{R}$, $\tilde{w}(t, \xi)$ has to change sign from positive to negative at some time $t^{*}>\tilde{\tau}_{1}(\xi)$. Hence, we will now establish a lower bound on $t^{*}-\tilde{\tau}_{1}(\xi)$, which defines $\hat{T}$.

Let $t^{*}$ be the first time after the first collision where $\tilde{w}(t, \xi)$ changes sign. We have $t^{*}>\tilde{\tau}_{1}(\xi)$, and (59) implies that either $\tilde{q}\left(t^{*}, \xi\right)=0$ (that is, wave breaking occurs) or $(\overline{\tilde{h}} /(\tilde{q}+\overline{\tilde{h}}))\left(t^{*}, \xi\right)=0$ (that is, no wave breaking occurs). The first alternative is not possible, as $\tilde{q}_{t}(t, \xi)=\tilde{w}(t, \xi)>0$ for $t \in\left(\tau_{1}(\xi), t^{*}\right)$, in the case where $\alpha<1$. Hence, $\tilde{q}\left(t^{*}, \xi\right) /\left(\tilde{q}\left(t^{*}, \xi\right)+\overline{\tilde{h}}\left(t^{*}, \xi\right)\right)=1$. Thus, if we can establish a lower bound on how long it takes for the function $\tilde{q}(t, \xi) /(\tilde{q}(t, \xi)+\overline{\tilde{h}}(t, \xi))$, which equals 0 at time $\tilde{\tau}_{1}(\xi)$, to reach 1 after wave breaking, the claim follows.

Observe first that (59) implies that

$$
\left|\frac{\tilde{w}}{\tilde{q}+\overline{\tilde{h}}}(t, \xi)\right| \leqslant \frac{1}{\sqrt{2}}, \quad \text { and } \quad\left|\frac{\tilde{r}}{\tilde{q}+\overline{\tilde{h}}}(t, \xi)\right| \leqslant \frac{1}{\sqrt{2}}
$$

for all $t \in[0, \infty)$ and $\xi \in \mathbb{R}$. Moreover, according to Lemma 8(i), we have

$$
\|P(\Theta)\|_{L_{T}^{\infty} L^{\infty}}+\|U\|_{L_{T}^{\infty} L^{\infty}}^{2} \leqslant C(\bar{M}) .
$$

From (54), we get

$$
\begin{aligned}
\left(\frac{\tilde{q}}{\tilde{q}+\overline{\tilde{h}}}\right)_{t} & =\frac{\tilde{w}}{\tilde{q}+\overline{\tilde{h}}}\left(1-2 \frac{\tilde{q}}{\tilde{q}+\overline{\tilde{h}}}\left(U^{2}-P(\Theta)+\frac{1}{2}\right)\right) \\
& \leqslant \frac{1}{\sqrt{2}}(1+C(\bar{M})),
\end{aligned}
$$

for $t \in\left[\tilde{\tau}_{1}(\xi), \tilde{\tau}_{2}(\xi)\right)$. Hence, integrating the latter equation in time from $\tau_{1}$ to $t^{*}$ yields $1=(\tilde{q} /(\tilde{q}+\tilde{\tilde{h}}))\left(t^{*}\right) \leqslant \frac{1}{\sqrt{2}}\left(t^{*}-\tau_{1}(\xi)\right)(1+C(\bar{M}))$. Choosing $\hat{T}=\sqrt{2}(1+$ $C(\bar{M}))^{-1}$ concludes the proof.

We define the discontinuity residual as

$$
\Gamma(\Theta, \tilde{\Theta})=\int_{0}^{T} \int_{\mathbb{R}}|\bar{h}(t, \xi)-\overline{\tilde{h}}(t, \xi)| d \xi d t .
$$


According to Lemma 8(ii), we have

$$
\begin{aligned}
& \|Q(\Theta)-Q(\tilde{\Theta})\|_{L_{T}^{1} E}+\|P(\Theta)-P(\tilde{\Theta})\|_{L_{T}^{1} E} \\
& \leqslant C(\bar{M})\left(T\|X-\tilde{X}\|_{L_{T}^{\infty} \bar{V}}+\Gamma(\Theta, \tilde{\Theta})\right) .
\end{aligned}
$$

In the next lemma, we establish some estimates for $\Gamma(\Theta, \tilde{\Theta}), \Gamma(\mathcal{P}(\Theta)$, $\mathcal{P}(\tilde{\Theta}))$, and a quasicontraction property for $\mathcal{P}$.

LEMma 13. Given $\Theta, \tilde{\Theta} \in \operatorname{Im}(\mathcal{P})$, and $\gamma \in\left(0, \frac{1}{2}\right)$, let $\Theta_{2}=\mathcal{P}(\Theta)$ and $\tilde{\Theta}_{2}=\mathcal{P}(\tilde{\Theta})$; then there exists $T>0$ depending on $\bar{M}$ such that the following inequalities hold.

(i)

$$
\left\|h_{2}-\tilde{h}_{2}\right\|_{L_{T}^{\infty} L^{1}} \leqslant C(\bar{M}) T\left(\left\|X_{2}-\tilde{X}_{2}\right\|_{L_{T}^{\infty} \bar{V}}+\|X-\tilde{X}\|_{L_{T}^{\infty} \bar{V}}+\Gamma(\Theta, \tilde{\Theta})\right),
$$

(ii)

$$
\Gamma(\Theta, \tilde{\Theta}) \leqslant C(\bar{M})\|X-\tilde{X}\|_{L_{T}^{\infty} \bar{V}}
$$

(iii)

$$
\Gamma\left(\Theta_{2}, \tilde{\Theta}_{2}\right) \leqslant C(\bar{M})\left(T\left(\left\|X_{2}-\tilde{X}_{2}\right\|_{L_{T}^{\infty} \bar{V}}+\|X-\tilde{X}\|_{L_{T}^{\infty} \bar{V}}\right)+\gamma \Gamma(\Theta, \tilde{\Theta})\right),
$$

$$
\left\|X_{2}-\tilde{X}_{2}\right\|_{L_{T}^{\infty} \bar{V}} \leqslant C(\bar{M})\left(T\|X-\tilde{X}\|_{L_{T}^{\infty} \bar{V}}+\Gamma(\Theta, \tilde{\Theta})\right),
$$

where $C(\bar{M})$ denotes some constant which depends only on $\bar{M}$.

Proof. Denote $\Theta_{2}=\mathcal{P}(\Theta)$ and $\tilde{\Theta}_{2}=\mathcal{P}(\tilde{\Theta})$, and, abusing the notation, let $\tau_{2}(\xi)$ and $\tilde{\tau}_{2}(\xi)$ be the first time when wave breaking occurs at the point $\xi \in \mathbb{R}$ for $\Theta_{2}$ and $\tilde{\Theta}_{2}$, respectively. Given $\gamma>0$, we know from Lemma 10(iv) and Lemma 12 that there exists $T$ small enough such that $\left\{\xi \in \mathbb{R} \mid 0<\tau_{2}(\xi)<T\right.$ or $0<\tilde{\tau}_{2}(\xi)<$ $T\} \subset \kappa_{1-\gamma}$ and such that every point experiences wave breaking at most once within the time interval $[0, T]$. We consider such $T$. Without loss of generality, we can assume that $T \leqslant 1$ and $\gamma \leqslant \gamma(\bar{M})$.

(i) From (54), we get

$$
\begin{aligned}
\| h_{2} & -\tilde{h}_{2} \|_{L_{T}^{\infty} L^{1}} \\
\leqslant & \int_{0}^{T}\left\|2\left(U^{2}-P(\Theta)\right)\left(w_{2}-\tilde{w}_{2}\right)(s, \cdot)\right\|_{L^{1}} d s \\
& \quad+\int_{0}^{T}\left\|2\left(U^{2}-P(\Theta)-\tilde{U}^{2}+P(\tilde{\Theta})\right) \tilde{w}_{2}(s, \cdot)\right\|_{L^{1}} d s
\end{aligned}
$$




$$
\begin{aligned}
\leqslant & \int_{0}^{T}\left\|2\left(U^{2}-P(\Theta)\right)(s, \cdot)\right\|_{L^{2}}\left\|\left(w_{2}-\tilde{w}_{2}\right)(s, \cdot)\right\|_{L^{2}} d s \\
& +\int_{0}^{T} 2\left(\left\|\left(U^{2}-\tilde{U}^{2}\right)(s, \cdot)\right\|_{L^{2}}+\|(P(\Theta)-P(\tilde{\Theta}))(s, \cdot)\|_{L^{2}}\right)\left\|\tilde{w}_{2}(s, \cdot)\right\|_{L^{2}} d s \\
\leqslant & C(\bar{M}) T\left(\left\|X_{2}-\tilde{X}_{2}\right\|_{L_{T}^{\infty} \bar{V}}+\|X-\tilde{X}\|_{L_{T}^{\infty} \bar{V}}+\|P(\Theta)-P(\tilde{\Theta})\|_{L_{T}^{\infty} E}\right) \\
\leqslant & C(\bar{M}) T\left(\left\|X_{2}-\tilde{X}_{2}\right\|_{L_{T}^{\infty} \bar{V}}+\|X-\tilde{X}\|_{L_{T}^{\infty} \bar{V}}+\Gamma(\Theta, \tilde{\Theta})\right) .
\end{aligned}
$$

As far as the other estimates are concerned, observe first that for $\xi \in \kappa_{1-\gamma}^{c}$ no wave breaking occurs, and therefore $\left|\bar{h}_{2}(t, \xi)-\overline{\tilde{h}}_{2}(t, \xi)\right|=\mid h_{2}(t, \xi)-\tilde{h}_{2}(t$, $\xi) \mid$, since $\Theta_{2}(0, \xi)=\tilde{\Theta}_{2}(0, \xi)$. Moreover, using (59), we get $\bar{h}_{2}(t, \xi)=w_{2}^{2}(t$, $\xi)+r_{2}^{2}(t, \xi)-\bar{h}_{2}(t, \xi) v_{2}(t, \xi)$, and a similar relation holds for $\overline{\tilde{h}}_{2}(t, \xi)$. Hence

$$
\begin{aligned}
\int_{0}^{T} & \int_{\kappa_{1-\gamma}^{c}}\left|\bar{h}_{2}(t, \xi)-\overline{\tilde{h}}_{2}(t, \xi)\right| d \xi d t \\
\leqslant & \int_{0}^{T} \int_{\kappa_{1-\gamma}^{c}}\left|\left(w_{2}+\tilde{w}_{2}\right)\left(w_{2}-\tilde{w}_{2}\right)\right|(t, \xi) d \xi d t \\
& +\int_{0}^{T} \int_{\kappa_{1-\gamma}^{c}}\left|\left(r_{2}+\tilde{r}_{2}\right)\left(r_{2}-\tilde{r}_{2}\right)\right|(t, \xi) d \xi d t \\
& +\int_{0}^{T} \int_{\kappa_{1-\gamma}^{c}}\left(\left|\left(\bar{h}_{2}-\overline{\tilde{h}}_{2}\right) v_{2}\right|(t, \xi)+\overline{\tilde{h}}_{2}\left|v_{2}-\tilde{v}_{2}\right|(t, \xi)\right) d \xi d t \\
\leqslant & C(\bar{M}) T\left\|X_{2}-\tilde{X}_{2}\right\|_{L_{T}^{\infty} \bar{V}},
\end{aligned}
$$

where we used the Cauchy-Schwarz inequality in the last step. Thus we have

$$
\begin{aligned}
& \int_{0}^{T} \int_{\mathbb{R}}\left|\bar{h}_{2}(t, \xi)-\overline{\tilde{h}}_{2}(t, \xi)\right| d \xi d t \\
& \quad \leqslant C(\bar{M}) T\left\|X_{2}-\tilde{X}_{2}\right\|_{L_{T}^{\infty} \bar{V}}+\int_{0}^{T} \int_{\kappa_{1-\gamma}}\left|\bar{h}_{2}(t, \xi)-\overline{\tilde{h}}_{2}(t, \xi)\right| d \xi d t .
\end{aligned}
$$

(ii) Let us consider $\xi \in \kappa_{1-\gamma}$ such that $\tau_{2}(\xi) \neq \tilde{\tau}_{2}(\xi)$. Without loss of generality, we assume that $0<\tau_{2}(\xi)<\tilde{\tau}_{2}(\xi) \leqslant T$. Since $\Theta_{2}(t, \xi)$ and $\tilde{\Theta}_{2}(t, \xi)$ both belong to $\operatorname{Im}(\mathcal{P})$, we have that $\left|h_{2}(t, \xi)-\tilde{h}_{2}(t, \xi)\right|=\left|\bar{h}_{2}(t, \xi)-\overline{\tilde{h}}_{2}(t, \xi)\right|$ for $t \in[0$, $\left.\tau_{2}(\xi)\right)$, and especially

$$
\int_{0}^{\tau_{2}}\left|\bar{h}_{2}(t, \xi)-\overline{\tilde{h}}_{2}(t, \xi)\right| d t=\int_{0}^{\tau_{2}}\left|h_{2}(t, \xi)-\tilde{h}_{2}(t, \xi)\right| d t .
$$


For $t \in\left[\tau_{2}(\xi), \tilde{\tau}_{2}(\xi)\right)$, we have $\bar{h}_{2}(t, \xi)=h_{2}(t, \xi)-l_{0}(\xi)-l_{1}(\xi)$ and $\overline{\tilde{h}}_{2}(t$, $\xi)=\tilde{h}_{2}(t, \xi)-l_{0}(\xi)$. Hence, it follows that

$$
\left|\bar{h}_{2}(t, \xi)-\overline{\tilde{h}}_{2}(t, \xi)\right| \leqslant\left|h_{2}(t, \xi)-\tilde{h}_{2}(t, \xi)\right|+l_{1}(\xi) .
$$

Since (58) implies that $0 \leqslant l_{1}(\xi) \leqslant h_{2}(t, \xi)-l_{0}(\xi)$ for all $t \in\left[\tau_{2}(\xi), \tilde{\tau}_{2}(\xi)\right]$, we get

$$
\begin{aligned}
\int_{\tau_{2}}^{\tilde{\tau}_{2}} l_{1}(\xi) d t & \leqslant \int_{\tau_{2}}^{\tilde{\tau}_{2}}\left(h_{2}(t, \xi)-l_{0}(\xi)\right) d t \\
& \leqslant \int_{\tau_{2}}^{\tilde{\tau}_{2}}\left|h_{2}(t, \xi)-\tilde{h}_{2}(t, \xi)\right| d t+\int_{\tau_{2}}^{\tilde{\tau}_{2}}\left(\tilde{h}_{2}(t, \xi)-l_{0}(\xi)\right) d t \\
& \leqslant \int_{\tau_{2}}^{\tilde{\tau}_{2}}\left|h_{2}(t, \xi)-\tilde{h}_{2}(t, \xi)\right| d t+\int_{\tau_{2}}^{\tilde{\tau}_{2}} \tilde{\tilde{h}}_{2}(t, \xi) d t
\end{aligned}
$$

Since $\tilde{\Theta}_{2}=\mathcal{P}(\tilde{\Theta})$ for $\tilde{\Theta} \in C\left([0, T], B_{\bar{M}}\right)$, we get, using (54), for $t \in\left[\tau_{2}(\xi)\right.$, $\tilde{\tau}_{2}(\xi)$ ], that

$$
\tilde{w}_{2}(t, \xi)=\tilde{w}_{2}\left(\tau_{2}(\xi), \xi\right)+\frac{1}{2} \int_{\tau_{2}}^{t} \overline{\tilde{h}}_{2}\left(t^{\prime}, \xi\right) d t^{\prime}+\int_{\tau_{2}}^{t}\left(\tilde{U}^{2}-P(\tilde{\Theta})\right) \tilde{q}_{2}\left(t^{\prime}, \xi\right) d t^{\prime}
$$

According to Lemma 10 , since $\xi \in \kappa_{1-\gamma}$, we have $\tilde{\Theta}_{2}(t, \xi) \in \Omega_{1}$ for all $t \in[0$, $\left.\min \left(\tilde{\tau}_{2}(\xi), T\right)\right]$. Moreover, $\tilde{w}_{2}(t, \xi) \leqslant 0$ on the interval $\left[0, \min \left(\tilde{\tau}_{2}(\xi), T\right)\right]$, while $\tilde{q}_{2}(t, \xi)$ is decaying. Furthermore, $\left\|\tilde{U}^{2}-P(\tilde{\Theta})\right\|_{L_{T}^{\infty} E} \leqslant C(\bar{M}), w_{2}\left(\tau_{2}(\xi), \xi\right)=$ $q_{2}\left(\tau_{2}(\xi), \xi\right)=0$, and $w_{2}(t, \xi) \geqslant 0$ for all $t \in\left[\tau_{2}(\xi), T\right]$. Thus we get that

$$
\begin{aligned}
& \frac{1}{2} \int_{\tau_{2}}^{\tilde{\tau}_{2}} \overline{\tilde{h}}_{2}\left(t^{\prime}, \xi\right) d t^{\prime} \\
& \quad=\tilde{w}_{2}\left(\tilde{\tau}_{2}(\xi), \xi\right)-\tilde{w}_{2}\left(\tau_{2}(\xi), \xi\right)-\int_{\tau_{2}}^{\tilde{\tau}_{2}}\left(\tilde{U}^{2}-P(\tilde{\Theta})\right) \tilde{q}_{2}\left(t^{\prime}, \xi\right) d t^{\prime} \\
& \quad \leqslant-\tilde{w}_{2}\left(\tau_{2}(\xi), \xi\right)+C(\bar{M}) \int_{\tau_{2}}^{\tilde{\tau}_{2}} \tilde{q}_{2}\left(\tau_{2}(\xi), \xi\right) d t^{\prime} \\
& \quad \leqslant w_{2}\left(\tau_{2}(\xi), \xi\right)-\tilde{w}_{2}\left(\tau_{2}(\xi), \xi\right)+C(\bar{M}) \int_{\tau_{2}}^{\tilde{\tau}_{2}} \tilde{q}_{2}\left(\tau_{2}(\xi), \xi\right)-q_{2}\left(\tau_{2}(\xi), \xi\right) d t^{\prime} \\
& \quad \leqslant w_{2}\left(\tau_{2}(\xi), \xi\right)-\tilde{w}_{2}\left(\tau_{2}(\xi), \xi\right)+C(\bar{M}) T\left\|X_{2}(\cdot, \xi)-\tilde{X}_{2}(\cdot, \xi)\right\|_{L_{\tau_{2}}^{\infty}} \\
& \quad \leqslant w_{2}\left(\tau_{2}(\xi), \xi\right)-\tilde{w}_{2}\left(\tau_{2}(\xi), \xi\right)+C(\bar{M}) T\left\|X_{2}-\tilde{X}_{2}\right\|_{L_{T}^{\infty} \bar{V}}
\end{aligned}
$$


Combining the above estimates yields

$$
\begin{aligned}
& \int_{\tau_{2}}^{\tilde{\tau}_{2}}\left|\bar{h}_{2}(t, \xi)-\overline{\tilde{h}}_{2}(t, \xi)\right| d t \leqslant \int_{\tau_{2}}^{\tilde{\tau}_{2}}\left|h_{2}(t, \xi)-\tilde{h}_{2}(t, \xi)\right| d t+\int_{\tau_{2}}^{\tilde{\tau}_{2}} l_{1}(\xi) d t \\
& \leqslant 2 \int_{\tau_{2}}^{\tilde{\tau}_{2}}\left|h_{2}(t, \xi)-\tilde{h}_{2}(t, \xi)\right| d t+\int_{\tau_{2}}^{\tilde{\tau}_{2}} \overline{\tilde{h}}_{2}(t, \xi) d t \\
& \leqslant 2\left(w_{2}\left(\tau_{2}(\xi), \xi\right)-\tilde{w}_{2}\left(\tau_{2}(\xi), \xi\right)\right) \\
& \quad+2 \int_{\tau_{2}}^{\tilde{\tau}_{2}}\left|h_{2}(t, \xi)-\tilde{h}_{2}(t, \xi)\right| d t+C(\bar{M}) T\left\|X_{2}-\tilde{X}_{2}\right\|_{L_{T}^{\infty} \bar{V}}
\end{aligned}
$$

For $t \in\left[\tilde{\tau}_{2}(\xi), T\right]$, we have $\bar{h}_{2}(t, \xi)=h_{2}(t, \xi)-l_{0}(\xi)-l_{1}(\xi)$ and $\overline{\tilde{h}}_{2}(t, \xi)=$ $\tilde{h}_{2}(t, \xi)-l_{0}(\xi)-\tilde{l}_{1}(\xi)$, and, in particular,

$$
\left|\bar{h}_{2}(t, \xi)-\overline{\tilde{h}}_{2}(t, \xi)\right| \leqslant\left|h_{2}(t, \xi)-\tilde{h}_{2}(t, \xi)\right|+\left|l_{1}(\xi)-\tilde{l}_{1}(\xi)\right|,
$$

where $l_{1}(\xi)=\alpha\left(h_{2}\left(\tau_{2}(\xi), \xi\right)-l_{0}(\xi)\right)$ and $\tilde{l}_{1}(\xi)=\alpha\left(\tilde{h}_{2}\left(\tilde{\tau}_{2}(\xi), \xi\right)-l_{0}(\xi)\right)$. Thus we can write

$$
\begin{aligned}
& \left|l_{1}(\xi)-\tilde{l}_{1}(\xi)\right|=\alpha\left|h_{2}\left(\tau_{2}(\xi), \xi\right)-\tilde{h}_{2}\left(\tilde{\tau}_{2}(\xi), \xi\right)\right| \\
& \quad \leqslant \alpha\left(\left|h_{2}\left(\tau_{2}(\xi), \xi\right)-h_{2}\left(\tilde{\tau}_{2}(\xi), \xi\right)\right|+\left|h_{2}\left(\tilde{\tau}_{2}(\xi), \xi\right)-\tilde{h}_{2}\left(\tilde{\tau}_{2}(\xi), \xi\right)\right|\right) .
\end{aligned}
$$

The first term on the right-hand side can be estimated, using (54), as follows

$$
\begin{aligned}
\left|h_{2}\left(\tau_{2}(\xi), \xi\right)-h_{2}\left(\tilde{\tau}_{2}(\xi), \xi\right)\right| & \leqslant \int_{\tau_{2}}^{\tilde{\tau}_{2}} 2\left|U^{2}-P(\Theta)\right| w_{2}(t, \xi) d t \\
& \leqslant C(\bar{M}) \int_{\tau_{2}}^{\tilde{\tau}_{2}} w_{2}(t, \xi)-\tilde{w}_{2}(t, \xi) d t \\
& \leqslant C(\bar{M}) T\left\|X_{2}-\tilde{X}_{2}\right\|_{L_{T}^{\infty} \bar{v}},
\end{aligned}
$$

where we used that $w_{2}(t, \xi) \geqslant 0$ and $\tilde{w}_{2}(t, \xi) \leqslant 0$ for all $t \in\left[\tau_{2}(\xi), \tilde{\tau}_{2}(\xi)\right]$. Combining the above estimates yields

$$
\begin{aligned}
& \int_{\tilde{\tau}_{2}}^{T}\left|\bar{h}_{2}(t, \xi)-\overline{\tilde{h}}_{2}(t, \xi)\right| d t \leqslant \int_{\tilde{\tau}_{2}}^{T}\left|h_{2}(t, \xi)-\tilde{h}_{2}(t, \xi)\right| d t+T\left|l_{1}(\xi)-\tilde{l}_{1}(\xi)\right| \\
& \quad \leqslant \int_{\tilde{\tau}_{2}}^{T}\left|h_{2}(t, \xi)-\tilde{h}_{2}(t, \xi)\right| d t+\alpha T\left(\left|h_{2}\left(\tau_{2}(\xi), \xi\right)-h_{2}\left(\tilde{\tau}_{2}(\xi), \xi\right)\right|\right. \\
& \left.\quad+\left|h_{2}\left(\tilde{\tau}_{2}(\xi), \xi\right)-\tilde{h}_{2}\left(\tilde{\tau}_{2}(\xi), \xi\right)\right|\right) \\
& \quad \leqslant \int_{\tilde{\tau}_{2}}^{T}\left|h_{2}(t, \xi)-\tilde{h}_{2}(t, \xi)\right| d t+C(\bar{M}) T\left\|X_{2}-\tilde{X}_{2}\right\|_{L_{T}^{\infty} \bar{v}}
\end{aligned}
$$


Adding (93), (98), and (102), we obtain

$$
\begin{aligned}
& \int_{0}^{T}\left|\bar{h}_{2}(t, \xi)-\overline{\tilde{h}}_{2}(t, \xi)\right| d t \\
& \quad \leqslant 2 \int_{0}^{T}\left|h_{2}(t, \xi)-\tilde{h}_{2}(t, \xi)\right| d t+C(\bar{M})\left\|X_{2}-\tilde{X}_{2}\right\|_{L_{T}^{\infty} \bar{V}}
\end{aligned}
$$

Note that this inequality is true for all $\xi \in \kappa_{1-\gamma}$. Since meas $\left(\kappa_{1-\gamma}\right) \leqslant C(\bar{M})$, we can apply Fubini's theorem and use (103) to obtain

$$
\int_{0}^{T} \int_{\kappa_{1-\gamma}}\left|\bar{h}_{2}(t, \xi)-\overline{\tilde{h}}_{2}(t, \xi)\right| d \xi d t \leqslant C(\bar{M})\left\|X_{2}-\tilde{X}_{2}\right\|_{L_{T}^{\infty} \bar{v}}
$$

Combining (92) and (104) finally yields (87).

(iii) A close inspection of the proof of (ii) reveals that we only need to improve (98). Let us consider $\xi \in \kappa_{1-\gamma}$, and assume for the moment that $0<\tau_{2}(\xi)<$ $\tilde{\tau}_{2}(\xi) \leqslant T$, since all other cases can be derived from this one. For $t \in\left[\tau_{2}(\xi)\right.$, $\tilde{\tau}_{2}(\xi)$ ), we have

$$
\begin{aligned}
& \int_{\tau_{2}}^{\tilde{\tau}_{2}}\left|\bar{h}_{2}(t, \xi)-\overline{\tilde{h}}_{2}(t, \xi)\right| d t \\
& \quad \leqslant 2\left(w_{2}\left(\tau_{2}(\xi), \xi\right)-\tilde{w}_{2}\left(\tau_{2}(\xi), \xi\right)\right)+C(\bar{M}) T\left\|X_{2}-\tilde{X}_{2}\right\|_{L_{T}^{\infty} \bar{V}}
\end{aligned}
$$

In order to improve this estimate, we will use that $\Theta$ not only is an element of $C([0, T], \bar{V})$ like in (ii), but also belongs to $\operatorname{Im}(\mathcal{P})$. From $(54)$, we get that

$$
\begin{aligned}
& w_{2}\left(\tau_{2}(\xi), \xi\right)-\tilde{w}_{2}\left(\tau_{2}(\xi), \xi\right) \\
& \leqslant \frac{1}{2} \int_{0}^{\tau_{2}}\left|\bar{h}_{2}(t, \xi)-\overline{\tilde{h}}_{2}(t, \xi)\right| d t \\
&+\int_{0}^{\tau_{2}}\left|U^{2}-P(\Theta)\right|(t, \xi)\left|q_{2}(t, \xi)-\tilde{q}_{2}(t, \xi)\right| d t \\
&+\int_{0}^{\tau_{2}}\left|U^{2}-P(\Theta)-\tilde{U}^{2}+P(\tilde{\Theta})\right|(t, \xi) \tilde{q}_{2}(t, \xi) d t \\
& \leqslant \frac{1}{2} \int_{0}^{\tau_{2}}\left|h_{2}(t, \xi)-\tilde{h}_{2}(t, \xi)\right| d t+C(\bar{M}) T\left\|X_{2}-\tilde{X}_{2}\right\|_{L_{T}^{\infty} \bar{v}} \\
&+C(\bar{M}) \gamma\left\|U^{2}-P(\Theta)-\tilde{U}^{2}+P(\tilde{\Theta})\right\|_{L_{T}^{1} E} \\
& \leqslant C(\bar{M})\left(T\left(\left\|X_{2}-\tilde{X}_{2}\right\|_{L_{T}^{\infty} \bar{v}}+\|X-\tilde{X}\|_{L_{T}^{\infty} \bar{V}}\right)+\gamma \Gamma(\Theta, \tilde{\Theta})\right),
\end{aligned}
$$


where we used (85) and that $\left(q_{2} /\left(q_{2}+\bar{h}_{2}\right)\right)(t, \xi) \leqslant \gamma$ for all $t \in\left[0, \tau_{2}(\xi)\right]$, and therefore $q_{2}(t, \xi)=\left(q_{2}+\bar{h}_{2}\right)(t, \xi)\left(q_{2} /\left(q_{2}+\bar{h}_{2}\right)\right)(t, \xi) \leqslant C(\bar{M}) \gamma$ for all $t \in[0$, $\left.\tau_{2}(\xi)\right]$. Thus

$$
\begin{aligned}
& \int_{\tau_{2}}^{\tilde{\tau}_{2}}\left|\bar{h}_{2}(t, \xi)-\overline{\tilde{h}}_{2}(t, \xi)\right| d t \\
& \quad \leqslant 2\left(w_{2}\left(\tau_{2}(\xi), \xi\right)-\tilde{w}_{2}\left(\tau_{2}(\xi), \xi\right)\right)+C(\bar{M}) T\left\|X_{2}-\tilde{X}_{2}\right\|_{L_{T}^{\infty} \bar{v}} \\
& \quad \leqslant C(\bar{M})\left(T\left(\left\|X_{2}-\tilde{X}_{2}\right\|_{L_{T}^{\infty} \bar{v}}+\|X-\tilde{X}\|_{L_{T}^{\infty} \bar{v}}\right)+\gamma \Gamma(\Theta, \tilde{\Theta})\right) .
\end{aligned}
$$

As in (ii), we can conclude that, for all $\xi \in \kappa_{1-\gamma}$,

$$
\begin{aligned}
& \int_{0}^{T}\left|\bar{h}_{2}(t, \xi)-\overline{\tilde{h}}_{2}(t, \xi)\right| d t \\
& \quad \leqslant C(\bar{M})\left(T\left(\left\|X_{2}-\tilde{X}_{2}\right\|_{L_{T}^{\infty} \bar{V}}+\|X-\tilde{X}\|_{L_{T}^{\infty} \bar{V}}\right)+\gamma \Gamma(\Theta, \tilde{\Theta})\right) .
\end{aligned}
$$

Since meas $\left(\kappa_{1-\gamma}\right) \leqslant C(\bar{M})$, we can apply Fubini's theorem and use (106) to obtain

$$
\begin{aligned}
\int_{0}^{T} \int_{\kappa_{1-\gamma}} & \left|\bar{h}_{2}(t, \xi)-\overline{\tilde{h}}_{2}(t, \xi)\right| d \xi d t \\
& \leqslant C(\bar{M})\left(T\left(\left\|X_{2}-\tilde{X}_{2}\right\|_{L_{T}^{\infty} \bar{V}}+\|X-\tilde{X}\|_{L_{T}^{\infty} \bar{V}}\right)+\gamma \Gamma(\Theta, \tilde{\Theta})\right) .
\end{aligned}
$$

(iv) First, we estimate $\left\|Z_{2}-\tilde{Z}_{2}\right\|_{L_{T}^{\infty} \bar{W}\left(\kappa_{1-\gamma}^{c}\right)}$. For $\xi \in \kappa_{1-\gamma}^{c}$, we have $Z_{2}-\tilde{Z}_{2}=$ $\bar{Z}_{2}-\overline{\tilde{Z}}_{2}$, and, in particular, $\bar{Z}_{2, t}=F(\Theta) \bar{Z}_{2}$ and $\overline{\tilde{Z}}_{2, t}=F(\tilde{\Theta}) \overline{\tilde{Z}}_{2}$ for all $t \in[0, T]$. Hence,

$$
\begin{gathered}
\left\|\left(Z_{2}-\tilde{Z}_{2}\right)(t, \cdot)\right\|_{\bar{W}\left(\kappa_{1-\gamma}^{c}\right)}=\left\|\left(\bar{Z}_{2}-\overline{\tilde{Z}}_{2}\right)(t, \cdot)\right\|_{\bar{W}\left(\kappa_{1-\gamma}^{c}\right)} \\
\leqslant \int_{0}^{t}\left\|(F(\Theta)-F(\tilde{\Theta})) \bar{Z}_{2}\left(t^{\prime}, \cdot\right)\right\|_{\bar{W}\left(\kappa_{1-\gamma}^{c}\right)} d t^{\prime} \\
\quad+\int_{0}^{t}\left\|F(\tilde{\Theta})\left(\bar{Z}_{2}-\overline{\tilde{Z}}_{2}\right)\left(t^{\prime}, \cdot\right)\right\|_{\bar{W}\left(\kappa_{1-\gamma}^{c}\right)} d t^{\prime}
\end{gathered}
$$

We have that

$$
\begin{aligned}
(F(\Theta)-F(\tilde{\Theta})) \bar{Z}_{2}= & \left(0,\left(\left(U^{2}-P(\Theta)\right)-\left(\tilde{U}^{2}-P(\tilde{\Theta})\right)\right) q_{2},\right. \\
& \left.2\left(\left(U^{2}-P(\Theta)\right)-\left(\tilde{U}^{2}-P(\tilde{\Theta})\right)\right) w_{2}, 0\right),
\end{aligned}
$$

and therefore

$$
\left\|(F(\Theta)-F(\tilde{\Theta})) \bar{Z}_{2}\right\|_{L_{T}^{1} \bar{W}} \leqslant C(\bar{M})\left\|\left(U^{2}-P(\Theta)\right)-\left(\tilde{U}^{2}-P(\tilde{\Theta})\right)\right\|_{L_{T}^{1} E} .
$$


Applying Gronwall's lemma to (108), as $\|F(\tilde{\Theta})\|_{L_{T}^{\infty} L^{\infty}} \leqslant C(\bar{M})$, we get

$$
\left\|Z_{2}-\tilde{Z}_{2}\right\|_{L_{T}^{\infty} \bar{W}\left(\kappa_{1-\gamma}^{c}\right)} \leqslant C(\bar{M})\left\|(F(\Theta)-F(\tilde{\Theta})) \bar{Z}_{2}\right\|_{L_{T}^{1} \bar{W}} .
$$

Hence, we get by (110) that

$$
\left\|Z_{2}-\tilde{Z}_{2}\right\|_{L_{T}^{\infty} \bar{W}\left(\kappa_{1-\gamma}^{c}\right)} \leqslant C(\bar{M})\left\|\left(P(\Theta)-U^{2}\right)-\left(P(\tilde{\Theta})-\tilde{U}^{2}\right)\right\|_{L_{T}^{1} E} .
$$

Thus, we have by (85) that

$$
\left\|Z_{2}-\tilde{Z}_{2}\right\|_{L_{T}^{\infty} \bar{W}\left(\kappa_{1-\gamma}^{c}\right)} \leqslant C(\bar{M})\left(T\|X-\tilde{X}\|_{L_{T}^{\infty} \bar{V}}+\Gamma(\Theta, \tilde{\Theta})\right) .
$$

To estimate $\left\|Z_{2}-\tilde{Z}_{2}\right\|_{L_{T}^{\infty} \bar{W}\left(\kappa_{1-\gamma}\right)}$, we fix $\xi \in \kappa_{1-\gamma}$, and assume without loss of generality that $0<\tau_{2}(\xi)<\tilde{\tau}_{2}(\xi) \leqslant T$. For $t \in\left[0, \tau_{2}(\xi)\right]$, we can conclude as for $\xi \in \kappa_{1-\gamma}^{c}$ to obtain

$$
\left|Z_{2}(t, \xi)-\tilde{Z}_{2}(t, \xi)\right| \leqslant C(\bar{M})\left(T\|X-\tilde{X}\|_{L_{T}^{\infty} \bar{V}}+\Gamma(\Theta, \tilde{\Theta})\right) .
$$

For $t \in\left[\tau_{2}(\xi), \tilde{\tau}_{2}(\xi)\right)$, we have $\bar{Z}_{2, t}=F(\Theta) \bar{Z}_{2}$ and $\overline{\tilde{Z}}_{2, t}=F(\tilde{\Theta}) \overline{\tilde{Z}}_{2}$, but $h_{2}(t$, $\xi)-\tilde{h}_{2}(t, \xi)=\bar{h}_{2}(t, \xi)-\overline{\tilde{h}}_{2}(t, \xi)+l_{1}(\xi)$. Thus it follows, using (95), that

$$
\begin{aligned}
& \left|\left(Z_{2}-\tilde{Z}_{2}\right)(t, \xi)\right| \leqslant\left|\left(Z_{2}-\tilde{Z}_{2}\right)\left(\tau_{2}(\xi), \xi\right)\right|+\int_{\tau_{2}}^{t} \frac{1}{2} l_{1}(\xi) d t^{\prime} \\
& \quad+\int_{\tau_{2}}^{t}\left|(F(\Theta)-F(\tilde{\Theta})) Z_{2}\left(t^{\prime}, \xi\right)\right| d t^{\prime}+\int_{\tau_{2}}^{t}\left|F(\tilde{\Theta})\left(Z_{2}-\tilde{Z}_{2}\right)\left(t^{\prime}, \xi\right)\right| d t^{\prime} \\
& \leqslant\left|\left(Z_{2}-\tilde{Z}_{2}\right)\left(\tau_{2}(\xi), \xi\right)\right|+\int_{\tau_{2}}^{t} \frac{1}{2} \overline{\tilde{h}}_{2}\left(t^{\prime}, \xi\right) d t^{\prime} \\
& \quad+\int_{\tau_{2}}^{t}\left|(F(\Theta)-F(\tilde{\Theta})) Z_{2}\left(t^{\prime}, \xi\right)\right| d t^{\prime} \\
& \quad+\int_{\tau_{2}}^{t}(|F(\tilde{\Theta})|+1)\left|\left(Z_{2}-\tilde{Z}_{2}\right)\left(t^{\prime}, \xi\right)\right| d t^{\prime}
\end{aligned}
$$

where $(F(\Theta)-F(\tilde{\Theta})) Z_{2}=(F(\Theta)-F(\tilde{\Theta})) \bar{Z}_{2}$ is given by (109), which depends neither on $h_{2}$ and $\tilde{h}_{2}$ nor on $\bar{h}_{2}$ and $\tilde{\tilde{h}}_{2}$. Applying Gronwall's inequality then yields

$$
\begin{aligned}
\left|\left(Z_{2}-\tilde{Z}_{2}\right)(t, \xi)\right| \leqslant & C(\bar{M})\left(\left|\left(Z_{2}-\tilde{Z}_{2}\right)\left(\tau_{2}(\xi), \xi\right)\right|+\int_{\tau_{2}}^{t} \frac{1}{2} \overline{\tilde{h}}_{2}\left(t^{\prime}, \xi\right) d t^{\prime}\right. \\
& \left.+\int_{\tau_{2}}^{t}\left|(F(\Theta)-F(\tilde{\Theta})) Z_{2}\left(t^{\prime}, \xi\right)\right| d t^{\prime}\right)
\end{aligned}
$$


Then we get, from (114) and (110), together with

$$
\begin{aligned}
\int_{\tau_{2}}^{t} \frac{1}{2} \overline{\tilde{h}}_{2}\left(t^{\prime}, \xi\right) d t^{\prime} \leqslant & w_{2}\left(\tau_{2}(\xi), \xi\right)-\tilde{w}_{2}\left(\tau_{2}(\xi), \xi\right) \\
& +C(\bar{M}) T\left\|X_{2}(\cdot, \xi)-\tilde{X}_{2}(\cdot, \xi)\right\|_{L_{\tau_{2}}^{\infty}} \\
\leqslant & C(\bar{M})\left(T \left(\left\|X_{2}(\cdot, \xi)-\tilde{X}_{2}(\cdot, \xi)\right\|_{L_{\tau_{2}}^{\infty}}\right.\right. \\
& \left.\left.+\|X-\tilde{X}\|_{L_{T}^{\infty} \bar{V}}\right)+\gamma \Gamma(\Theta, \tilde{\Theta})\right) \\
\leqslant & C(\bar{M})\left(T\|X-\tilde{X}\|_{L_{T}^{\infty} \bar{V}}+(T+\gamma) \Gamma(\Theta, \tilde{\Theta})\right)
\end{aligned}
$$

where we used (97), (105), and (114), that

$$
\left|\left(Z_{2}-\tilde{Z}_{2}\right)(t, \xi)\right| \leqslant C(\bar{M})\left(T\|X-\tilde{X}\|_{L_{T}^{\infty} \bar{V}}+\Gamma(\Theta, \tilde{\Theta})\right) .
$$

For $t \in\left[\tilde{\tau}_{2}(\xi), T\right]$, we have $\bar{Z}_{2, t}=F(\Theta) \bar{Z}_{2}$ and $\overline{\tilde{Z}}_{2, t}=F(\tilde{\Theta}) \overline{\tilde{Z}}_{2}$, but $h_{2}(t$, $\xi)-\tilde{h}_{2}(t, \xi)=\bar{h}_{2}(t, \xi)-\overline{\tilde{h}}_{2}(t, \xi)+l_{1}(\xi)-\tilde{l}_{1}(\xi)$. Thus it follows that

$$
\begin{aligned}
\left|\left(Z_{2}-\tilde{Z}_{2}\right)(t, \xi)\right| \leqslant & \left|\left(Z_{2}-\tilde{Z}_{2}\right)\left(\tilde{\tau}_{2}(\xi), \xi\right)\right|+\int_{\tilde{\tau}_{2}}^{t} \frac{1}{2}\left|l_{1}(\xi)-\tilde{l}_{1}(\xi)\right| d t^{\prime} \\
& +\int_{\tilde{\tau}_{2}}^{t}\left|(F(\Theta)-F(\tilde{\Theta})) Z_{2}\left(t^{\prime}, \xi\right)\right| d t^{\prime} \\
& +\int_{\tilde{\tau}_{2}}^{t}\left|F(\tilde{\Theta})\left(Z_{2}-\tilde{Z}_{2}\right)\left(t^{\prime}, \xi\right)\right| d t^{\prime}
\end{aligned}
$$

where $(F(\Theta)-F(\tilde{\Theta})) Z_{2}=(F(\Theta)-F(\tilde{\Theta})) \bar{Z}_{2}$ is given by (109). Applying Gronwall's inequality then yields

$$
\begin{aligned}
\left|\left(Z_{2}-\tilde{Z}_{2}\right)(t, \xi)\right| \leqslant & C(\bar{M})\left(\left|\left(Z_{2}-\tilde{Z}_{2}\right)\left(\tilde{\tau}_{2}(\xi), \xi\right)\right|+\int_{\tilde{\tau}_{2}}^{t} \frac{1}{2}\left|l_{1}(\xi)-\tilde{l}_{1}(\xi)\right| d t^{\prime}\right. \\
& \left.+\int_{\tilde{\tau}_{2}}^{t}\left|(F(\Theta)-F(\tilde{\Theta})) Z_{2}\left(t^{\prime}, \xi\right)\right| d t^{\prime}\right)
\end{aligned}
$$

Then we get, from (118) and (110), together with

$$
\begin{aligned}
\left|l_{1}(\xi)-\tilde{l}_{1}(\xi)\right| \leqslant & \alpha\left|h_{2}\left(\tilde{\tau}_{2}(\xi), \xi\right)-\tilde{h}_{2}\left(\tilde{\tau}_{2}(\xi), \xi\right)\right| \\
& +C(\bar{M}) T\left\|X_{2}(\cdot, \xi)-\tilde{X}_{2}(\cdot, \xi)\right\|_{L_{\tilde{\tau}_{2}}^{\infty}} \\
\leqslant & C(\bar{M})\left(T\|X-\tilde{X}\|_{L_{T}^{\infty} \bar{V}}+\Gamma(\Theta, \tilde{\Theta})\right)
\end{aligned}
$$


where we used (100), (101), and (118), that

$$
\left|\left(Z_{2}-\tilde{Z}_{2}\right)(t, \xi)\right| \leqslant C(\bar{M})\left(T\|X-\tilde{X}\|_{L_{T}^{\infty} \bar{V}}+\Gamma(\Theta, \tilde{\Theta})\right) .
$$

Combining (114), (118), and (122), we get

$$
\left|\left(Z_{2}-\tilde{Z}_{2}\right)(t, \xi)\right| \leqslant C(\bar{M})\left(T\|X-\tilde{X}\|_{L_{T}^{\infty} \bar{V}}+\Gamma(\Theta, \tilde{\Theta})\right),
$$

for all $t \in[0, T]$. Since meas $\left(\kappa_{1-\gamma}\right) \leqslant C(\bar{M})$, the estimate (123) implies that

$$
\left\|Z_{2}-\tilde{Z}_{2}\right\|_{L_{T}^{\infty} \bar{W}\left(\kappa_{1-\gamma}\right)} \leqslant C(\bar{M})\left(T\|X-\tilde{X}\|_{L_{T}^{\infty} \bar{V}}+\Gamma(\Theta, \tilde{\Theta})\right) .
$$

Combining (113) and (124), we get

$$
\left\|Z_{2}-\tilde{Z}_{2}\right\|_{L_{T}^{\infty} \bar{W}} \leqslant C(\bar{M})\left(T\|X-\tilde{X}\|_{L_{T}^{\infty} \bar{V}}+\Gamma(\Theta, \tilde{\Theta})\right) .
$$

From (54), we obtain

$$
\begin{aligned}
\left\|U_{2}-\tilde{U}_{2}\right\|_{L_{T}^{\infty} E} & \leqslant\|Q(\Theta)-Q(\tilde{\Theta})\|_{L_{T}^{1} E} \\
& \leqslant C(\bar{M})\left(T\|X-\tilde{X}\|_{L_{T}^{\infty} \bar{V}}+\Gamma(\Theta, \tilde{\Theta})\right)
\end{aligned}
$$

and

$$
\begin{aligned}
\left\|\zeta_{2}-\tilde{\zeta}_{2}\right\|_{L_{T}^{\infty} L^{\infty}} & \leqslant T\left\|U_{2}-\tilde{U}_{2}\right\|_{L_{T}^{\infty} L^{\infty}} \\
& \leqslant C(\bar{M})\left(T\|X-\tilde{X}\|_{L_{T}^{\infty} \bar{V}}+\Gamma(\Theta, \tilde{\Theta})\right) .
\end{aligned}
$$

Thus, adding (125), (126), and (127), we conclude that

$$
\left\|X_{2}-\tilde{X}_{2}\right\|_{L_{T}^{\infty} \bar{V}} \leqslant C(\bar{M})\left(T\|X-\tilde{X}\|_{L_{T}^{\infty} \bar{V}}+\Gamma(\Theta, \tilde{\Theta})\right) .
$$

REMARK 14. Recall that, in the case of conservative solutions, that is, $\alpha=0$, we have that $\bar{h}(t, \xi)=h(t, \xi)$ for all $\xi \in \mathbb{R}$ and $t \in \mathbb{R}$, and hence the above proof simplifies considerably in that case. In particular, it suffices to prove (iv), since one can conclude that $\Gamma(\Theta, \tilde{\Theta}) \leqslant C(\bar{M}) T\|X-\tilde{X}\|_{L_{T}^{\infty} \bar{V}}$ as in (91).

THEOREM 15 (Short-time solution). Given $M>0$, for any initial data $\Theta_{0}=\left(y_{0}\right.$, $\left.U_{0}, y_{0, \xi}, U_{0, \xi}, \bar{h}_{0}, h_{0}, r_{0}\right) \in \mathcal{G} \cap B_{M}$, there exists a time $T>0$, which depends only on $M$, such that there exists a unique solution $\Theta=\left(y, U, y_{\xi}, U_{\xi}, \bar{h}, h, r\right) \in C([0$, $T], \bar{V})$ of $(32)$ with $\Theta(0)=\Theta_{0}$. Moreover, $\Theta(t) \in \mathcal{G}$ for all $t \in[0, T]$. 
Proof. In order to prove the existence and uniqueness of the solution, we use an iteration argument. By Lemma 11 , there exist $T$ and $\bar{M}$ such that $\mathcal{P}$ is a mapping from $C\left([0, T], B_{\bar{M}}\right)$ to $C\left([0, T], B_{\bar{M}}\right)$. Now, let $\Theta_{1}(t, \xi)=\Theta_{0}(\xi)$ for all $t \in[0$, $T$ ], and set $\Theta_{n+1}=\mathcal{P}\left(\Theta_{n}\right)$ and $\Theta_{n}(0, \xi)=\Theta_{0}(\xi)$ for all $n \in \mathbb{N}$. Then $\Theta_{n}$ belongs to $\operatorname{Im}(\mathcal{P})$ for all $n \in \mathbb{N}$, and, in particular, $\Theta_{n}(t) \in B_{\bar{M}}$ for all $t \in[0, T]$. We have

$$
\begin{aligned}
\left\|X_{n+1}-X_{n}\right\|_{L_{T}^{\infty} \bar{V} \leqslant} \leqslant & C(\bar{M})\left(T\left\|X_{n}-X_{n-1}\right\|_{L_{T}^{\infty} \bar{V}}+\Gamma\left(\Theta_{n}, \Theta_{n-1}\right)\right) \\
\leqslant & C(\bar{M})\left(T\left(\left\|X_{n}-X_{n-1}\right\|_{L_{T}^{\infty} \bar{V}}+\left\|X_{n-1}-X_{n-2}\right\|_{L_{T}^{\infty} \bar{V}}\right)\right. \\
& \left.+\gamma \Gamma\left(\Theta_{n-1}, \Theta_{n-2}\right)\right) \\
\leqslant & C(\bar{M})(T+\gamma)\left(\left\|X_{n}-X_{n-1}\right\|_{L_{T}^{\infty} \bar{V}}+\left\|X_{n-1}-X_{n-2}\right\|_{L_{T}^{\infty} \bar{V}}\right),
\end{aligned}
$$

where we used Lemma 13. Hence, for $T$ and $\gamma$ small enough, we have

$$
\left\|X_{n+1}-X_{n}\right\|_{L_{T}^{\infty} \bar{V}} \leqslant \frac{1}{4}\left(\left\|X_{n}-X_{n-1}\right\|_{L_{T}^{\infty} \bar{V}}+\left\|X_{n-1}-X_{n-2}\right\|_{L_{T}^{\infty} \bar{V}}\right) \quad \text { for } n \geqslant 3 .
$$

Summation over all $n \geqslant 3$ on the left-hand side then yields

$$
\sum_{n=3}^{N}\left\|X_{n+1}-X_{n}\right\|_{L_{T}^{\infty} \bar{V}} \leqslant \frac{1}{4}\left(\sum_{n=2}^{N-1}\left\|X_{n+1}-X_{n}\right\|_{L_{T}^{\infty} \bar{V}}+\sum_{n=1}^{N-2}\left\|X_{n+1}-X_{n}\right\|_{L_{T}^{\infty} \bar{V}}\right)
$$

and

$$
\frac{1}{2} \sum_{n=1}^{N}\left\|X_{n+1}-X_{n}\right\|_{L_{T}^{\infty} \bar{V}} \leqslant\left\|X_{2}-X_{1}\right\|_{L_{T}^{\infty} \bar{V}}+\left\|X_{3}-X_{2}\right\|_{L_{T}^{\infty} \bar{V}}
$$

independently of $N$. Thus, $\left\{X_{n}\right\}_{n=1}^{\infty}$ is a Cauchy sequence which converges to a unique limit $X$. In addition, Lemma 13(i)-(ii) implies that $h_{n}(t)$ converges to a unique limit $h(t)$ in $L^{1}(\mathbb{R}) \cap L^{2}(\mathbb{R})$, and that $\bar{h}_{n}(t)$ converges to a unique limit $\bar{h}(t)$ in $L^{1}(\mathbb{R}) \cap L^{2}(\mathbb{R})$.

Next, we want to show that, for almost every $\xi \in \mathbb{R}$ such that $\tau_{1}(\xi) \leqslant T$, we have

$$
X\left(\tau_{1}(\xi), \xi\right)=X\left(\tau_{1}(\xi)-0, \xi\right) \quad \text { and } \quad \bar{h}\left(\tau_{1}(\xi), \xi\right)=(1-\alpha) \bar{h}\left(\tau_{1}(\xi)-0, \xi\right)
$$

Let $\mathcal{A}$ be the following set:

$$
\begin{aligned}
\mathcal{A}= & \left\{\xi \in \mathbb{R}|| \zeta_{0}(\xi)\left|\leqslant\left\|\zeta_{0}\right\|_{L^{\infty}},\right| U_{0}(\xi) \mid \leqslant\left\|U_{0}\right\|_{L^{\infty}},\right. \\
& \left|q_{0}(\xi)-1\right| \leqslant\left\|q_{0}-1\right\|_{L^{\infty}},\left|w_{0}(\xi)\right| \leqslant\left\|w_{0}\right\|_{L^{\infty}}, \\
& \left.\left|h_{0}(\xi)\right| \leqslant\left\|h_{0}\right\|_{L^{\infty}},\left|r_{0}(\xi)\right| \leqslant\left\|r_{0}\right\|_{L^{\infty}}\right\} .
\end{aligned}
$$


We have that $\mathcal{A}$ has full measure; that is, meas $\left(\mathcal{A}^{c}\right)=0$. Recall that

$$
\begin{aligned}
\|X(\cdot, \xi)\|_{L_{T}^{\infty}}= & \sup _{t \in[0, T]}\{|y(t, \xi)-\xi|+|U(t, \xi)|+|q(t, \xi)| \\
& +|w(t, \xi)|+|h(t, \xi)|+|r(t, \xi)|\} .
\end{aligned}
$$

Since both $P\left(\Theta_{n}\right)(t, \xi)$ and $Q\left(\Theta_{n}\right)(t, \xi)$ belong to $H^{1}(\mathbb{R})$ for all $t \in[0, T]$, we have that

$$
\sup _{t \in[0, T]}\left\{\left|P\left(\Theta_{n}\right)(t, \xi)\right|+\left|Q\left(\Theta_{n}\right)(t, \xi)\right|\right\} \leqslant\left\|P\left(\Theta_{n}\right)\right\|_{L_{T}^{\infty} E}+\left\|Q\left(\Theta_{n}\right)\right\|_{L_{T}^{\infty} E} \leqslant C(\bar{M}) .
$$

Moreover, following closely the proof of Lemma 11, we obtain that, for any $\xi \in$ $\mathcal{A}$,

$$
\left\|X_{n}(\cdot, \xi)\right\|_{L_{T}^{\infty}} \leqslant\left\|X_{n}\right\|_{L_{T}^{\infty} \bar{V}} \leqslant C(\bar{M}),
$$

which implies that $X_{n}(t, \xi)$ is continuous with respect to time. In particular, one obtains that, for any $\xi \in \mathcal{A}$,

$$
\left\|X_{n}(\cdot, \xi)-X_{n-1}(\cdot, \xi)\right\|_{L_{T}^{\infty}} \leqslant\left\|X_{n}-X_{n-1}\right\|_{L_{T}^{\infty} \bar{V}}
$$

Thus $\left\|X_{n}(\cdot, \xi)-X_{n-1}(\cdot, \xi)\right\|_{L_{T}^{\infty}} \rightarrow 0$, and $X_{n}(\xi)$ converges to the unique limit $X(\xi)$ for almost every $\xi \in \mathbb{R}$. Thus, if we can show that

$$
\bar{h}\left(\tau_{1}(\xi), \xi\right)=\bar{h}\left(\tau_{1}(\xi)+0, \xi\right)=(1-\alpha) \bar{h}\left(\tau_{1}(\xi)-0, \xi\right),
$$

for all $\xi \in \mathcal{A}$ that experience wave breaking within $[0, T], \Theta$ will be a solution of (32) in the sense of Definition 1. Recall that for any $n \in \mathbb{N}$ we have that

$$
\bar{h}_{n}\left(\tau_{1, n}(\xi), \xi\right)=\bar{h}_{n}\left(\tau_{1, n}(\xi)+0, \xi\right)=(1-\alpha) \bar{h}_{n}\left(\tau_{1, n}(\xi)-0, \xi\right) .
$$

Thus, if we can show that $\tau_{1, n}(\xi)$ converges to a unique limit $\tau_{1}(\xi)$, the claim will follow, since $\left\|X_{n}(\cdot, \xi)-X(\cdot, \xi)\right\|_{L_{T}^{\infty}} \rightarrow 0$. We assume without loss of generality that $0<\tau_{1, n-1}(\xi)<\tau_{1, n}(\xi) \leqslant T$, since all other possible cases can be handled similarly. Moreover, we assume that $q_{0}(\xi)+h_{0}(\xi)-l_{0}(\xi)=C>0$, since otherwise $\bar{h}_{0}(\xi)=0=q_{0}(\xi)$, and (131) is obviously satisfied. Then, as in the proof of Lemma 10(ii), we can find a strictly positive constant $C_{T}$ such that $C_{T}<q(t, \xi)+h(t, \xi)-l_{0}(\xi)$ for all $t \in[0, T]$. In particular, we get

$$
\begin{aligned}
\tau_{1, n}(\xi)-\tau_{1, n-1}(\xi)= & \int_{\tau_{1, n-1}(\xi)}^{\tau_{1, n}(\xi)} \frac{q_{n}(s, \xi)+\bar{h}_{n}(s, \xi)}{q_{n}(s, \xi)+\bar{h}_{n}(s, \xi)} d s \\
& \leqslant C_{T}^{-1} \int_{\tau_{1, n-1}(\xi)}^{\tau_{1, n}(\xi)}\left(q_{n}+\bar{h}_{n}\right)(s, \xi) d s
\end{aligned}
$$


where we used that $\bar{h}_{n}(t, \xi)=h_{n}(t, \xi)-l_{0}(\xi)$ for $t \in\left[0, \tau_{1, n}(\xi)\right)$. We split the integral on the right-hand side into two parts, and study them separately. For the first integral, we get

$$
\begin{aligned}
\int_{\tau_{1, n-1}(\xi)}^{\tau_{1, n}(\xi)} q_{n}(s, \xi) d s & =\int_{\tau_{1, n-1}(\xi)}^{\tau_{1, n}(\xi)}\left(q_{n}(s, \xi)-q_{n}\left(\tau_{1, n-1}(\xi), \xi\right)+q_{n}\left(\tau_{1, n-1}(\xi), \xi\right)\right) d s \\
& \leqslant \int_{\tau_{1, n-1}(\xi)}^{\tau_{1, n}(\xi)} q_{n}\left(\tau_{1, n-1}(\xi), \xi\right) d s \\
& =\int_{\tau_{1, n-1}(\xi)}^{\tau_{1, n}(\xi)}\left(q_{n}\left(\tau_{1, n-1}(\xi), \xi\right)-q_{n-1}\left(\tau_{1, n-1}(\xi), \xi\right)\right) d s \\
& \leqslant T\left\|q_{n}(\cdot, \xi)-q_{n-1}(\cdot, \xi)\right\|_{L_{T}^{\infty}} \\
& \leqslant T\left\|X_{n}-X_{n-1}\right\|_{L_{T}^{\infty} \bar{V}},
\end{aligned}
$$

where we used that $q_{n}(s, \xi)$ is decreasing on the interval $\left[\tau_{1, n-1}(\xi), \tau_{1, n}(\xi)\right]$, since $q_{n, t}(s, \xi)=w_{n}(s, \xi) \leqslant 0$ for all $s \in\left[\tau_{1, n-1}(\xi), \tau_{1, n}(\xi)\right]$, and that $q_{n-1}\left(\tau_{1, n-1}(\xi), \xi\right)=0$. As far as the second integral is concerned, we can conclude as follows:

$$
\begin{aligned}
\int_{\tau_{1, n-1}(\xi)}^{\tau_{1, n}(\xi)} \bar{h}_{n}(s, \xi) d s= & 2\left(w_{n}\left(\tau_{1, n}(\xi), \xi\right)-w_{n}\left(\tau_{1, n-1}(\xi), \xi\right)\right) \\
& -2 \int_{\tau_{1, n-1}(\xi)}^{\tau_{1, n}(\xi)}\left(U_{n-1}^{2}-P\left(\Theta_{n-1}\right)\right) q_{n}(s, \xi) d s \\
\leqslant & -2 w_{n}\left(\tau_{1, n-1}(\xi), \xi\right)+C(\bar{M}) T q_{n}\left(\tau_{1, n-1}(\xi), \xi\right) \\
\leqslant & 2\left(w_{n-1}\left(\tau_{1, n-1}(\xi), \xi\right)-w_{n}\left(\tau_{1, n-1}(\xi), \xi\right)\right) \\
& +C(\bar{M}) T\left(q_{n}\left(\tau_{1, n-1}(\xi), \xi\right)-q_{n-1}\left(\tau_{1, n-1}(\xi), \xi\right)\right) \\
\leqslant & \left\|w_{n}(\cdot, \xi)-w_{n-1}(\cdot, \xi)\right\|_{L_{T}^{\infty}} \\
& +C(\bar{M}) T\left\|q_{n}(\cdot, \xi)-q_{n-1}(\cdot, \xi)\right\|_{L_{T}^{\infty}} \\
\leqslant & (1+T C(\bar{M}))\left\|X_{n}-X_{n-1}\right\|_{L_{T}^{\infty} \bar{V}}
\end{aligned}
$$

where we used $w_{n}\left(\tau_{1, n}(\xi), \xi\right)=w_{n-1}\left(\tau_{1, n-1}(\xi), \xi\right)=q_{n-1}\left(\tau_{1, n-1}(\xi), \xi\right)=0$. Thus, the sequence $\tau_{1, n}(\xi)$ converges to a unique limit $\tau_{1}(\xi)$ for every $\xi \in \mathcal{A}$, and, in particular, $\lim _{n \rightarrow \infty} h_{n}\left(\tau_{1, n}(\xi), \xi\right)=h\left(\tau_{1}(\xi), \xi\right)$ for all $\xi \in \mathcal{A}$. This implies, since $\bar{h}(t, \xi)=\lim _{n \rightarrow \infty} \bar{h}_{n}(t, \xi)$ for $t \neq \tau_{1}(\xi)$, that

$$
\begin{aligned}
\bar{h}\left(\tau_{1}(\xi), \xi\right) & =\lim _{s \downarrow \tau_{1}(\xi)} \bar{h}(s, \xi) \\
& =\lim _{s \downarrow \tau_{1}(\xi)} \lim _{n \rightarrow \infty} \bar{h}_{n}(s, \xi) \\
& =\lim _{s \downarrow \tau_{1}(\xi)} \lim _{n \rightarrow \infty}\left(h_{n}(s, \xi)-l_{1, n}(\xi)-l_{0}(\xi)\right)
\end{aligned}
$$




$$
\begin{aligned}
& =\lim _{s \downarrow \tau_{1}(\xi)} \lim _{n \rightarrow \infty}\left(h_{n}(s, \xi)-\alpha\left(h_{n}\left(\tau_{1, n}(\xi), \xi\right)-l_{0}(\xi)\right)-l_{0}(\xi)\right) \\
& =\lim _{s \downarrow \tau_{1}(\xi)}\left(h(s, \xi)-\alpha\left(h\left(\tau_{1}(\xi), \xi\right)-l_{0}(\xi)\right)-l_{0}(\xi)\right) \\
& =h\left(\tau_{1}(\xi), \xi\right)-\alpha\left(h\left(\tau_{1}(\xi), \xi\right)-l_{0}(\xi)\right)-l_{0}(\xi) \\
& =(1-\alpha)\left(h\left(\tau_{1}(\xi), \xi\right)-l_{0}(\xi)\right)
\end{aligned}
$$

and

$$
\begin{aligned}
\bar{h}\left(\tau_{1}(\xi)-0, \xi\right) & =\lim _{s \uparrow \tau_{1}(\xi)} \bar{h}(s, \xi) \\
& =\lim _{s \uparrow \tau_{1}(\xi)} \lim _{n \rightarrow \infty} \bar{h}_{n}(s, \xi) \\
& =\lim _{s \uparrow \tau_{1}(\xi)} \lim _{n \rightarrow \infty}\left(h_{n}(s, \xi)-l_{0}(\xi)\right) \\
& =\lim _{s \uparrow \tau_{1}(\xi)} h(s, \xi)-l_{0}(\xi) \\
& =h\left(\tau_{1}(\xi), \xi\right)-l_{0}(\xi) .
\end{aligned}
$$

Thus,

$$
\bar{h}\left(\tau_{1}(\xi), \xi\right)=(1-\alpha)\left(h\left(\tau_{1}(\xi), \xi\right)-l_{0}(\xi)\right)=(1-\alpha) \bar{h}\left(\tau_{1}(\xi)-0, \xi\right),
$$

and, in particular,

$$
\bar{h}(t, \xi)= \begin{cases}h(t, \xi)-l_{0}(\xi) & \text { for } t<\tau_{1}(\xi) \\ h(t, \xi)-l_{0}(\xi)-l_{1}(\xi) & \text { otherwise }\end{cases}
$$

where $l_{1}(\xi)=\alpha\left(h\left(\tau_{1}(\xi), \xi\right)-l_{0}(\xi)\right)=\lim _{n \rightarrow \infty} l_{1, n}(\xi)$.

It is left to prove that $U$ and $y$ are differentiable, and that $U_{\xi}=w$ and $y_{\xi}=q$. Recall that $Q(\Theta)$ is defined via (40), and choose $\xi_{1}, \xi_{2} \in \mathbb{R}$ such that $\xi_{1}<\xi_{2}$. Then we have

$$
\begin{aligned}
& \int_{-\infty}^{\xi_{2}} e^{-\left|y\left(t, \xi_{2}\right)-y(t, \eta)\right|}\left(2 U^{2} q+\bar{h}\right)(t, \eta) d \eta \\
& \quad-\int_{-\infty}^{\xi_{1}} e^{-\left|y\left(t, \xi_{1}\right)-y(t, \eta)\right|}\left(2 U^{2} q+\bar{h}\right)(t, \eta) d \eta \\
& =\int_{-\infty}^{\xi_{1}}\left(e^{-\left|y\left(t, \xi_{2}\right)-y(t, \eta)\right|}-e^{-\left|y\left(t, \xi_{1}\right)-y(t, \eta)\right|}\right)\left(2 U^{2} q+\bar{h}\right)(t, \eta) d \eta \\
& \quad+\int_{\xi_{1}}^{\xi_{2}} e^{-\left|y\left(t, \xi_{2}\right)-y(t, \eta)\right|}\left(2 U^{2} q+\bar{h}\right)(t, \eta) d \eta \\
& \leqslant C(\bar{M})\left(\left|y\left(t, \xi_{2}\right)-y\left(t, \xi_{1}\right)\right|+\xi_{2}-\xi_{1}\right) .
\end{aligned}
$$


Here, we used that

$$
\begin{aligned}
\left|e^{-\left|y\left(t, \xi_{2}\right)-y(t, \eta)\right|}-e^{-\left|y\left(t, \xi_{1}\right)-y(t, \eta)\right|}\right| & =\left|\int_{-\mid y\left(t, \xi_{1}\right)-y(t, \eta)}^{-\left|y\left(t, \xi_{2}\right)-y(t, \eta)\right|} e^{x} d x\right| \\
& \leqslant|| y\left(t, \xi_{1}\right)-y(t, \eta)|-| y\left(t, \xi_{2}\right)-y(t, \eta) \| \\
& \leqslant\left|y\left(t, \xi_{1}\right)-y\left(t, \xi_{2}\right)\right| .
\end{aligned}
$$

Thus (140) implies that $Q(\Theta)$ is differentiable almost everywhere according to Rademacher's theorem if $y(t, \xi)$ is Lipschitz continuous, since

$$
Q(\Theta)\left(t, \xi_{2}\right)-Q(\Theta)\left(t, \xi_{1}\right) \leqslant C(\bar{M})\left(\left|y\left(t, \xi_{2}\right)-y\left(t, \xi_{1}\right)\right|+\xi_{2}-\xi_{1}\right) .
$$

Therefore, observe that

$$
y\left(t, \xi_{2}\right)-y\left(t, \xi_{1}\right)=\int_{0}^{t}\left(U\left(s, \xi_{2}\right)-U\left(s, \xi_{1}\right)\right) d s+y\left(0, \xi_{2}\right)-y\left(0, \xi_{1}\right)
$$

and

$U\left(t, \xi_{2}\right)-U\left(t, \xi_{1}\right)=-\int_{0}^{t}\left(Q(\Theta)\left(s, \xi_{2}\right)-Q(\Theta)\left(s, \xi_{1}\right)\right) d s+U\left(0, \xi_{2}\right)-U\left(0, \xi_{1}\right)$,

where $U(0, \xi)$ and $y(0, \xi)$ are Lipschitz continuous due to the assumptions on the initial data. Combining these two inequalities and (142) yields

$$
\begin{aligned}
\left|y\left(t, \xi_{2}\right)-y\left(t, \xi_{1}\right)\right| \leqslant & \int_{0}^{t}\left|U\left(s, \xi_{2}\right)-U\left(s, \xi_{1}\right)\right| d s+\left|y\left(0, \xi_{2}\right)-y\left(0, \xi_{1}\right)\right| \\
\leqslant & \int_{0}^{t} \int_{0}^{s}\left|Q(\Theta)\left(r, \xi_{2}\right)-Q(\Theta)\left(r, \xi_{1}\right)\right| d r d s \\
& +\int_{0}^{t}\left|U\left(0, \xi_{2}\right)-U\left(0, \xi_{1}\right)\right| d s+\left|y\left(0, \xi_{2}\right)-y\left(0, \xi_{1}\right)\right| \\
\leqslant & C(\bar{M})\left(\int_{0}^{t}\left|y\left(s, \xi_{2}\right)-y\left(s, \xi_{1}\right)\right| d s+\xi_{2}-\xi_{1}\right)
\end{aligned}
$$

Applying Gronwall's inequality yields

$$
\left|y\left(t, \xi_{2}\right)-y\left(t, \xi_{1}\right)\right| \leqslant C(\bar{M})\left|\xi_{2}-\xi_{1}\right| .
$$

Thus $y(t, \xi)$ is Lipschitz continuous and differentiable almost everywhere. As an immediate consequence, we get from (142) and (144) that also $Q$ and $U$ are Lipschitz continuous and therefore differentiable almost everywhere. 
We are now ready to show that $w=U_{\xi}$ and $q=y_{\xi}$. Therefore, recall that $Q(\Theta)$ is defined via (40), and note that $Q(\Theta)$ is differentiable since $y$ is differentiable. A direct computation gives us that

$$
Q_{\xi}(\Theta)=-\frac{1}{2} \bar{h}-U^{2} q+P(\Theta) y_{\xi} .
$$

In addition, as $q(t, \xi)$ and $w(t, \xi)$ are both continuous with respect to time, we have

$$
\begin{gathered}
\left(q-y_{\xi}\right)_{t}=\left(w-U_{\xi}\right), \\
\left(w-U_{\xi}\right)_{t}=-P(\Theta)\left(q-y_{\xi}\right) .
\end{gathered}
$$

In particular, this means that, if $q_{0}=y_{0, \xi}$ and $w_{0}=U_{0, \xi}$, then

$$
\begin{aligned}
& \left\|\left(q-y_{\xi}\right)(t, \cdot)\right\|_{E}+\left\|\left(w-U_{\xi}\right)(t, \cdot)\right\|_{E} \\
& \quad \leqslant C(\bar{M}) \int_{0}^{t}\left(\left\|\left(q-y_{\xi}\right)\left(t^{\prime}, \cdot\right)\right\|_{E}+\left\|\left(w-U_{\xi}\right)\left(t^{\prime}, \cdot\right)\right\|_{E}\right) d t^{\prime},
\end{aligned}
$$

and thus using Gronwall's inequality yields that $y_{\xi}=q$ and $U_{\xi}=w$.

Let us prove that $X(t) \in \mathcal{G}$ for all $t$. From (58) and (59), we get $q(t, \xi) \geqslant 0$, $h(t, \xi) \geqslant 0$, and $q \bar{h}=w^{2}+r^{2}$ for all $t$ and almost all $\xi$, and therefore, since $U_{\xi}=w$ and $y_{\xi}=q$, conditions (37d) and (37g) are fulfilled, and $y$ is an increasing function. Since $\zeta(t, \xi)=\zeta(0, \xi)+\int_{0}^{t} U(t, \xi) d t$, we obtain by the Lebesgue dominated convergence theorem that $\lim _{\xi \rightarrow-\infty} \zeta(t, \xi)=0$, because $U \in H^{1}(\mathbb{R})$. Hence, since in addition $X(t) \in B_{\bar{M}}$, the function $X(t)$ fulfills all the conditions listed in (37), and thus $X(t) \in \mathcal{G}$.

Note that the set $\mathcal{G} \cap B_{M}$ is closed with respect to the topology of $\bar{V}$. We have

$$
\begin{gathered}
y_{\xi, t}=U_{\xi}, \\
h_{t}=2\left(U^{2}-P(\Theta)\right) U_{\xi}, \\
r_{t}=0,
\end{gathered}
$$

for all $\xi \in \mathbb{R}$ and $t \in \mathbb{R}_{+}$. In particular, this means that $y_{\xi}, h$, and $r$ are differentiable with respect to time in the classical sense almost everywhere.

In order to obtain global solutions, we want to apply Theorem 15 iteratively, which is possible if we can show that $\|X\|_{\bar{V}}+\|h\|_{L^{1}}+\left\|1 /\left(y_{\xi}+h\right)\right\|_{L^{\infty}}$ does not blow up within finite time. The corresponding estimate is contained in the following lemma.

Lemma 16. Given $M$ and $T_{0}$, then there exists a constant $M_{0}$ which depends only on $M$ and $T_{0}$ such that, for any $\Theta_{0}=\left(y_{0}, U_{0}, y_{0, \xi}, U_{0, \xi}, \bar{h}_{0}, h_{0}, r_{0}\right) \in B_{M}$, the 
following hold for all $t \in\left[0, T_{0}\right]$ :

$$
\|X(t)\|_{\bar{V}}+\|h(t)\|_{L^{1}}+\left\|\frac{1}{y_{\xi}+h}(t)\right\|_{L^{\infty}} \leqslant M_{0}
$$

and

$$
\int_{\mathbb{R}}\left(U^{2} y_{\xi}(t, \xi)+h(t, \xi)\right) d \xi=\int_{\mathbb{R}}\left(U_{0}^{2} y_{0, \xi}(\xi)+h_{0}(\xi)\right) d \xi .
$$

Proof. This proof follows the same lines as the one in [27]. To simplify the notation, we will generically denote by $C$ constants, and by $C\left(M, T_{0}\right)$ constants which in addition depend on $M$ and $T_{0}$. Let us introduce

$$
\Sigma(t)=\int_{\mathbb{R}}\left(U^{2} y_{\xi}+h\right)(t, \xi) d \xi .
$$

Since $h \geqslant 0$, we have $\|h\|_{L_{\mathbb{R}}^{1}}=\int_{\mathbb{R}} h d \xi<\infty$. After some computation, (32) yields that

$$
\Sigma(t)=\Sigma(0) \quad \text { for all } t \in \mathbb{R}_{+},
$$

which implies that

$$
\|h(t, \cdot)\|_{L^{1}} \leqslant \Sigma(0)
$$

Moreover, we have

$$
\begin{aligned}
U^{2}(t, \xi) & =2 \int_{-\infty}^{\xi} U U_{\xi}(t, \eta) d \eta \\
& \leqslant \int_{\left\{\eta \mid y_{\xi}(\eta)>0\right\}}\left(U^{2} y_{\xi}+\frac{U_{\xi}^{2}}{y_{\xi}}\right)(t, \eta) d \eta \\
& \leqslant \int_{\left\{\eta \mid y_{\xi}(\eta)>0\right\}}\left(U^{2} y_{\xi}+h\right)(t, \eta) d \eta \\
& \leqslant \Sigma(t)=\Sigma(0),
\end{aligned}
$$

where we used that $y_{\xi}(\eta)=0$ implies that $U_{\xi}(\eta)=0$, and therefore the integrand in the integral in the first line vanishes whenever $y_{\xi}(\xi)=0$. Thus it suffices to integrate over $\left\{\eta \in \mathbb{R} \mid y_{\xi}(\eta)>0\right\} \cap\{\eta \leqslant \xi\}$, which justifies the subsequent estimate. Thus

$$
\|U(t, \cdot)\|_{L^{\infty}}^{2} \leqslant \Sigma(0)
$$

Moreover, $P$ and $Q$ satisfy

$$
\|P(\Theta)(t, \cdot)\|_{L^{\infty}} \leqslant 2 \Sigma(0) \quad \text { and } \quad\|Q(\Theta)(t, \cdot)\|_{L^{\infty}} \leqslant 2 \Sigma(0) .
$$


From (32), we obtain that

$$
|\zeta(t, \xi)| \leqslant|\zeta(0, \xi)|+\int_{0}^{t}\left|U\left(t^{\prime}, \xi\right)\right| d t^{\prime},
$$

and hence

$$
\|\zeta(t, \cdot)\|_{L^{\infty}} \leqslant\|\zeta(0, \cdot)\|_{L^{\infty}}+T(1+\Sigma(0))
$$

Applying Young's inequality to (33) and (34), and following the proof of Lemma 8, we get

$$
\|P(\Theta)(t, \cdot)\|_{L^{2}}+\|Q(\Theta)(t, \cdot)\|_{L^{2}} \leqslant C e^{2\|\zeta(t, \cdot)\|_{L^{\infty}}} \Sigma(0) .
$$

Let

$$
\alpha(t)=\|U(t, \cdot)\|_{E}+\left\|\zeta_{\xi}(t, \cdot)\right\|_{E}+\left\|U_{\xi}(t, \cdot)\right\|_{E}+\|h(t, \cdot)\|_{E}+\|r(t, \cdot)\|_{E} .
$$

Then

$$
\alpha(t) \leqslant \alpha(0)+C\left(M, T_{0}\right)+C\left(M, T_{0}\right) \int_{0}^{t} \alpha\left(t^{\prime}\right) d t^{\prime} .
$$

Hence, Gronwall's lemma gives us $\alpha(t) \leqslant C\left(M, T_{0}\right)$. It remains to prove that $\left\|1 /\left(y_{\xi}+h\right)\right\|_{L_{T}^{\infty} L^{\infty}}$ can be bounded by some constant depending on $M$ and $T_{0}$, but this follows immediately from (60). This completes the proof.

We can now prove global existence of solutions.

THEOREM 17 (Global solution). For any initial data $\Theta_{0}=\left(y_{0}, U_{0}, y_{0, \xi}, U_{0, \xi}\right.$, $\left.h_{0}, \bar{h}_{0}, r_{0}\right) \in \mathcal{G}$, there exists a unique global solution $\Theta=\left(y, U, y_{\xi}, U_{\xi}, \bar{h}, h\right.$, $r) \in C\left(\mathbb{R}_{+}, \mathcal{G}\right)$ of $(32)$ with $\Theta(0)=\Theta_{0}$.

Proof. By assumption, $\Theta_{0} \in \mathcal{G}$, and therefore there exists a constant $M$ such that $\Theta_{0} \in B_{M}$. By Theorem 15, there exists a $T>0$, dependent on $M$, such that we can find a unique solution $\Theta(t) \in \mathcal{G}$ on $[0, T]$. Thus, we can find a global solution to (32) if and only if $\|X(t)\|_{\bar{V}}+\|h(t)\|_{L^{1}}+\left\|1 /\left(y_{\xi}+h\right)(t)\right\|_{L^{\infty}}$ does not blow up within a finite time interval, but this follows from Lemma 16.

Observe that $\left(\zeta, U, \zeta_{\xi}, U_{\xi}, \bar{h}, h, r\right)$ is a fixed point of $\mathcal{P}$, and that the results of Lemma 10 hold for $\Theta=\tilde{\Theta}=\left(\zeta, U, \zeta_{\xi}, U_{\xi}, \bar{h}, h, r\right)$. Since this lemma contains important information about which points will experience wave breaking in the near future, we rewrite it for the fixed-point solution $\Theta$. For this purpose, we redefine $B_{M}$ and $\kappa_{1-\gamma}$, see (43) and (56), as

$$
B_{M}=\left\{\Theta \mid\|X\|_{\bar{V}}+\|h\|_{L^{1}}+\left\|\frac{1}{y_{\xi}+h}\right\|_{L^{\infty}} \leqslant M\right\},
$$


where $X=\left(\zeta, U, \zeta_{\xi}, U_{\xi}, h, r\right)$, and

$$
\begin{aligned}
\kappa_{1-\gamma}= & \left\{\xi \in \mathbb{R} \mid \frac{\bar{h}_{0}}{y_{0, \xi}+\bar{h}_{0}}(\xi) \geqslant 1-\gamma, U_{0, \xi}(\xi) \leqslant 0, \text { and } r_{0}(\xi)=0\right\}, \\
& \gamma \in\left[0, \frac{1}{2}\right] .
\end{aligned}
$$

Note that every condition imposed on points $\xi \in \kappa_{1-\gamma}$ is motivated by what is known about wave breaking. If wave breaking occurs at some time $t_{b}$, then energy is concentrated on sets of measure zero in Eulerian coordinates, which correspond to the sets where $\left(h /\left(y_{\xi}+h\right)\right)\left(t_{b}, \xi\right)=1=\left(\bar{h} /\left(y_{\xi}+\bar{h}\right)\right)\left(t_{b}-0, \xi\right)$ in Lagrangian coordinates. Furthermore, it is well known that wave breaking in the context of the $2 \mathrm{CH}$ system means that the spatial derivative becomes unbounded from below, and hence $U_{\xi}(t, \xi) \leqslant 0$ for $t_{b}-\delta \leqslant t \leqslant t_{b}$ for such points; see [11, 20]. Finally, it has been shown in [15, Theorem 6.1] that wave breaking within finite time can only occur at points $\xi$ where $r_{0}(\xi)=0$.

Lemma 10 can be rewritten, due to (154) and (155), as follows.

COROLlary 18. Let $M_{0}$ be a constant, and consider initial data $\Theta_{0} \in \mathcal{G} \cap B_{M}$. Denote by $\Theta=\left(\zeta, U, \zeta_{\xi}, U_{\xi}, \bar{h}, h, r\right) \in C\left(\mathbb{R}_{+}, \mathcal{G}\right)$ the global solution of (32) with initial data $\Theta_{0}$. Then the following statements hold.

(i) We have

$$
\left\|\frac{1}{y_{\xi}+h}(t, \cdot)\right\|_{L^{\infty}} \leqslant 2 e^{C(M) T}\left\|\frac{1}{y_{0, \xi}+h_{0}}\right\|_{L^{\infty}},
$$

and

$$
\left\|\left(y_{\xi}+h\right)(t, \cdot)\right\|_{L^{\infty}} \leqslant 2 e^{C(M) T}\left\|y_{0, \xi}+h_{0}\right\|_{L^{\infty}}
$$

for all $t \in[0, T]$ and a constant $C(M)$ which depends on $M$.

(ii) There exists a $\gamma \in\left(0, \frac{1}{2}\right)$ depending only on $M$ such that, if $\xi \in \kappa_{1-\gamma}$, then $\Theta(t, \xi) \in \Omega_{1}$ for all $t \in\left[0, \min \left(\tau_{1}(\xi), T\right)\right],\left(y_{\xi} /\left(y_{\xi}+\bar{h}\right)\right)(t, \xi)$ is a decreasing function, and $\left(U_{\xi} /\left(y_{\xi}+\bar{h}\right)\right)(t, \xi)$ is an increasing function, both with respect to time for $t \in\left[0, \min \left(\tau_{1}(\xi), T\right)\right]$. Therefore, we have

$$
\frac{U_{0, \xi}}{y_{0, \xi}+\bar{h}_{0}}(\xi) \leqslant \frac{U_{\xi}}{y_{\xi}+\bar{h}}(t, \xi) \leqslant 0 \quad \text { and } \quad 0 \leqslant \frac{y_{\xi}}{y_{\xi}+\bar{h}}(t, \xi) \leqslant \frac{y_{0, \xi}}{y_{0, \xi}+\bar{h}_{0}}(\xi) \text {, }
$$

for $t \in\left[0, \min \left(\tau_{1}(\xi), T\right)\right]$. In addition, for $\gamma$ sufficiently small, depending only on $M$ and $T$, we have

$$
\kappa_{1-\gamma} \subset\left\{\xi \in \mathbb{R} \mid 0 \leqslant \tau_{1}(\xi)<T\right\} .
$$


(iii) Moreover, for any given $\gamma>0$, there exists $\hat{T}>0$ such that

$$
\left\{\xi \in \mathbb{R} \mid 0<\tau_{1}(\xi)<\hat{T}\right\} \subset \kappa_{1-\gamma} .
$$

Although we have now constructed a new class of solutions in Lagrangian coordinates, there is one more fact we want to point out. The construction of $\alpha$-dissipative solutions involves the sequence of breaking times $\left\{\tau_{j}(\xi)\right\}$ for every point $\xi$. At first sight, it is not clear that this possibly infinite sequence does not accumulate.

Corollary 19. Denote by $\Theta(t)=\left(y, U, y_{\xi}, U_{\xi}, \bar{h}, h, r\right)(t)$ the global solution of (32) with $\Theta(0)=\Theta_{0} \in \mathcal{G} \cap B_{M}$ in $C\left(\mathbb{R}_{+}, \mathcal{G}\right)$. For any $\xi \in \mathbb{R}$, the possibly infinite sequence $\tau_{j}(\xi)$ cannot accumulate.

In particular, there exists a time $\hat{T}$ depending on $M$ such that any point $\xi$ can experience wave breaking at most once within the time interval $\left[T_{0}, T_{0}+\hat{T}\right]$ for any $T_{0} \geqslant 0$. More precisely, given $\xi \in \mathbb{R}$, we have

$$
\tau_{j+1}(\xi)-\tau_{j}(\xi)>\hat{T} \quad \text { for all } j .
$$

In addition, for $\hat{T}$ sufficiently small, we get that in this case $U_{\xi}(t, \xi) \geqslant 0$ for all $t \in\left[\tau_{j}(\xi), \tau_{j}(\xi)+\hat{T}\right]$.

Proof. In the proof of Lemma 16, we showed that

$$
\|P(\Theta)\|_{L_{\infty}^{\infty} L^{\infty}}+\|Q(\Theta)\|_{L_{\infty}^{\infty} L^{\infty}}+\|U\|_{L_{\infty}^{\infty} L^{\infty}}^{2} \leqslant 5 \Sigma(0),
$$

where $L_{\infty}^{\infty}=L_{T=\infty}^{\infty}$. This means, in particular, that the constant $C(\bar{M})$ in the proof of Lemma 12, for the global solution, can be chosen to be independent of time. Thus we can conclude from Lemma 12 that there exists a constant $\hat{T}$ such that $\tau_{j+1}(\xi)-\tau_{j}(\xi)>\hat{T}$ for all $j$.

\section{From Eulerian to Lagrangian variables and vice versa}

Let us define in detail our variables in Eulerian coordinates. As explained in the introduction, the energy distribution can concentrate, and therefore our set of Eulerian variables contains not only the functions $u(t)$ and $\rho(t)$ but also a measure $\mu(t)$, which properly describes the concentrated amount of energy at breaking times. This measure $\mu(t)$, which describes only part of the energy in general, is treated as an independent variable, but still remains strongly connected to $u(t)$ and $\rho(t)$ through its absolutely continuous part; see (168) below. In addition, in order to enable the construction of the semigroup, we add to the set of Eulerian variables 
the measure $v(t)$, which allows us, together with $\mu(t)$, to determine how much energy has been dissipated. For the solution we construct (see Section 4), the measure $\mu(t)$ is in general discontinuous in time, while $v(t)$ remains continuous.

DEFINITION 4 (Eulerian coordinates). The set $\mathcal{D}$ is composed of all $(u, \rho, \mu, v)$ such that the following hold:

(i) $u \in H^{1}(\mathbb{R})$;

(ii) $\rho \in L^{2}(\mathbb{R})$;

(iii) $\mu$ is a positive finite Radon measure whose absolutely continuous part, $\mu_{\mathrm{ac}}$, satisfies

$$
\mu_{\mathrm{ac}}=\left(u_{x}^{2}+\rho^{2}\right) d x
$$

(iv) $v$ is a positive finite Radon measure such that $\mu \leqslant v$.

Note that $\mu \leqslant v$ implies that $\mu$ is absolutely continuous with respect to $\nu$, and therefore there exists a measurable function $f$ such that

$$
\mu=f \nu \quad \text { and } \quad 0 \leqslant f \leqslant 1 .
$$

REMARK 20. At first sight, it might seem surprising that we need two measures to be able to construct a semigroup of solutions, but both of them play an essential role.

The measure $\mu$, on the one hand, describes the concentrated amount of energy at breaking times, and is therefore, in general, discontinuous with respect to time. Moreover, it helps to measure the total energy $E(t)$ at any time, since

$$
E(t)=\int_{\mathbb{R}} u^{2}(t, x) d x+\mu(t, \mathbb{R}) .
$$

Thus also the energy is in general a discontinuous function, and, in particular, it drops suddenly at breaking times if $\alpha \neq 0$, while it is preserved for all times in the conservative case.

The measure $v$, on the other hand, is continuous with respect to time, and plays a key role when identifying equivalence classes. Moreover, it enables us to determine how much energy has dissipated from the system up to a certain time, since

$$
\int_{\mathbb{R}} u^{2}(t, x) d x+v(t, \mathbb{R})
$$

is independent of time. 
For conservative solutions, no energy vanishes from the system, and therefore it is natural to impose that $\mu=v$. In the case of dissipative solutions, all the energy that concentrates at isolated points where wave breaking takes place vanishes from the system. The measure $\mu$, which corresponds to the energy, is purely absolutely continuous, while $v-\mu$ describes how much energy we already lost. If $\alpha \in(0,1]$, we can initially choose the two measures to be equal, $v_{0}=\mu_{0}$, but, as soon as wave breaking takes place, they will differ. In particular, $v$ does not coincide with the measure $\mu_{\text {cons }}$ for conservative solutions.

DEFINITION 5 (Relabeling functions). We denote by $G$ the subgroup of the group of homeomorphisms from $\mathbb{R}$ to $\mathbb{R}$ such that

$$
\begin{gathered}
f-\text { Id and } f^{-1}-\text { Id both belong to } W^{1, \infty}(\mathbb{R}), \\
f_{\xi}-1 \text { belongs to } L^{2}(\mathbb{R}),
\end{gathered}
$$

where Id denotes the identity function. Given $\kappa \geqslant 0$, we denote by $G_{\kappa}$ the subset $G_{\kappa}$ of $G$ defined by

$$
G_{\kappa}=\left\{f \in G \mid\|f-\mathrm{Id}\|_{W^{1, \infty}}+\left\|f^{-1}-\operatorname{Id}\right\|_{W^{1, \infty}} \leqslant \kappa\right\} .
$$

Note that, for $\kappa=0$, the set $G_{0}$ reduces to one element, the identity; that is,

$$
G_{0}=\{\mathrm{Id}\} .
$$

Definition 6 (Lagrangian coordinates). The subsets $\mathcal{F}$ and $\mathcal{F}_{\kappa}$ of $\mathcal{G}$ are defined as

$$
\mathcal{F}_{\kappa}=\left\{\Theta=\left(y, U, y_{\xi}, U_{\xi}, \bar{h}, h, r\right) \in \mathcal{G} \mid y+H \in G_{\kappa}\right\},
$$

and

$$
\mathcal{F}=\left\{\Theta=\left(y, U, y_{\xi}, U_{\xi}, \bar{h}, h, r\right) \in \mathcal{G} \mid y+H \in G\right\},
$$

where $H(t, \xi)$ is defined by

$$
H(t, \xi)=\int_{-\infty}^{\xi} h(t, \tilde{\xi}) d \tilde{\xi}
$$

In addition, it should be pointed out that the condition on $y+H$ is closely linked to $\left\|1 /\left(y_{\xi}+h\right)\right\|_{L^{\infty}}$, as the following lemma shows.

LEMMA 21 [27, Lemma 3.2]. Let $\kappa \geqslant 0$. If $f$ belongs to $G_{\kappa}$, then $1 /(1+\kappa) \leqslant$ $f_{\xi} \leqslant 1+\kappa$ almost everywhere. Conversely, if $f$ is absolutely continuous, $f-\operatorname{Id} \in$ $W^{1, \infty}(\mathbb{R}), f$ satisfies $(172 b)$, and there exists $d \geqslant 1$ such that $1 / d \leqslant f_{\xi} \leqslant d$ almost everywhere, then $f \in G_{\kappa}$ for some $\kappa$ depending only on $d$ and $\| f-$ Id $\|_{W^{1, \infty}}$. 
An immediate consequence of $(37 f)$ is therefore the following result.

LEMMA 22. The space $\mathcal{G}$ is preserved by the governing equations (32).

For the sake of simplicity, for any $\Theta=\left(y, U, y_{\xi}, U_{\xi}, \bar{h}, h, r\right) \in \mathcal{F}$ and any function $f \in G$, we denote $\left(y \circ f, U \circ f, y_{\xi} \circ f f_{\xi}, U_{\xi} \circ f f_{\xi}, \bar{h} \circ f f_{\xi}, h \circ f f_{\xi}\right.$, $r \circ f f_{\xi}$ ) by $\Theta \circ f$.

Proposition 23. The map from $G \times \mathcal{F}$ to $\mathcal{F}$ given by $(f, \Theta) \mapsto \Theta \circ f$ defines an action of the group $G$ on $\mathcal{F}$.

Since $G$ is acting on $\mathcal{F}$, we can consider the quotient space $\mathcal{F} / G$ of $\mathcal{F}$ with respect to the action of the group $G$. The equivalence relation on $\mathcal{F}$ is defined as follows: for any $\Theta, \Theta^{\prime} \in \mathcal{F}$, we say that $\Theta$ and $\Theta^{\prime}$ are equivalent if there exists a relabeling function $f \in G$ such that $\Theta^{\prime}=\Theta \circ f$. We denote by $\Pi(\Theta)=[\Theta]$ the projection of $\mathcal{F}$ into the quotient space $\mathcal{F} / G$, and introduce the mapping $\Lambda: \mathcal{F} \rightarrow \mathcal{F}_{0}$ given by

$$
\Lambda(\Theta)=\Theta \circ(y+H)^{-1}
$$

for any $\Theta=\left(y, U, y_{\xi}, U_{\xi}, \bar{h}, h, r\right) \in \mathcal{F}$. We have $\Lambda(\Theta)=\Theta$ when $\Theta \in \mathcal{F}_{0}$. It is not hard to prove that $\Lambda$ is invariant under the $G$ action; that is, $\Lambda(\Theta \circ f)=\Lambda(\Theta)$ for any $\Theta \in \mathcal{F}$ and $f \in G$. Hence, there corresponds to $\Lambda$ a mapping $\tilde{\Lambda}$ from the quotient space $\mathcal{F} / G$ to $\mathcal{F}_{0}$ given by $\tilde{\Lambda}([\Theta])=\Lambda(\Theta)$, where $[\Theta] \in \mathcal{F} / G$ denotes the equivalence class of $\Theta \in \mathcal{F}$. For any $\Theta \in \mathcal{F}_{0}$, we have $\tilde{\Lambda} \circ \Pi(\Theta)=\Lambda(\Theta)=$ $\Theta$. Hence, $\left.\tilde{\Lambda} \circ \Pi\right|_{\mathcal{F}_{0}}=\left.\operatorname{Id}\right|_{\mathcal{F}_{0}}$.

Denote by $S: \mathcal{F} \times[0, \infty) \rightarrow \mathcal{F}$ the semigroup which to any initial data $\Theta_{0} \in \mathcal{F}$ associates the solution $\Theta(t)$ of the system of differential equations (32) at time $t$. As indicated earlier, the two-component Camassa-Holm system is invariant with respect to relabeling. More precisely, using our terminology, we have the following result.

TheOREM 24. For any $t>0$, the mapping $S_{t}: \mathcal{F} \rightarrow \mathcal{F}$ is G-equivariant; that is,

$$
S_{t}(\Theta \circ f)=S_{t}(\Theta) \circ f
$$

for any $\Theta \in \mathcal{F}$ and $f \in G$. Hence, the mapping $\tilde{S}_{t}$ from $\mathcal{F} / G$ to $\mathcal{F} / G$ given by

$$
\tilde{S}_{t}([\Theta])=\left[S_{t} \Theta\right]
$$

is well defined and generates a semigroup. 
We have the following diagram:

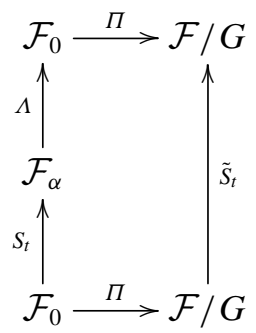

Next, we describe the correspondence between Eulerian coordinates (functions in $\mathcal{D}$ ) and Lagrangian coordinates (functions in $\mathcal{F} / G$ ). In order to do so, we have to take into account the facts that the set $\mathcal{D}$ allows the energy density to have a singular part and that a positive amount of energy can concentrate on a set of Lebesgue measure zero.

We first define the mapping $L$ from $\mathcal{D}$ to $\mathcal{F}$ which to any initial data in $\mathcal{D}$ associates an initial data for the equivalent system in $\mathcal{F}$.

Definition 7. For any $(u, \rho, \mu, v)$ in $\mathcal{D}$, let

$$
\begin{gathered}
y(\xi)=\sup \{y \mid v((-\infty, y))+y<\xi\}, \\
h(\xi)=1-y_{\xi}(\xi), \\
U(\xi)=u \circ y(\xi), \\
r(\xi)=\rho \circ y(\xi) y_{\xi}(\xi), \\
\bar{h}(\xi)=f \circ y(\xi) h(\xi),
\end{gathered}
$$

where $f$ is given through (169). Then $\left(y, U, y_{\xi}, U_{\xi}, \bar{h}, h, r\right) \in \mathcal{F}$. We denote by $L: \mathcal{D} \rightarrow \mathcal{F}$ the mapping which to any element $(u, \rho, \mu, v) \in \mathcal{D}$ associates $\Theta=\left(y, U, y_{\xi}, U_{\xi}, \bar{h}, h, r\right) \in \mathcal{F}$ given by (176).

Proof. (Well posedness of Definition 7) We have to prove that ( $y, U, y_{\xi}, U_{\xi}, \bar{h}, h$, $r) \in \mathcal{F}$. The proof follows the same lines as in [27, Theorem 3.8]. The properties $(37 a)-(37 f)$ are proved in the same way, and we do not reproduce the proofs here. It remains to prove $(37 g)$ and $(37 h)$. Since $f \leqslant 1$, see (169), we have that $\bar{h} \leqslant h$ follows from $(176 e)$. Let us prove $(37 g)$. First, we show that

$$
v=y_{\#}(h(\xi) d \xi) \quad \text { and } \quad \mu=y_{\#}(\bar{h}(\xi) d \xi) .
$$


For any given $x \in \mathbb{R}$, let us define $\xi$ as

$$
\xi=\sup \{\bar{\xi} \mid y(\bar{\xi})=x\}
$$

We know that $y$ is increasing and Lipschitz (we refer to [27]), so $y$ is continuous. Hence, $y(\xi)=x$. Moreover, by $(176 a)$ and (176b), the definitions of $y$ and $h$, we have for $\xi \in \mathbb{R}$ that

$$
y(\xi)=\sup \{y \mid v((-\infty, y))+y<\xi\}
$$

and

$$
y(\xi)+\int_{-\infty}^{\xi} h(\bar{\xi}) d \bar{\xi}=\xi .
$$

Thus we have, for any $\bar{y}>y(\xi)$,

$$
y(\xi)+\int_{-\infty}^{\xi} h(\bar{\xi}) d \bar{\xi} \leqslant v((-\infty, \bar{y}))+\bar{y} .
$$

Letting $\bar{y}$ tend to $y(\xi)=x$ then yields

$$
\int_{-\infty}^{\xi} h(\bar{\xi}) d \bar{\xi} \leqslant v((-\infty, x]) .
$$

For any $\varepsilon>0$, by the definition of $\xi$, we have that $y(\xi+\varepsilon)>y(\xi)$. Hence, following the same lines as before, we get

$$
y(\xi)+v((-\infty, y(\xi)]) \leqslant y(\xi+\varepsilon)+\int_{-\infty}^{\xi+\varepsilon} h(\bar{\xi}) d \bar{\xi}
$$

which, after letting $\varepsilon$ tend to zero, yields

$$
v((-\infty, x]) \leqslant \int_{-\infty}^{\xi} h(\bar{\xi}) d \bar{\xi} .
$$

Combining (181), (182), and the definition of $\xi$, we get

$$
v((-\infty, x])=\int_{y^{-1}((-\infty, x])} h(\bar{\xi}) d \bar{\xi},
$$

which proves the first identity in (177). Let us prove the second one. For any Borel set $A$, we have

$$
\mu(A)=\int_{A} f d v=\int_{y^{-1}(A)} f(y(\xi)) h(\xi) d \xi,
$$


because $v=y_{\#}(h(\xi) d \xi)$. Then, using (176e), we get $\mu(A)=\int_{y^{-1}(A)} \bar{h}(\xi) d \xi$, which concludes the proof of (177). We introduce the sets

$$
B=\left\{\begin{array}{l|l}
x \in \mathbb{R} & \lim _{\delta \rightarrow 0} \frac{1}{2 \delta} \mu(x-\delta, x+\delta)=\left(u_{x}^{2}+\rho^{2}\right)(x)
\end{array}\right\}
$$

and

$$
A=\left\{\xi \in y^{-1}(B) \mid y_{\xi}(\xi)>0\right\} .
$$

From Besicovitch's derivation theorem [1], we have meas $\left(B^{c}\right)=0$. For almost every $\xi \in A$, we denote $x=y(\xi)$, and define $\xi_{-}^{\delta}$ and $\xi_{+}^{\delta}$ as

$$
\xi_{-}^{\delta}=\sup \{\bar{\xi} \mid y(\bar{\xi})=x-\delta\} \quad \text { and } \quad \xi_{+}^{\delta}=\inf \{\bar{\xi} \mid y(\bar{\xi})=x+\delta\},
$$

for any $\delta>0$. The continuity of $y$ implies that $y\left(\xi_{-}^{\delta}\right)=x-\delta$ and $y\left(\xi_{+}^{\delta}\right)=x+\delta$. From (177), we obtain

$$
\mu(x-\delta, x+\delta)=\int_{\xi_{-}^{\delta}}^{\xi_{+}^{\delta}} \bar{h}(\bar{\xi}) d \bar{\xi},
$$

as Definition (183) implies that $y^{-1}((x-\delta, x+\delta))=\left(\xi_{-}^{\delta}, \xi_{+}^{\delta}\right)$. Since $y_{\xi}(\xi)>0$, we have $\xi_{-}^{\delta}<\xi_{+}^{\delta}, \lim _{\delta \rightarrow 0} \xi_{+}^{\delta}=\lim _{\delta \rightarrow 0} \xi_{+}^{\delta}=\xi$, and

$$
\frac{1}{2 \delta} \mu(x-\delta, x+\delta)=\frac{\int_{\xi_{-}^{\delta}}^{\xi_{+}^{\delta}} \bar{h}(\bar{\xi}) d \bar{\xi}}{\xi_{+}^{\delta}-\xi_{-}^{\delta}} \frac{\xi_{+}^{\delta}-\xi_{-}^{\delta}}{\int_{\xi_{-}^{\delta}}^{\xi_{+}^{\delta}} y_{\xi}(\bar{\xi}) d \bar{\xi}} .
$$

Letting $\delta$ tend to zero, we get

$$
u_{x}^{2}(y(\xi))+\rho^{2}(y(\xi))=\frac{\bar{h}(\xi)}{y_{\xi}(\xi)} .
$$

As $U_{\xi}=u_{x} \circ y y_{\xi}$ and $r=\rho \circ y y_{\xi}$ almost everywhere, we obtain that

$$
y_{\xi} \bar{h}=U_{\xi}^{2}+r^{2},
$$

for almost every $\xi \in A$. However, as meas $\left(B^{c}\right)=0$, we can prove that meas $\left(\left\{\xi \in \mathbb{R} \mid y_{\xi}(\xi)>0\right.\right.$ and $\left.\left.y(\xi) \in B^{c}\right\}\right)=0$, see [27, Lemma 3.9], and therefore (184) holds also for almost every $\xi \in \mathbb{R}$ such that $y_{\xi}(\xi)>0$.

It is left to show that (184) is also true for almost all $\xi$ such that $y_{\xi}(\xi)=0$. Following closely the proof of [27, Theorem 3.8], one obtains that the function

$$
\xi \mapsto \int_{-\infty}^{y(\xi)}\left(u_{x}^{2}+\rho^{2}\right) d x
$$


is Lipschitz continuous with Lipschitz constant at most one. Thus we have, for all $\xi, \tilde{\xi} \in \mathbb{R}$, using the Cauchy-Schwarz inequality,

$$
\begin{aligned}
|U(\tilde{\xi})-U(\xi)| & =\left|\int_{y(\xi)}^{y(\tilde{\xi})} u_{x} d x\right| \\
& \leqslant \sqrt{|y(\tilde{\xi})-y(\xi)|} \sqrt{\left|\int_{y(\xi)}^{y(\tilde{\xi})} u_{x}^{2} d x\right|} \\
& \leqslant \sqrt{|y(\tilde{\xi})-y(\xi)|} \sqrt{\left|\int_{y(\xi)}^{y(\tilde{\xi})} u_{x}^{2}+\rho^{2} d x\right|} \\
& \leqslant|\xi-\tilde{\xi}|,
\end{aligned}
$$

because $y$ and $\xi \mapsto \int_{-\infty}^{y(\xi)}\left(u_{x}^{2}+\rho^{2}\right) d x$ are Lipschitz with Lipschitz constant at most one. Hence, $U$ is Lipschitz and therefore differentiable almost everywhere. Let

$$
B_{2}=\left\{x \in B \mid \lim _{\delta \rightarrow 0} \frac{1}{\delta} \int_{x-\delta}^{x+\delta} u_{x}(s) d s=u_{x}(x)\right\} .
$$

From Besicovitch's derivation theorem, we have that $\left\{\xi \mid y_{\xi}(\xi)=0\right\} \subset B_{2}^{c}$ and meas $\left(B_{2}^{c}\right)=0$. Then (186) implies that

$$
\left|\frac{U(\tilde{\xi})-U(\xi)}{\tilde{\xi}-\xi}\right| \leqslant \sqrt{\frac{y(\tilde{\xi})-y(\xi)}{\tilde{\xi}-\xi}},
$$

due to the Lipschitz continuity with Lipschitz constant of at most one of $y$ and $\xi \mapsto \int_{-\infty}^{y(\xi)}\left(u_{x}^{2}+\rho^{2}\right) d x$. Hence, for almost every $\xi$ in $y^{-1}\left(B_{2}^{c}\right)$, we have

$$
\left|U_{\xi}(\xi)\right| \leqslant \sqrt{y_{\xi}(\xi)}
$$

A similar argument yields that

$$
|r(\xi)| \leqslant \sqrt{y_{\xi}(\xi)}
$$

Since meas $\left(B_{2}^{c}\right)=0$, we have, by [27, Lemma 3.9], that $y_{\xi}=0$ almost everywhere on $y^{-1}\left(B_{2}^{c}\right)$. Hence $U_{\xi}=0$ and $r=0$ almost everywhere on $y^{-1}\left(B_{2}^{c}\right)$. Thus $y_{\xi} \bar{h}=$ $U_{\xi}^{2}+r^{2}$ almost everywhere on $y^{-1}\left(B_{2}^{c}\right)$, which is (184). This finishes the proof of $(37 h)$.

In fact, $L$ is a mapping from $\mathcal{D}$ to the set $\mathcal{F}_{0} \subset \mathcal{F}$, which contains exactly one element of each equivalence class.

On the other hand, to any element in $\mathcal{F}$ there corresponds a unique element in $\mathcal{D}$ which is given by the mapping $M$ defined below. 
Definition 8. Take any element $\Theta=\left(y, U, y_{\xi}, U_{\xi}, \bar{h}, h, r\right) \in \mathcal{F}$. Then, the measure $y_{\#}(r(\xi) d \xi)$ is absolutely continuous, and we define $(u, \rho, \mu, v)$ as follows:

$$
\begin{gathered}
u(x)=U(\xi) \quad \text { for any } \xi \text { such that } x=y(\xi), \\
\mu=y_{\#}(\bar{h}(\xi) d \xi), \\
v=y_{\#}(h(\xi) d \xi), \\
\rho(x) d x=y_{\#}(r(\xi) d \xi) .
\end{gathered}
$$

We have that $(u, \rho, \mu, v)$ belongs to $\mathcal{D}$. We denote by $M: \mathcal{F} \rightarrow \mathcal{D}$ the mapping which to any $\Theta$ in $\mathcal{F}$ associates the element $(u, \rho, \mu, v) \in \mathcal{D}$ as given by (191). In particular, the mapping $M$ is invariant under relabeling.

Finally, we identify the connection between the equivalence classes in Lagrangian coordinates and the set of Eulerian coordinates. The proof is similar to the one found in [27], and we do not reproduce it here.

THEOREM 25. The mappings $M$ and L are invertible. We have

$$
L \circ M=\operatorname{Id}_{\mathcal{F} / G} \quad \text { and } \quad M \circ L=\operatorname{Id}_{\mathcal{D}} .
$$

\section{Semigroup of solutions}

In the previous section, we defined the connection between Eulerian and Lagrangian coordinates, which is the main tool when defining weak solutions of the $2 \mathrm{CH}$ system. The aim of this section is to show that we obtained a semigroup of solutions. Accordingly, we define $T_{t}$ as

$$
T_{t}=M \circ S_{t} \circ L \text {. }
$$

Definition 9. Assume that $u:[0, \infty) \times \mathbb{R} \rightarrow \mathbb{R}$ and $\rho:[0, \infty) \times \mathbb{R} \rightarrow \mathbb{R}$ satisfy the following.

(i) $u \in L^{\infty}\left([0, \infty), H^{1}(\mathbb{R})\right)$, and $\rho \in L^{\infty}\left([0, \infty), L^{2}(\mathbb{R})\right)$,

(ii) the equations

$$
\begin{aligned}
& \iint_{[0, \infty) \times \mathbb{R}}\left[-u(t, x) \phi_{t}(t, x)+\left(u(t, x) u_{x}(t, x)+P_{x}(t, x)\right) \phi(t, x)\right] d x d t \\
& \quad=\int_{\mathbb{R}} u(0, x) \phi(0, x) d x \\
& \iint_{[0, \infty) \times \mathbb{R}}\left[\left(P(t, x)-u^{2}(t, x)-\frac{1}{2} u_{x}^{2}(t, x)-\frac{1}{2} \rho^{2}(t, x)\right) \phi(t, x)\right.
\end{aligned}
$$




$$
\left.+P_{x}(t, x) \phi_{x}(t, x)\right] d x d t=0
$$

and

$$
\begin{aligned}
& \iint_{[0, \infty) \times \mathbb{R}}\left[-\rho(t, x) \phi_{t}(t, x)-u(t, x) \rho(t, x) \phi_{x}(t, x)\right] d x d t \\
& \quad=\int_{\mathbb{R}} \rho(0, x) \phi(0, x) d x
\end{aligned}
$$

hold for all $\phi \in C_{0}^{\infty}([0, \infty) \times \mathbb{R})$. Then we say that $(u, \rho)$ is a weak global solution of the two-component Camassa-Holm system.

THEOREM 26. The mapping $T_{t}$ is a semigroup of solutions of the $2 C H$ system. Given some initial data $\left(u_{0}, \rho_{0}, \mu_{0}, v_{0}\right) \in \mathcal{D}$, let $(u(t, \cdot), \rho(t, \cdot), \mu(t, \cdot), v(t, \cdot))=$ $T_{t}\left(u_{0}, \rho_{0}, \mu_{0}, v_{0}\right)$. Then $(u, \rho)$ is a weak solution to $(14)$, and $(u, \rho, \mu)$ is a weak solution to

$$
\left(u^{2}+\mu\right)_{t}+\left(u\left(u^{2}+\mu\right)\right)_{x} \leqslant\left(u^{3}-2 P u\right)_{x} .
$$

The function

$$
F(t)=\int_{\mathbb{R}} d(\nu(t, x)-\mu(t, x))-\int_{\mathbb{R}} d(v(0, x)-\mu(0, x)),
$$

which is an increasing semicontinuous function, equals the amount of energy that has vanished from the solution up to time $t$.

Proof. This proof follows essentially the same lines as the one in [18] and of the proof of (196), which we present here. Let $\phi \in C_{0}^{\infty}((0, \infty) \times \mathbb{R})$ such that $\phi(t, x) \geqslant 0$. Since $U(t, \xi)$ is continuous with respect to time, we have

$$
\begin{aligned}
& \iint_{\mathbb{R}_{+} \times \mathbb{R}} u^{2} \phi_{t}(t, x) d x d t \\
& =-\iint_{\mathbb{R}_{+} \times \mathbb{R}}\left(U^{2}(t, \xi) U_{\xi}(t, \xi)-2 U(t, \xi) Q(t, \xi) y_{\xi}(t, \xi)\right) \phi(t, y(t, \xi)) d \xi d t \\
& \quad-\iint_{\mathbb{R}_{+} \times \mathbb{R}} U^{3}(t, \xi) \phi_{\xi}(t, y(t, \xi)) d \xi d t .
\end{aligned}
$$

The measure $\mu$ in Eulerian coordinates corresponds to the function $\bar{h}(t, \xi)$, which is discontinuous with respect to time, in Lagrangian coordinates. Thus it is important for the following calculations to keep in mind that $\bar{h}(t, \xi)$ can be rewritten as $h(t, \xi)=\bar{h}(t, \xi)+\sum_{j=0}^{\infty} \chi_{\left\{\tau_{j}(\xi) \leqslant t\right\}}(\xi) l_{j}(\xi)$, where $h(t, \xi)$ is continuous with respect to time and corresponds to the measure $v$ in Eulerian 
coordinates, by Definition 8 and $\tau_{0}(\xi)=0$. Thus

$$
\begin{aligned}
& \iint_{\mathbb{R}_{+} \times \mathbb{R}} \phi_{t}(t, x) d \mu(t, x) d t \\
& =\iint_{\mathbb{R}_{+} \times \mathbb{R}} \phi_{t}(t, y(t, \xi)) \bar{h}(t, \xi) d \xi d t \\
& =\iint_{\mathbb{R}_{+} \times \mathbb{R}}\left[(\phi(t, y(t, \xi)))_{t}-\phi_{x}(t, y(t, \xi)) y_{t}(t, \xi)\right] \bar{h}(t, \xi) d \xi d t \\
& =\iint_{\mathbb{R}_{+} \times \mathbb{R}}(\phi(t, y(t, \xi)))_{t} \bar{h}(t, \xi) d t d \xi \\
& -\iint_{\mathbb{R}_{+} \times \mathbb{R}} U(t, \xi) \bar{h}(t, \xi) \phi_{x}(t, y(t, \xi)) d \xi d t \\
& =\iint_{\mathbb{R}_{+} \times \mathbb{R}}(\phi(t, y(t, \xi)))_{t} h(t, \xi) d t d \xi \\
& -\iint_{\mathbb{R}_{+} \times \mathbb{R}}(\phi(t, y(t, \xi)))_{t} \sum_{j=0}^{\infty} \chi_{\left\{\tau_{j}(\xi) \leqslant t\right\}}(\xi) l_{j}(\xi) d t d \xi \\
& -\iint_{\mathbb{R}_{+} \times \mathbb{R}} U(t, \xi) \bar{h}(t, \xi) \phi_{x}(t, y(t, \xi)) d \xi d t \\
& =-\iint_{\mathbb{R}_{+} \times \mathbb{R}} 2\left(U^{2}(t, \xi)-P(t, \xi)\right) U_{\xi}(t, \xi) \phi(t, y(t, \xi)) d \xi d t \\
& -\iint_{\mathbb{R}_{+} \times \mathbb{R}} U(t, \xi) \bar{h}(t, \xi) \phi_{x}(t, y(t, \xi)) d \xi d t \\
& +\int_{\mathbb{R}} \sum_{j=1}^{\infty} \phi\left(\tau_{j}(\xi), y\left(\tau_{j}(\xi), \xi\right)\right) l_{j}(\xi) d \xi .
\end{aligned}
$$

Note that the integral in the seventh line is well defined since, by construction, $0 \leqslant$ $\sum_{j=0}^{\infty} \chi_{\left\{\tau_{j}(\xi) \leqslant t\right\}}(\xi) l_{j}(\xi) \leqslant h(t, \xi)$, and therefore the integrand belongs to $L^{1}(\mathbb{R})$ for each fixed $t$. In addition, $\phi \in C_{0}^{\infty}((0, \infty) \times \mathbb{R})$, and hence this integral exists. Similar conclusions hold for the integral with respect to $\xi$ in the last line.

Observe that we have

$$
\begin{aligned}
& \iint_{\mathbb{R}_{+} \times \mathbb{R}}\left(2 P U_{\xi}+2 U Q y_{\xi}-3 U^{2} U_{\xi}\right)(t, \xi) \phi(t, y(t, \xi)) d \xi d t \\
& =-\iint_{\mathbb{R}_{+} \times \mathbb{R}}\left(2 P U-U^{3}\right)(t, \xi) \phi_{\xi}(t, y(t, \xi)) d \xi d t
\end{aligned}
$$


Gathering (198), (199), and applying (200) yields

$$
\begin{aligned}
& \iint_{\mathbb{R}_{+} \times \mathbb{R}} u^{2} \phi_{t}(t, x) d x d t+\iint_{\mathbb{R}_{+} \times \mathbb{R}} \phi_{t}(t, x) d \mu(t, x) d t \\
& =-\iint_{\mathbb{R}_{+} \times \mathbb{R}} 2 P(t, x) u(t, x) \phi_{x}(t, x) d t d x \\
& \quad-\iint_{\mathbb{R}_{+} \times \mathbb{R}} u(t, x) \phi_{x}(t, x) d \mu(t, x) d t \\
& \quad+\int \sum_{\mathbb{R}}^{\infty} \phi\left(\tau_{j}(\xi), y\left(\tau_{j}(\xi), \xi\right)\right) l_{j}(\xi) d \xi .
\end{aligned}
$$

The integral in the last line is finite and positive, and hence we have proved (196).

We have now shown that this new solution concept yields global weak solutions of the $2 \mathrm{CH}$ system. However, there is one more question which is of great interest. Recall that $(u, \rho)$ satisfies the same equation, namely (14), independently of the value $\alpha$, yet we have carefully constructed a solution for a given $\alpha$. One can turn this around and ask: given a solution $(u, \rho)$, can we determine $\alpha$ ? The answer is contained in the following theorem.

THEOREM 27. Let $(u, \rho, \mu, v)$ be a weak solution of the 2 CH system. The limits from the future and the past of the measure $\mu$ exist for all times, and we denote them as follows:

$$
\mu^{-}(t)=\lim _{t^{\prime} \uparrow t} \mu\left(t^{\prime}\right) \quad \text { and } \quad \mu^{+}(t)=\lim _{t^{\prime} \downarrow t} \mu\left(t^{\prime}\right) .
$$

We have that the measure $\mu$ is continuous backward in time; that is,

$$
\mu^{+}=\mu
$$

for all $t$. In the other direction, going forward in time, we have that

$$
\mu=\mu_{\mathrm{ac}}^{-}+(1-\alpha) \mu_{\mathrm{s}}^{-},
$$

for all $t$; that is,

$$
\mu_{\mathrm{ac}}=\mu_{\mathrm{ac}}^{-} \quad \text { and } \quad \mu_{\mathrm{s}}=(1-\alpha) \mu_{\mathrm{s}}^{-} .
$$

Moreover, we have that, for almost every time $t$,

$$
\mu^{+}(t)=\mu^{-}(t)=\mu(t)=\mu_{\mathrm{ac}}(t) .
$$


Proof. We prove the theorem first for $\alpha<1$. Given $t \in \mathbb{R}_{+}$, we define

$$
\tilde{\bar{h}}(t, \xi)= \begin{cases}\frac{1}{1-\alpha} \bar{h}(t, \xi) & \text { if } y_{\xi}(t, \xi)=0 \\ \bar{h}(t, \xi) & \text { otherwise. }\end{cases}
$$

We claim that, for almost every $\xi$,

$$
\bar{h}(t-0, \xi)=\lim _{t^{\prime} \uparrow t} \bar{h}\left(t^{\prime}, \xi\right)=\tilde{\bar{h}}(t, \xi) \quad \text { and } \quad \lim _{t^{\prime} \downarrow t} \bar{h}\left(t^{\prime}, \xi\right)=\bar{h}(t, \xi) .
$$

Indeed, if $y_{\xi}(t, \xi)>0$, then $\tau_{n}(\xi)<t<\tau_{n+1}(\xi)$, and $\bar{h}\left(t^{\prime}, \xi\right)$ is differentiable in $t^{\prime}$. It is therefore continuous, and we have

$$
\lim _{t^{\prime} \uparrow t} \bar{h}\left(t^{\prime}, \xi\right)=\lim _{t^{\prime} \downarrow t} \bar{h}\left(t^{\prime}, \xi\right)=\bar{h}(t, \xi) .
$$

If $y_{\xi}(t, \xi)=0$, there exists $n$ such that $t=\tau_{n}(\xi)$. In $\left[\tau_{n-1}(\xi), \tau_{n}(\xi)\right)$, the function $t^{\prime} \mapsto \bar{h}\left(t^{\prime}, \xi\right)$ satisfies the ordinary differential equation (32f). Hence, $\lim _{t^{\prime} \uparrow t} \bar{h}\left(t^{\prime}, \xi\right)$ exists, and, by the jump conditions (32h), (32i), we have $\tilde{\bar{h}}(t-0$, $\xi)=\lim _{t^{\prime} \uparrow t} \bar{h}\left(t^{\prime}, \xi\right)=(1 /(1-\alpha)) \bar{h}\left(\tau_{n}(\xi), \xi\right)$. For $t^{\prime} \in\left[\tau_{n}(\xi), \tau_{n+1}(\xi)\right), \bar{h}\left(t^{\prime}, \xi\right)$ also satisfies $(32 f)$, and we then directly have $\lim _{t^{\prime} \downarrow} \bar{h}\left(t^{\prime}, \xi\right)=\bar{h}\left(\tau_{n}(\xi), \xi\right)$. This concludes the proof of (204). Let us now define the measure $\mu^{-}$as

$$
\mu^{-}(t)=y_{\#}(\bar{h}(t-0) d \xi) .
$$

We claim that

$$
\lim _{t^{\prime} \uparrow t} \mu\left(t^{\prime}\right)=\mu^{-}(t) \quad \text { and } \quad \lim _{t^{\prime} \downarrow t} \mu\left(t^{\prime}\right)=\mu(t) .
$$

Here, we use the weak star topology for the measure. For any continuous function $\phi \in C(\mathbb{R})$ with compact support, we have

$$
\int_{\mathbb{R}} \phi(x) d \mu\left(t^{\prime}, x\right)=\int_{\mathbb{R}} \phi\left(y\left(t^{\prime}, \xi\right)\right) \bar{h}\left(t^{\prime}, \xi\right) d \xi .
$$

For almost every given $\xi$, we have that $\lim _{t^{\prime} \rightarrow t} y\left(t^{\prime}, \xi\right)=y(t, \xi)$, and, from (204), we have $\lim _{t^{\prime} \uparrow t} \bar{h}\left(t^{\prime}, \xi\right)=\tilde{\bar{h}}(t, \xi)$. Hence, the integrand in (207) tends to $\phi(y(t$, $\xi)) \tilde{\bar{h}}(t, \xi)$ when $t^{\prime}$ converges to $t$ from below. Moreover, since $\|y(t, \cdot)-\operatorname{Id}\|_{L^{\infty}}$ is bounded and $\phi$ has compact support, we can restrict the integration domain in (207) to a bounded domain. Then, the first proposition in (206) follows from the Lebesgue dominated convergence theorem applied to (207) by letting $t^{\prime}$ tend to $t$. The second proposition is proved in a similar way. Let us define

$$
B=\left\{\xi \in \mathbb{R} \mid y_{\xi}(t, \xi)>0\right\}
$$


and $A=y(t, B)$. Let us prove that

$$
\mu_{\mathrm{ac}}^{-}(t)=\left.\mu^{-}\right|_{A}(t) \quad \text { and } \quad \mu_{\mathrm{s}}^{-}(t)=\left.\mu^{-}\right|_{A^{c}}(t) .
$$

Here, $\left.\mu\right|_{A}(t)$ denotes the restriction of $\mu(t)$ to $A$; that is, $\left.\mu\right|_{A}(t, E)=\mu(t, E \cap$ $A$ ) for any Borel set $E$. We have meas $\left(A^{c}\right)=0$. Indeed, since $y$ is surjective, $A^{c} \subset y\left(t, B^{c}\right)$ and meas $\left(y\left(t, B^{c}\right)\right)=\int_{B^{c}} y_{\xi}(t, \xi) d \xi=0$, from the definition of $B$. Let us prove that $\left.\mu^{-}\right|_{A}(t)$ is absolutely continuous. We consider a set $E$ of zero measure. We have

$$
\left.\mu^{-}\right|_{A}(t, E)=\int_{y^{-1}(t, A \cap E)} \bar{h}(t, \xi) d \xi .
$$

We define $K_{M}=\left\{\xi \in \mathbb{R} \mid(\bar{h}(t, \xi)) /\left(y_{\xi}(t, \xi)\right) \leqslant M\right\}$. Let us prove that $y_{\xi}(t$, $\xi)>0$ for almost every $\xi \in y^{-1}(t, A)$. Assume the opposite. Then, since $y$ is surjective, there exist $\bar{\xi} \in B^{c}$ and $\xi \in B$ such that $y(t, \xi)=y(t, \bar{\xi})$. Since $y$ is increasing, we have $y_{\xi}(t, \xi)=y_{\xi}(t, \bar{\xi})=0$, which is a contradiction to the fact that $\xi \in B$. Thus, the indicator function of the set $K_{M}$, which we denote $\chi_{K_{M}}$, converges to one, almost everywhere in $y^{-1}(t, A \cap E)$, as $M$ tends to infinity. We have

$$
\int_{y^{-1}(t, A \cap E)} \chi_{K_{M}}(\xi) \bar{h}(t, \xi) d \xi \leqslant M \int_{y^{-1}(t, A \cap E)} y_{\xi}(t, \xi) d \xi=M \operatorname{meas}(A \cap E)=0,
$$

and, by the monotone convergence theorem, it follows that $\int_{y^{-1}(t, A \cap E)} \bar{h}(t, \xi) d \xi=$ 0 . Let us now prove (201). We have, for any Borel set $E$,

$$
\begin{aligned}
\mu(t, E) & =\int_{y^{-1}(t, E)} \bar{h}(t, \xi) d \xi \\
& =\int_{y^{-1}(t, E) \cap B} \bar{h}(t, \xi) d \xi+\int_{y^{-1}(t, E) \cap B^{c}} \bar{h}(t, \xi) d \xi \\
& =\int_{y^{-1}(t, E \cap A)} \overline{\tilde{h}}(t, \xi) d \xi+\int_{y^{-1}\left(t, E \cap A^{c}\right)}(1-\alpha) \tilde{\bar{h}}(t, \xi) d \xi
\end{aligned}
$$

by Definition (203) of $\bar{h}(t-0)$ and the fact that $y^{-1}(t, A)=B$. Then,

$$
\begin{aligned}
\mu(t, E) & =\int_{y^{-1}(t, E \cap A)} \tilde{\bar{h}}(t, \xi) d \xi+(1-\alpha) \int_{y^{-1}\left(t, E \cap A^{c}\right)} \tilde{\bar{h}}(t, \xi) d \xi \\
& =\mu_{\mathrm{ac}}^{-}(t, E)+(1-\alpha) \mu_{\mathrm{s}}^{-}(t, E),
\end{aligned}
$$

by (209) and (205). This concludes the proof of the theorem for $\alpha<1$. In the case where $\alpha=1$, Definition (203) cannot be used. However, the $\operatorname{limit}_{t^{\prime} \uparrow t} \bar{h}\left(t^{\prime}, \xi\right)$ 
still exists, and we denote it by $\tilde{\bar{h}}(t, \xi)$. The rest of the proof is the same up to (210), which is replaced by

$$
\begin{aligned}
\mu(t, E) & =\int_{y^{-1}(t, E)} \bar{h}(t, \xi) d \xi \\
& =\int_{y^{-1}(t, E) \cap B} \bar{h}(t, \xi) d \xi+\int_{y^{-1}(t, E) \cap B^{c}} \bar{h}(t, \xi) d \xi \\
& =\int_{y^{-1}(t, E \cap A)} \tilde{\bar{h}}(t, \xi) d \xi,
\end{aligned}
$$

because, in the fully dissipative case $\alpha=1$, we have $\bar{h}(t, \xi)=0$ when $y_{\xi}(t$, $\xi)=0$. Then, we obtain that $\mu(t, E)=\mu_{\mathrm{ac}}^{-}(t, E)$. We turn to the proof of (202). Let us introduce the set

$$
\begin{gathered}
\mathcal{A}=\left\{t \in \mathbb{R}_{+} \mid \text {for almost every } \xi, \text { either } y_{\xi}(t, \xi)>0\right. \text { or } \\
\left.\left(y_{\xi}(t, \xi)=0 \text { and } \bar{h}(t-0, \xi)=0\right)\right\} .
\end{gathered}
$$

For $t \in \mathcal{A}$, using (209), we get

$$
\begin{aligned}
\mu_{\mathrm{s}}^{-}(t)(\mathbb{R}) & =\int_{y^{-1}\left(y(t, B)^{c}\right)} \bar{h}(t-0, \xi) d \xi \\
& =\int_{y^{-1}\left(y(t, B)^{c}\right) \cap B} \bar{h}(t-0, \xi) d \xi \\
& \leqslant \int_{B^{c} \cap B} \bar{h}(t-0, \xi) d \xi=0 .
\end{aligned}
$$

Thus, (202) will be proved once we have proved that $\mathcal{A}$ has full measure. For a given $\xi \in \mathbb{R}$, we know from Corollary 19 that the collision times do not accumulate. For $\alpha<1$, this means that $y_{\xi}(t, \xi)=0$ only at isolated times $t$. For $\alpha=1$, assuming that a collision occurs at the point $\xi$, we have $y_{\xi}(t, \xi)>0$ for $t<\tau_{1}(\xi), y_{\xi}(t, \xi)=0$ for $t \geqslant \tau_{1}(\xi)$, but $\bar{h}(t, \xi)=0$ for all $t \geqslant \tau_{1}(\xi)$. Hence, in both cases, we have

$$
\operatorname{meas}\left(\left\{t \in \mathbb{R}_{+} \mid y_{\xi}(t, \xi)=0 \text { and } \bar{h}(t-0, \xi)>0\right\}\right)=0 .
$$

Using Fubini's theorem, we get

$$
\begin{aligned}
& \int_{\mathbb{R}^{+}} \operatorname{meas}\left(\left\{\xi \in \mathbb{R} \mid y_{\xi}(t, \xi)=0 \text { and } \bar{h}(t-0, \xi)>0\right\}\right) d t \\
& \quad=\int_{\mathbb{R}} \operatorname{meas}\left(\left\{t \in \mathbb{R}_{+} \mid y_{\xi}(t, \xi)=0 \text { and } \bar{h}(t-0, \xi)>0\right\}\right) d \xi=0,
\end{aligned}
$$

so meas $\left(\left\{\xi \in \mathbb{R} \mid y_{\xi}(t, \xi)=0\right.\right.$ and $\left.\left.\bar{h}(t-0, \xi)>0\right\}\right)=0$ for almost every time. It follows that $\mathcal{A}$ has full measure, which concludes the proof of (202). 

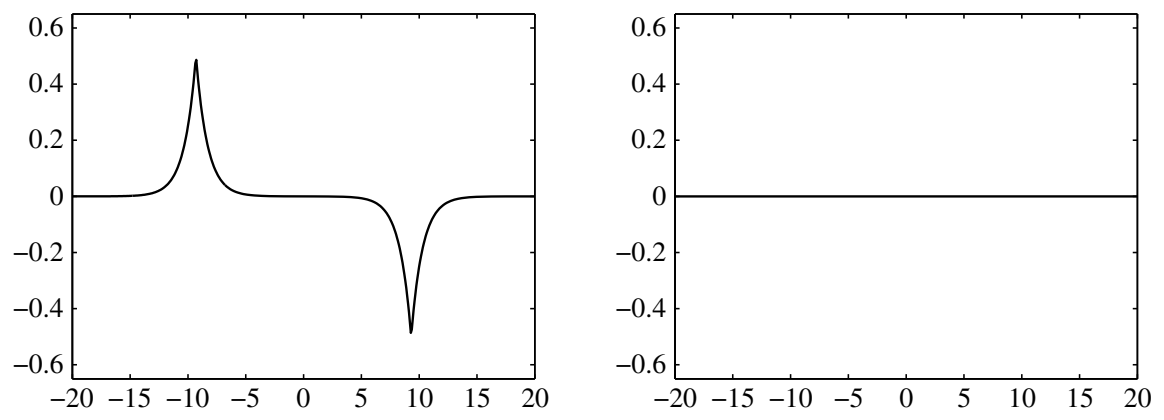

Figure 1. The peakon-antipeakon solution at times 0 and $t_{0}$ for three different values of $\alpha$. (The curves coincide.)
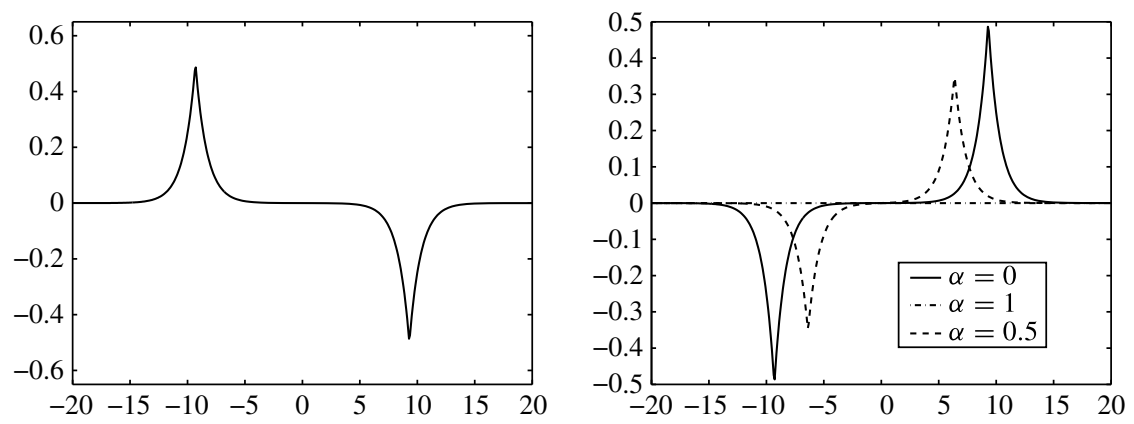

Figure 2. The peakon-antipeakon solution at times 0 and $t>t_{0}$ for three different values of $\alpha$.

\section{The peakon-antipeakon example}

The best-known explicit solution, and a key example for the dichotomy between conservative and dissipative solutions, as well as a source for intuition in the general case, is the peakon-antipeakon solution. See Figures 1 and 2. Here, we present a detailed analysis applied to this example. See, for example, $[3,26,28,36]$.

Consider the initial data

$$
u(0, x)= \begin{cases}\operatorname{sgn}(x) A(0) e^{-|x|} & \text { for }|x|>\gamma(0), \\ B(0) \sinh x & \text { for }|x| \leqslant \gamma(0)\end{cases}
$$


where we have introduced

$$
\begin{gathered}
A(t)=\frac{E}{2} \sinh \left(\frac{E}{2}\left(t-t_{0}\right)\right), \quad B(t)=E \sinh ^{-1}\left(\frac{E}{2}\left(t-t_{0}\right)\right), \\
\gamma(t)=\ln \cosh \left(\frac{E}{2}\left(t-t_{0}\right)\right),
\end{gathered}
$$

where, $\sinh ^{-1}$ denotes the multiplicative inverse of sinh. Similar conventions apply to $\cosh ^{-1}$ and $\tanh ^{-1}$. Here, $t_{0}>0$ is a given time where the wave breaking will occur. For $t<t_{0}$, the function

$$
u(t, x)= \begin{cases}\operatorname{sgn}(x) A(t) e^{-|x|} & \text { for }|x|>\gamma(t), \\ B(t) \sinh x & \text { for }|x| \leqslant \gamma(t),\end{cases}
$$

will be the peakon-antipeakon solution of the Camassa-Holm equation (1) with $\kappa=0$ and $\rho$ identically zero; see [26, Theorem 4.1, Example 4.2(ii)]. Define the two Radon measures by

$$
\mu(t)=v(t)=u_{x}^{2}(t, x) d x, \quad u_{x}(t, x)= \begin{cases}-A(t) e^{-|x|} & \text { for }|x|>\gamma(t), \\ B(t) \cosh x & \text { for }|x| \leqslant \gamma(t) .\end{cases}
$$

Observe that

$$
\int_{\mathbb{R}}\left(u^{2}(t, x)+u_{x}^{2}(t, x)\right) d x=E^{2} \quad \text { for all } t<t_{0} .
$$

At $t=t_{0}$, we see that $A\left(t_{0}\right)=\gamma\left(t_{0}\right)=0$, and thus $u\left(t_{0}, x\right)=u_{x}\left(t_{0}, x\right)=0$ almost everywhere, while $\mu(t), v(t) \rightarrow E^{2} \delta_{0}$ as $t \uparrow t_{0}$. Indeed, let $M \subset \mathbb{R}$ be a measurable set. Then

$$
\mu(t)(M)=\int_{M} u_{x}^{2}(t, x) d x \underset{t \uparrow t_{0}}{\rightarrow} \begin{cases}E^{2} & \text { for } 0 \in M, \\ 0 & \text { for } 0 \notin M,\end{cases}
$$

since $\gamma(t) \rightarrow 0$ and $u_{x}(t, x) \rightarrow 0(x \neq 0)$ as $t \uparrow t_{0}$, and

$$
\begin{aligned}
\int_{-\gamma(t)}^{\gamma(t)} u_{x}^{2}(t, x) d x & =B^{2}(t) \int_{-\gamma(t)}^{\gamma(t)} \cosh ^{2}(x) d x \\
& =B^{2}(t)\left(\gamma(t)+\frac{1}{2} \sinh (2 \gamma(t))\right) \underset{t \uparrow t_{0}}{\rightarrow} E^{2} .
\end{aligned}
$$

Next, we turn to the Lagrangian variables, which are solutions of the following system of ordinary differential equations (see (32)) for $t<t_{0}$,

$$
\begin{gathered}
y_{t}=U, \\
U_{t}=-Q,
\end{gathered}
$$




$$
\begin{gathered}
y_{t, \xi}=U_{\xi}, \\
U_{t, \xi}=\frac{1}{2} h+\left(U^{2}-P\right) y_{\xi}, \\
h_{t}=2\left(U^{2}-P\right) U_{\xi}, \\
\bar{h}_{t}=h_{t},
\end{gathered}
$$

where $P$ and $Q$ are given by (33) and (34), respectively. However, this system is difficult to solve directly, even in the case of a peakon-antipeakon solution. The initial data have to be judiciously chosen, and we will return to this shortly. Instead of solving (219) directly, we will determine the solution by using the connection between Eulerian and Lagrangian variables directly. The key relations are

$$
y_{t}=u \circ y, \quad U=u \circ y, \quad h=u_{x}^{2} \circ y y_{\xi} .
$$

We have to determine the characteristics initially (here denoted by $\bar{y}_{0}$ ), given by $(176 a)$; that is, $\bar{y}_{0}(\xi)=\sup \{y \mid v((-\infty, y))+y<\xi\}$ (we write $\bar{y}_{0}$ rather than $y_{0}$, as we will modify it shortly). In this case, where the measure $v$ is absolutely continuous, we find that the characteristics are given by

$$
\int_{-\infty}^{\bar{y}_{0}(\xi)} u_{x}^{2}(0, x) d x+\bar{y}_{0}(\xi)=\xi,
$$

which appears to be difficult to solve, even in this case. Fortunately, its derivative is straightforward:

$$
\begin{aligned}
\bar{y}_{0}^{\prime}(\xi) & =\frac{1}{1+u_{x}^{2}\left(0, \bar{y}_{0}(\xi)\right)} \\
& = \begin{cases}\left(1+A^{2}(0) e^{-\operatorname{sgn}(\xi) 2 \bar{y}_{0}(\xi)}\right)^{-1} & \text { for } \xi \notin\left[\xi_{-}, \xi_{+}\right], \\
\left(1+B^{2}(0) \cosh ^{2}\left(\bar{y}_{0}(\xi)\right)\right)^{-1} & \text { for } \xi \in\left[\xi_{-}, \xi_{+}\right],\end{cases}
\end{aligned}
$$

where we introduced $\xi_{ \pm}$, the solution of $\bar{y}_{0}\left(\xi_{ \pm}\right)= \pm \gamma(0)$. For reasons that will become clear later, we will benefit from having characteristics that satisfy $y_{0}( \pm \gamma(0))= \pm \gamma(0)$, which is not automatically satisfied by (221). We use the freedom given to us by relabeling to modify $\bar{y}_{0}$. To that end, define

$$
f(z)=\int_{-\infty}^{z} u_{x}^{2}(0, x) d x+z
$$

Then $f$ is a relabeling function in the sense of Definition 5. Observe that, with this definition, $\xi_{ \pm}=f( \pm \gamma(0))$ and $f^{\prime}(z)=u_{x}^{2}(0, z)+1$. Introduce

$$
y_{0}(z)=\bar{y}_{0}(f(z)),
$$


which implies that

$$
y_{0}( \pm \gamma(0))=\bar{y}_{0}(f( \pm \gamma(0)))=\bar{y}_{0}\left(\xi_{ \pm}\right)= \pm \gamma(0) .
$$

Hence

$$
y_{0}^{\prime}(\xi)=\bar{y}_{0}^{\prime} \circ f(\xi) f^{\prime}(\xi)=1 .
$$

Thus the relabeled initial characteristics are simply $y_{0}(\xi)=\xi$. Clearly, we could have chosen this function immediately, and the above argument shows that one can always use the identity as the initial characteristics when the initial data contains no singular part. However, the above argument illustrates the possible use of relabeling.

The Lagrangian variables are then given, using (220) for $t<t_{0}$, by

$$
\begin{aligned}
& y(t, \xi)=\left\{\begin{array}{c}
\xi+\operatorname{sgn}(\xi) \ln \left(1+\left(\cosh \left(\frac{E}{2}\left(t-t_{0}\right)\right)-\cosh \left(\frac{E}{2} t_{0}\right)\right) e^{-|\xi|}\right) \\
\quad \operatorname{for}|\xi| \geqslant \gamma(0), \\
2 \operatorname{artanh}\left(\tanh \left(\frac{\xi}{2}\right) \frac{\tanh ^{2}\left(E / 4\left(t-t_{0}\right)\right)}{\tanh ^{2}\left(-(E / 4) t_{0}\right)}\right) \text { for }|\xi| \leqslant \gamma(0),
\end{array}\right. \\
& U(t, \xi)=\left\{\begin{array}{l}
\operatorname{sgn}(\xi) A(t) e^{-|\xi|} \\
\quad \times\left(1+\left(\cosh \left(\frac{E}{2}\left(t-t_{0}\right)\right)-\cosh \left(\frac{E}{2} t_{0}\right)\right) e^{-|\xi|}\right)^{-1} \\
\operatorname{for}|\xi| \geqslant \gamma(0), \\
2 B(t) \tanh \left(\frac{\xi}{2}\right) \frac{\tanh ^{2}\left(E / 4\left(t-t_{0}\right)\right)}{\tanh ^{2}\left(-E / 4 t_{0}\right)} \\
\quad \times\left(1-\tanh ^{2}\left(\frac{\xi}{2}\right) \frac{\tanh ^{4}\left(E / 4\left(t-t_{0}\right)\right)}{\tanh ^{4}\left(-E / 4 t_{0}\right)}\right)^{-1} \text { for }|\xi| \leqslant \gamma(0),
\end{array}\right. \\
& \left\{\begin{array}{l}
A(t)^{2} e^{-2|\xi|} \\
\times\left(1+\left(\cosh \left(\frac{E}{2}\left(t-t_{0}\right)\right)-\cosh \left(\frac{E}{2} t_{0}\right)\right) e^{-|\xi|}\right)^{-3}
\end{array}\right. \\
& \text { for }|\xi| \geqslant \gamma(0) \text {, } \\
& h(t, \xi)=\left\{\begin{array}{l}
B(t)^{2}\left(1+\tanh ^{2}\left(\frac{\xi}{2}\right) \frac{\tanh ^{4}\left(E / 4\left(t-t_{0}\right)\right)}{\tanh ^{4}\left(-(E / 4) t_{0}\right)}\right)^{2}
\end{array}\right. \\
& \times\left(1-\tanh ^{2}\left(\frac{\xi}{2}\right) \frac{\tanh ^{4}\left(E / 4\left(t-t_{0}\right)\right)}{\tanh ^{4}\left(-(E / 4) t_{0}\right)}\right)^{-3} \\
& \times \cosh ^{-2}\left(\frac{\xi}{2}\right) \frac{\tanh ^{2}\left(E / 4\left(t-t_{0}\right)\right)}{\tanh ^{2}\left(-(E / 4) t_{0}\right)} \text { for }|\xi| \leqslant \gamma(0),
\end{aligned}
$$




$$
\begin{aligned}
& \bar{h}(t, \xi)=h(t, \xi), \\
& P(t, \xi)=\frac{1}{4} \int_{\mathbb{R}} e^{-|y(t, \xi)-y(t, \eta)|}\left(2 U^{2} y_{\xi}+h\right)(t, \eta) d \eta, \\
& Q(t, \xi)=-\frac{1}{4} \int_{\mathbb{R}} \operatorname{sgn}(\xi-\eta) e^{-|y(t, \xi)-y(t, \eta)|}\left(2 U^{2} y_{\xi}+h\right)(t, \eta) d \eta .
\end{aligned}
$$

With the choice of initial characteristics, we obtain

$$
y(t, \pm \gamma(0))= \pm \gamma(t)
$$

and hence at the peaks

$$
U(t, \pm \gamma(0))=u(t, \pm \gamma(t))= \pm \frac{E}{2} \tanh \left(\frac{E}{2}\left(t-t_{0}\right)\right)
$$

As expected,

$$
\tau(\xi)= \begin{cases}\infty & \text { for }|\xi| \geqslant \gamma(0) \\ t_{0} & \text { for }|\xi|<\gamma(0)\end{cases}
$$

The important quantity is the first time there is wave breaking. By construction,

$$
t_{0}=\inf _{\xi} \tau(\xi)
$$

Next, we consider the limits of these variables as $t \uparrow t_{0}$ :

$$
\lim _{t \uparrow t_{0}} y(t, \xi)= \begin{cases}\xi+\operatorname{sgn}(\xi) \ln \left(1+\left(1-\cosh \left(\frac{E}{2}\right) t_{0}\right) e^{-|\xi|}\right) & \text { for }|\xi| \geqslant \gamma(0), \\ 0 & \text { for }|\xi|<\gamma(0),\end{cases}
$$

$\lim _{t \uparrow t_{0}} U(t, \xi)=0$,

$$
\begin{aligned}
& \lim _{t \uparrow t_{0}} h(t, \xi)= \begin{cases}0 & \text { for }|\xi| \geqslant \gamma(0), \\
\frac{E^{2}}{4} \cosh ^{-2}\left(\frac{\xi}{2}\right) \tanh ^{-2}\left(-\frac{E}{4} t_{0}\right) & \text { for }|\xi| \leqslant \gamma(0),\end{cases} \\
& \lim _{t \uparrow t_{0}} P(t, \xi)= \begin{cases}\frac{E^{2}}{4}\left(1+\left(1-\cosh \left(\frac{E}{2} t_{0}\right)\right) e^{-|\xi|}\right)^{-1} e^{-|\xi|} & \text { for }|\xi| \geqslant \gamma(0), \\
\frac{E^{2}}{4} & \text { for }|\xi| \leqslant \gamma(0),\end{cases}
\end{aligned}
$$


$\lim _{t \uparrow t_{0}} Q(t, \xi)=-\left\{\begin{array}{l}\operatorname{sgn}(\xi) \frac{E^{2}}{4}\left(1+\left(1-\cosh \left(\frac{E}{2} t_{0}\right)\right) e^{-|\xi|}\right)^{-1} e^{-|\xi|} \\ \text { for }|\xi| \geqslant \gamma(0), \\ \frac{E^{2}}{4} \tanh ^{-2}\left(-\frac{E}{4} t_{0}\right) \tanh \left(\frac{\xi}{2}\right) \\ \text { for }|\xi| \leqslant \gamma(0) .\end{array}\right.$

At $t=t_{0}$, we introduce the parameter $\alpha \in[0,1]$, and define

$$
\bar{h}\left(t_{0}, \xi\right)=(1-\alpha) \lim _{t \uparrow t_{0}} h(t, \xi), \quad h\left(t_{0}, \xi\right)=\lim _{t \uparrow t_{0}} h(t, \xi) .
$$

This implies that, in Eulerian variables,

$$
u\left(t_{0}, x\right)=0, \quad \mu\left(t_{0}\right)=(1-\alpha) E^{2} \delta_{0}, \quad \text { and } \quad v\left(t_{0}\right)=E^{2} \delta_{0},
$$

using Definitions (191b) and (191c), namely, $\mu=y_{\#}(\bar{h}(\xi) d \xi)$ and $v=$ $y_{\#}(h(\xi) d \xi)$.

We will show that for $t>t_{0}$ the solution coincides with the peakon-antipeakon solution with the energy $E$ replaced by

$$
\tilde{E}=\sqrt{1-\alpha} E
$$

For $t>t_{0}$, the Lagrangian system reads (see (32))

$$
\begin{gathered}
y_{t}=U, \\
U_{t}=-Q, \\
y_{t, \xi}=U_{\xi}, \\
U_{t, \xi}=\frac{1}{2} \bar{h}+\left(U^{2}-P\right) y_{\xi}, \\
h_{t}=2\left(U^{2}-P\right) U_{\xi}, \\
\bar{h}_{t}=h_{t},
\end{gathered}
$$

where $P$ and $Q$ are given by (33) and (34), respectively.

In the fully dissipative case with $\alpha=1$, we get $\bar{h}\left(t_{0}\right)=0$, but also $\left(U^{2}-\right.$ $P)\left(t_{0}\right)=U_{\xi}\left(t_{0}\right)=Q\left(t_{0}\right)=0$, and hence we have, for $t>t_{0}$,

$$
\begin{aligned}
y(t, \xi) & =y\left(t_{0}, \xi\right), \\
U(t, \xi) & =0, \\
h(t, \xi) & =h\left(t_{0}, \xi\right), \\
\bar{h}(t, \xi) & =0 .
\end{aligned}
$$


This implies that, in Eulerian variables,

$$
u(t, x)=0, \quad \mu(t)=0, \quad v(t)=E^{2} \delta_{0}, \quad t>t_{0} .
$$

In the general case $\alpha \in[0,1)$, it is difficult, as it was for $t<t_{0}$, to solve system (236) explicitly. However, we proceed as follows. Given (234), we use (176a), denoting the characteristics by $\tilde{y}\left(t_{0}\right)$, to determine the new initial characteristics. We find that

$$
\tilde{y}\left(t_{0}, \xi\right)= \begin{cases}\xi & \text { for } \xi \leqslant 0, \\ 0 & \text { for } 0 \leqslant \xi<\tilde{E}^{2}, \\ \xi-\tilde{E}^{2} & \text { for } \xi \geqslant \tilde{E}^{2} .\end{cases}
$$

Note that this function is related by relabeling to the characteristics we already have at $t=t_{0}$, given by (232), namely

$$
y\left(t_{0}, \xi\right)= \begin{cases}\xi+\operatorname{sgn}(\xi) \ln \left(1+\left(1-\cosh \left(\frac{E}{2} t_{0}\right)\right) e^{-|\xi|}\right) & \text { for }|\xi| \geqslant \gamma(0) \\ 0 & \text { for }|\xi|<\gamma(0)\end{cases}
$$

To that end, define

$$
\begin{aligned}
g(\xi)= & y\left(t_{0}, \xi\right)+\bar{H}\left(t_{0}, \xi\right)=y\left(t_{0}, \xi\right)+\int_{-\infty}^{\xi} \bar{h}\left(t_{0}, \eta\right) d \eta \\
= & \begin{cases}\xi-\ln \left(1+\left(1-\cosh \left(\frac{E}{2} t_{0}\right)\right) e^{\xi}\right) & \text { for } \xi \leqslant-\gamma(0), \\
\frac{\tilde{E}^{2}}{2}\left(\tanh ^{-2}\left(-\frac{E}{4} t_{0}\right) \tanh \left(\frac{\xi}{2}\right)+1\right) & \text { for }-\gamma(0) \leqslant \xi<\gamma(0), \\
\xi+\tilde{E}^{2}+\ln \left(1+\left(1-\cosh \left(\frac{E}{2} t_{0}\right)\right) e^{-\xi}\right) & \text { for } \xi \geqslant \gamma(0) .\end{cases}
\end{aligned}
$$

Observe that $g$ is a monotonically increasing relabeling function that satisfies

$$
\lim _{\xi \rightarrow-\gamma(0)} g(\xi)=0, \quad \lim _{\xi \rightarrow \gamma(0)} g(\xi)=\tilde{E}^{2},
$$

and thus

$$
y\left(t_{0}, \xi\right)=\tilde{y}\left(t_{0}, g(\xi)\right) .
$$

We are now given initial data $y\left(t_{0}\right)$, as well as $U\left(t_{0}\right)=0$ and $h\left(t_{0}\right)$. We claim that the solution, in Eulerian variables, is

$$
u(t, x)=\left\{\begin{array}{ll}
\operatorname{sgn}(x) \tilde{A}(t) e^{-|x|} & \text { for }|x|>\tilde{\gamma}(t), \\
\tilde{B}(t) \sinh x & \text { for }|x| \leqslant \tilde{\gamma}(t),
\end{array} \quad t>t_{0},\right.
$$




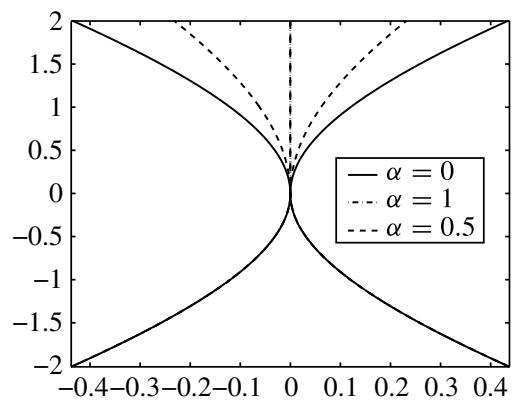

Figure 3. The characteristics $y(t, \pm \gamma(0))$ describing the location of the peaks of the peakon-antipeakon solution for three different values of $\alpha$.

where

$$
\begin{gathered}
\tilde{A}(t)=\frac{\tilde{E}}{2} \sinh \left(\frac{\tilde{E}}{2}\left(t-t_{0}\right)\right), \\
\tilde{B}(t)=\tilde{E} \sinh ^{-1}\left(\frac{\tilde{E}}{2}\left(t-t_{0}\right)\right), \quad \tilde{\gamma}(t)=\ln \cosh \left(\frac{\tilde{E}}{2}\left(t-t_{0}\right)\right) .
\end{gathered}
$$

To determine the characteristics, we solve the equation $y_{t}=u \circ y$. We provide some details. Consider first the case $\xi \leqslant-\gamma(0)$. Integrating, we find that

$$
e^{-y(t, \xi)}-e^{-y\left(t_{0}, \xi\right)}=\cosh \left(\frac{\tilde{E}}{2}\left(t-t_{0}\right)\right)-1 .
$$

Inserting the expression (240), we find that

$$
y(t, \xi)=\xi-\ln \left(1+\left(\cosh \left(\frac{\tilde{E}}{2}\left(t-t_{0}\right)\right)-\cosh \left(\frac{E}{2} t_{0}\right)\right) e^{\xi}\right) .
$$

A similar calculation determines the case $\xi \geqslant \gamma(0)$. Assume now that $-\gamma(0) \leqslant$ $\xi \leqslant \gamma(0)$. Integrating the equation, we find, for any small positive $\varepsilon$, that

$$
\begin{gathered}
\ln \tanh \left(\frac{1}{2} y(t, \xi)\right)-\ln \tanh \left(\frac{1}{2} y\left(t_{0}+\varepsilon, \xi\right)\right) \\
\quad=2\left(\ln \tanh \left(\frac{\tilde{E}}{4}\left(t-t_{0}\right)\right)-\ln \tanh \left(\frac{\tilde{E}}{4} \varepsilon\right)\right),
\end{gathered}
$$

which can be rewritten as

$$
y(t, \xi)=2 \operatorname{artanh}\left(\tanh \left(\frac{1}{2} y\left(t_{0}+\varepsilon, \xi\right)\right) \frac{\tanh ^{2}\left(\tilde{E} / 4\left(t-t_{0}\right)\right)}{\tanh ^{2}((\tilde{E} / 4) \varepsilon)}\right) .
$$


Taking $\varepsilon \downarrow 0$, we find that

$$
y(t, \xi)=2 \operatorname{artanh}\left(\tanh \left(\frac{\xi}{2}\right) \frac{\tanh ^{2}\left(\tilde{E} / 4\left(t-t_{0}\right)\right)}{\tanh ^{2}\left(-(E / 4) t_{0}\right)}\right) .
$$

Note that this limit is rather delicate. As it involves repeated use of L'Hôpital's rule, one has to invoke equations (236) in order to compute the limit. We can now determine the remaining Lagrangian quantities:

$$
\begin{aligned}
& y(t, \xi)=\left\{\begin{array}{c}
\xi+\operatorname{sgn}(\xi) \ln \left(1+\left(\cosh \left(\frac{\tilde{E}}{2}\left(t-t_{0}\right)\right)-\cosh \left(\frac{E}{2} t_{0}\right)\right) e^{-|\xi|}\right) \\
\quad \text { for }|\xi| \geqslant \gamma(0), \\
2 \operatorname{artanh}\left(\tanh \left(\frac{\xi}{2}\right) \frac{\tanh ^{2}\left(\tilde{E} / 4\left(t-t_{0}\right)\right)}{\tanh ^{2}\left(-(E / 4) t_{0}\right)}\right) \quad \text { for }|\xi| \leqslant \gamma(0),
\end{array}\right. \\
& \int \operatorname{sgn}(\xi) \tilde{A}(t) e^{-|\xi|}\left(1+\left(\cosh \left(\frac{\tilde{E}}{2}\left(t-t_{0}\right)\right)-\cosh \left(\frac{E}{2} t_{0}\right)\right) e^{-|\xi|}\right)^{-1} \\
& \text { for }|\xi| \geqslant \gamma(0) \\
& U(t, \xi)=\left\{\begin{array}{l}
2 \tilde{B}(t) \tanh \left(\frac{\xi}{2}\right) \frac{\tanh ^{2}\left(\tilde{E} / 4\left(t-t_{0}\right)\right)}{\tanh ^{2}\left(-(E / 4) t_{0}\right)}
\end{array}\right. \\
& \times\left(1-\tanh ^{2}\left(\frac{\xi}{2}\right) \frac{\tanh ^{4}\left(\tilde{E} / 4\left(t-t_{0}\right)\right)}{\tanh ^{4}\left(-(E / 4) t_{0}\right)}\right)^{-1} \text { for }|\xi| \leqslant \gamma(0), \\
& \tilde{A}(t)^{2} e^{-2|\xi|}\left(1+\left(\cosh \left(\frac{\tilde{E}}{2}\left(t-t_{0}\right)\right)-\cosh \left(\frac{E}{2} t_{0}\right)\right) e^{-|\xi|}\right)^{-3} \\
& \text { for }|\xi| \geqslant \gamma(0) \text {, } \\
& \bar{h}(t, \xi)=\left\{\begin{array}{c}
\tilde{B}(t)^{2}\left(1+\tanh ^{2}\left(\frac{\xi}{2}\right) \frac{\tanh ^{4}\left(\tilde{E} / 4\left(t-t_{0}\right)\right)}{\tanh ^{4}\left(-(E / 4) t_{0}\right)}\right)^{2} \\
\times\left(1-\tanh ^{2}\left(\frac{\xi}{2}\right) \frac{\tanh ^{4}\left(\tilde{E} / 4\left(t-t_{0}\right)\right)}{\tanh ^{4}\left(-(E / 4) t_{0}\right)}\right)^{-3}
\end{array}\right. \\
& \times \cosh ^{-2}\left(\frac{\xi}{2}\right) \frac{\tanh ^{2}\left(\tilde{E} / 4\left(t-t_{0}\right)\right)}{\tanh ^{2}\left(-(E / 4) t_{0}\right)} \\
& \text { for }|\xi| \leqslant \gamma(0) \text {, } \\
& h(t, \xi)= \begin{cases}\bar{h}(t, \xi) & \text { for }|\xi| \geqslant \gamma(0), \\
\bar{h}(t, \xi)+\alpha \frac{E^{2}}{4} \cosh ^{-2}\left(\frac{\xi}{2}\right) \tanh ^{-2}\left(-\frac{E}{4} t_{0}\right) & \text { for }|\xi|<\gamma(0),\end{cases}
\end{aligned}
$$




$$
\begin{aligned}
& P(t, \xi)=\frac{1}{4} \int_{\mathbb{R}} e^{-|y(t, \xi)-y(t, \eta)|}\left(2 U^{2} y_{\xi}+\bar{h}\right)(t, \eta) d \eta, \\
& Q(t, \xi)=-\frac{1}{4} \int_{\mathbb{R}} \operatorname{sgn}(\xi-\eta) e^{-|y(t, \xi)-y(t, \eta)|}\left(2 U^{2} y_{\xi}+\bar{h}\right)(t, \eta) d \eta .
\end{aligned}
$$

To complete the calculation of the Eulerian variables, we use Definitions (191b) and $(191 c)$ to determine the measures. First, we find that

$$
\mu(t)=u_{x}^{2}(t, x) d x, \quad t>t_{0} .
$$

To determine $v(t)$, we write $h=\bar{h}+l_{1}$ (see (31)) with $\bar{h}=u_{x}^{2} \circ y y_{\xi}$. We want to determine a function $l$ such that $l_{1}=l \circ y y_{\xi}$, which implies that $v=(\bar{h}+l) d x$. From (251), we see that $l_{1}(t, \xi)=0$ for $|\xi| \geqslant \gamma(0)$. For $|\xi|<\gamma(0)$, we first observe from (251) that

$$
\begin{aligned}
y_{\xi}(t, \xi)= & \left(1-\left(\tanh \left(\frac{\xi}{2}\right) \frac{\tanh ^{2}\left(\tilde{E} / 4\left(t-t_{0}\right)\right)}{\tanh ^{2}\left(-(E / 4) t_{0}\right)}\right)^{2}\right)^{-1} \\
& \times \cosh ^{-2}\left(\frac{\xi}{2}\right) \frac{\tanh ^{2}\left(\tilde{E} / 4\left(t-t_{0}\right)\right)}{\tanh ^{2}\left(-(E / 4) t_{0}\right)} \\
= & \left(1-\tanh ^{2}\left(\frac{y(t, \xi)}{2}\right)\right)^{-1} \cosh ^{-2}\left(\frac{\xi}{2}\right) \frac{\tanh ^{2}\left(\tilde{E} / 4\left(t-t_{0}\right)\right)}{\tanh ^{2}\left(-(E / 4) t_{0}\right)}
\end{aligned}
$$

using

$$
\tanh \left(\frac{y(t, \xi)}{2}\right)=\tanh \left(\frac{\xi}{2}\right) \frac{\tanh ^{2}\left(\tilde{E} / 4\left(t-t_{0}\right)\right)}{\tanh ^{2}\left(-(E / 4) t_{0}\right)} .
$$

Thus, for $|\xi|<\gamma(0)$,

$$
\begin{aligned}
l(t, y(t, \xi)) & =\frac{l_{1}(t, \xi)}{y_{\xi}(t, \xi)} \\
& =\alpha \frac{E^{2}}{4} \frac{\left(1-\tanh ^{2}(y(t, \xi) / 2)\right) \cosh ^{2}(\xi / 2) \tanh ^{2}\left(-(E / 4) t_{0}\right)}{\cosh ^{2}(\xi / 2) \tanh ^{2}\left(-(E / 4) t_{0}\right) \tanh ^{2}\left(\tilde{E} / 4\left(t-t_{0}\right)\right)} \\
& =\alpha \frac{E^{2}}{4}\left(1-\tanh ^{2}\left(\frac{y(t, \xi)}{2}\right)\right) \tanh ^{-2}\left(\tilde{E} / 4\left(t-t_{0}\right)\right),
\end{aligned}
$$

and thus we infer that

$$
l(t, x)= \begin{cases}0 & \text { for }|x| \geqslant \tilde{\gamma}(t), \\ \alpha \frac{E^{2}}{4}\left(1-\tanh ^{2}\left(\frac{x}{2}\right)\right) \tanh ^{-2}\left(\frac{\tilde{E}}{4}\left(t-t_{0}\right)\right) & \text { for }|x| \leqslant \tilde{\gamma}(t) .\end{cases}
$$


Finally, we get the following expression:

$$
v(t)=\left\{\begin{array}{l}
u_{x}^{2}(t, x) d x \quad \text { for }|x| \geqslant \tilde{\gamma}(t), \\
\left(u_{x}^{2}(t, x)+\alpha \frac{E^{2}}{4} \tanh ^{-2}\left(\frac{\tilde{E}}{4}\left(t-t_{0}\right)\right)\left(1-\tanh ^{2}\left(\frac{x}{2}\right)\right)\right) d x \\
\quad \text { for }|x| \leqslant \tilde{\gamma}(t),
\end{array}\right.
$$

for $t>t_{0}$.

\section{Acknowledgements}

This research was supported in part by the Research Council of Norway and by the Austrian Science Fund (FWF) under Grant No. J3147.

\section{References}

[1] L. Ambrosio, N. Fusco and D. Pallara, Functions of Bounded Variation and Free Discontinuity Problems (Clarendon Press, New York, 2000).

[2] H. Aratyn, J. F. Gomes and A. H. Zimerman, 'On a negative flow of the AKNS hierarchy and its relation to a two-component Camassa-Holm equation', in Symmetry, Integrability and Geometry: Methods and Applications, 2 (2006), Paper 070, 12 pages.

[3] R. Beals, D. Sattinger and J. Szmigielski, 'Peakon-antipeakon interaction', J. Nonlinear Math. Phys. 8 (2001), 23-27.

[4] A. Bressan and A. Constantin, 'Global conservative solutions of the Camassa-Holm equation', Arch. Ration. Mech. Anal. 183 (2007), 215-239.

[5] A. Bressan and A. Constantin, 'Global dissipative solutions of the Camassa-Holm equation', Anal. Appl. 5 (2007), 1-27.

[6] A. Bressan, H. Holden and X. Raynaud, 'Lipschitz metric for the Hunter-Saxton equation', J. Math. Pures Appl. 94 (2010), 68-92.

[7] R. Camassa and D. D. Holm, 'An integrable shallow water equation with peaked solitons', Phys. Rev. Lett. 71(11) (1993), 1661-1664.

[8] M. Chen, S.-Q. Liu and Y. Zhang, 'A two-component generalization of the Camassa-Holm equation and its solutions', Lett. Math. Phys. 75 (2006), 1-15.

[9] R. M. Chen and Y. Liu, Wave breaking and global existence for a generalized two-component Camassa-Holm system. Inter. Math Research Notices, Article ID rnq118, 36 pages, 2010.

[10] A. Constantin and J. Escher, 'Wave breaking for nonlinear nonlocal shallow water equations', Acta Math. 181 (1998), 229-243.

[11] A. Constantin and R. I. Ivanov, 'On an integrable two-component Camassa-Holm shallow water system', Phys. Lett. A 372 (2008), 7129-7132.

[12] J. Escher, O. Lechtenfeld and Z. Yin, 'Well-posedness and blow-up phenomena for the 2-component Camassa-Holm equation', Discrete Contin. Dyn. Syst. 19(3) (2007), 493-513.

[13] Y. Fu and C. Qu, 'Well posedness and blow-up solution for a new coupled Camassa-Holm equations with peakons', J. Math. Phys. 50012906 (2009).

[14] K. Grunert, H. Holden and X. Raynaud, 'Global conservative solutions of the CamassaHolm equation for initial data with nonvanishing asymptotics', Discrete Cont. Dyn. Syst. A 32 (2012), 4209-4277. 
[15] K. Grunert, H. Holden and X. Raynaud, 'Global solutions for the two-component CamassaHolm system', Comm. Partial Differential Equations 37 (2012), 2245-2271.

[16] K. Grunert, H. Holden and X. Raynaud, 'Lipschitz metric for the Camassa-Holm equation on the line', Discrete Contin. Dyn. Syst. 33 (2013), 2809-2827.

[17] K. Grunert, H. Holden and X. Raynaud, 'Periodic conservative solutions for the twocomponent Camassa-Holm system', in Spectral Analysis, Differential Equations and Mathematical Physics (eds. H. Holden, B. Simon and G. Teschl), (American Mathematical Society, 2013), 165-182. A Festschrift for Fritz Gesztesy on the Occasion of his 60th Birthday.

[18] K. Grunert, H. Holden and X. Raynaud, 'Global dissipative solutions of the two-component Camassa-Holm system for initial data with nonvanishing asymptotics', Nonlinear Anal. Real World Appl. 17 (2014), 203-244.

[19] C. Guan, K. H. Karlsen and Z. Yin, 'Well-posedness and blow-up phenomenal for a modified two-component Camassa-Holm equation', in Nonlinear Partial Differential Equations and Hyperbolic Wave Phenomena (eds. H. Holden and K. H. Karlsen), Contemporary Mathematics, 526 (American Mathematical Society, 2010), 199-220.

[20] C. Guan and Z. Yin, 'Global existence and blow-up phenomena for an integrable twocomponent Camassa-Holm water system', J. Differential Equations 248 (2010), 2003-2014.

[21] C. Guan and Z. Yin, 'Global weak solutions for a modified two-component Camassa-Holm equation’, Ann. Inst. H. Poincaré Anal. Non Linéaire 28 (2011), 623-641.

[22] C. Guan and Z. Yin, 'Global weak solutions for a two-component Camassa-Holm shallow water system', J. Funct. Anal. 260 (2011), 1132-1154.

[23] G. Gui and Y. Liu, 'On the Cauchy problem for the two-component Camassa-Holm system', Math. Z. 268 (2011), 45-66.

[24] G. Gui and Y. Liu, 'On the global existence and wave breaking criteria for the twocomponent Camassa-Holm system', J. Funct. Anal. 258 (2010), 4251-4278.

[25] Z. Guo and Y. Zhou, 'On solutions to a two-component generalized Camassa-Holm equation', Stud. Appl. Math. 124 (2010), 307-322.

[26] H. Holden and X. Raynaud, 'Global conservative multipeakon solutions of the CamassaHolm equation', J. Hyperbolic Differ. Equ. 4 (2007), 39-64.

[27] H. Holden and X. Raynaud, 'Global conservative solutions of the Camassa-Holm equation - a Lagrangian point of view', Comm. Partial Differential Equations 32 (2007), 1511-1549.

[28] H. Holden and X. Raynaud, 'Global dissipative multipeakon solutions for the CamassaHolm equation', Comm. Partial Differential Equations 33 (2008), 2040-2063.

[29] H. Holden and X. Raynaud, 'Dissipative solutions of the Camassa-Holm equation', Discrete Contin. Dyn. Syst. 24 (2009), 1047-1112.

[30] D. D. Holm, L. Ó. Náraigh and C. Tronci, 'Singular solutions of a modified two-component Camassa-Holm equation', Phys. Rev. E 79016601 (2009).

[31] Q. Hu and Z. Yin, 'Well-posedness and blow-up phenomena for a periodic two-component Camassa-Holm equation', Proc. Roy. Soc. Edinburgh 141A (2011), 93-107.

[32] R. I. Ivanov, 'Extended Camassa-Holm hierarchy and conserved quantities', Z. Natforsch. 61A (2006), 133-138.

[33] P. A. Kuz'min, 'Two-component generalizations of the Camassa-Holm equation', Math. Notes 81 (2007), 130-134.

[34] L. Tian, Y. Wang and J. Zhou, 'Global conservative and dissipative solutions of a coupled Camassa-Holm equations', J. Math. Phys. 52063702 (2011). 
[35] P. J. Olver and P. Rosenau, 'Tri-hamiltonian duality between solitons and solitary-wave solutions having compact support', Phys. Rev. B 53(2) (1996), 1900-1906.

[36] E. Wahlén, 'The interaction of peakons and antipeakons', Dyn. Contin. Discrete Impuls. Syst. A 13 (2006), 465-472.

[37] Y. Wang, J. Huang and L. Chen, 'Global conservative solutions of the two-component Camassa-Holm shallow water system’, Int. J. Nonlinear Sci. 9 (2009), 379-384. 